Atratores globais para uma equação viscoelástica não linear com história

Paulo Nicanor Seminario Huertas 

SERVIÇO DE PÓS-GRADUAÇÃO DO ICMC-USP

Data de Depósito:

Assinatura:

\title{
Atratores globais para uma equação viscoelástica não linear com história
}

\author{
Paulo Nicanor Seminario Huertas
}

Orientador: Prof. Dr. Ma To Fu

Dissertação apresentada ao Instituto de Ciências Matemáticas e de Computação - ICMC-USP, como parte dos requisitos para obtenção do título de Mestre em Ciências - Ciências de Computação e Matemática Computacional. EXEMPLAR DE DEFESA.

USP - São Carlos

Janeiro de 2015 
Ficha catalográfica elaborada pela Biblioteca Prof. Achille Bassi e Seção Técnica de Informática, ICMC/USP, com os dados fornecidos pelo(a) autor(a)

Huertas, Paulo Nicanor Seminario

H887a Atratores globais para uma equação viscoelástica não linear com história / Paulo Nicanor Seminario Huertas; orientador Ma To Fu. -- São Carlos, 2015. $74 \mathrm{p}$.

Dissertação (Mestrado - Programa de Pós-Graduação em Matemática) -- Instituto de Ciências Matemáticas e de Computação, Universidade de São Paulo, 2015.

1. Equações diferenciais parciais. 2. Equações da onda. 3. Atratores globais. 4. Equação viscoelástica com história. I. Fu, Ma To, orient. II. Título. 
Aunque me fuercen, yo nunca voy a decir que todo tiempo por pasado fue mejor. Mañana es mejor. - "Cantata de puentes amarillos". L. A. Spinetta 



\section{Agradecimentos}

Primeiramente quero agradecer em especial a meu orientador o Prof. Dr. Ma To Fu, por sua dedicação, paciência, ajuda, amizade, apoio em todo o tempo que ele me orientou.

A meus queridos pais, Mercedes Elena Huertas Porras e Oswaldo Angel Robles Rodriguez que sempre me apoiaram, me deram sua compreensão, amor e pelo sacrifício que fizeram para continuar com meus estudos e chegar até onde estou. A meu pai de sangue, Paulo N. Seminario Bohorquez que me apoiou e o qual agradeço a vida. A minha irmã Mercedes E. Seminario Huertas assim como a meus avós Antonieta Porras e Aurelio Huertas pela amizade e pelo apoio de forma incondicional durante toda minha vida.

Aos professores do departamento de Matemática do ICMC que contribuíram com a minha formação pelos ensinamentos, apoio e motivação.

Aos funcionários da Biblioteca do ICMC, das seções de pós-graduação, da área financeira e da diretoria.

Aos meus amigos Alfredo Sotelo, Miguel Angel Zambrano, Karol Veliz, Dante Baldeon, Patricia Hilario, Norbil Cordova, Lito Bocanegra, Felipe Correa, Yonny, etc... todos eles tem minha gratidão por todo o apoio.

A Spinetta, Charly e Fito que estão sempre comigo em todo momento.

À CAPES e ao CNPq pelo apoio financeiro sem o qual não teria sido possível este trabalho.

Peço desculpas às pessoas que não citei e que me ajudaram direta ou indireitamente no mestrado, agradeço muito. 


\section{Resumo}

Neste trabalho estudamos uma classe de equações de ondas da forma

$$
\left|\partial_{t} u\right|^{\rho} \partial_{t t} u-\Delta \partial_{t t} u-\alpha \Delta u+\int_{0}^{\infty} \mu(s) \Delta u(t-s) d s+f(u)=h
$$

definida num domínio limitado de $\mathbb{R}^{3}$, com condição de fronteira de Dirichlet e parâmetros $\alpha, \rho>0$.

Tais equações modelam problemas de viscoelasticidade não linear e têm sido estudados por diversos autores. Aqui, apresentamos um teorema de existência, unicidade e dependência contínua em relação aos dados iniciais, para soluções fracas, como discutido por Conti, Marchini \& Pata (2014).

Em seguida provamos um teorema novo sobre a existência de atratores globais para o sistema dinâmico associado ao problema, explorando tão somente a dissipação dada pelo termo de memória. Tal resultado generaliza substancialmente o trabalho pioneiro de Araújo, Ma \& Qin (2013). 


\section{Abstract}

In this work we study a class of wave equations of the form

$$
\left|\partial_{t} u\right|^{\rho} \partial_{t t} u-\Delta \partial_{t t} u-\alpha \Delta u+\int_{0}^{\infty} \mu(s) \Delta u(t-s) d s+f(u)=h
$$

defined in a bounded domain of $\mathbb{R}^{3}$, with Dirichlet boundary condition and parameters $\alpha, \rho>0$.

Such equations model problems from nonlinear visco-elasticity and have been considered by several authors. Here, we prove the well-posedness of the problem, as discussed by Conti, Marchini \& Pata (2014).

Next, we prove a new result on the existence of global attractors for the dynamical system generated by the problem, by exploring the dissipation the memory term only. The result extends substantially the pioneering work by Araújo, Ma \& Qin (2013). 


\section{Sumário}

Introdução

1 Preliminares 5

1.1 Espaços de Sobolev . . . . . . . . . . . . . . . . . . 5 5

1.2 Espaços de Bochner com peso . . . . . . . . . . . . . . . . 11

1.3 Sistemas dinâmicos e atratores globais . . . . . . . . . . . . . 12

1.4 Soluções fracas (mild solutions) $\ldots \ldots \ldots \ldots$. . . . . . . . . . 15

2 Equação viscoelástico não-linear com história $\quad 17$

2.1 Entorno funcional . . . . . . . . . . . . . . . . . . . . . 18

2.2 Problema com história . . . . . . . . . . . . . . . . . 21

3 Boa colocação do problema $\quad 25$

3.1 Existência de soluções fracas . . . . . . . . . . . . . . . . 28

3.1 .1 Problema aproximado . . . . . . . . . . . . . 29

3.1 .2 Primeira estimativa . . . . . . . . . . . . 30

3.1 .3 Segunda estimativa . . . . . . . . . . . . . 35

3.1 .4 Terceira estimativa . . . . . . . . . . . . . 36

3.1.5 Passagem ao limite . . . . . . . . . . . . . . 37

3.2 Dependência contínua e unicidade das soluções fracas . . . . . . . . . . . 39

3.2.1 Dependência contínua das soluções fracas . . . . . . . . . . . 39

3.2 .2 Unicidade das soluções fracas . . . . . . . . . . . . . . 48

4 Existência de atrator global $\quad 49$

4.1 Dissipatividade ............................. 51

4.2 Desigualdade de estabilização . . . . . . . . . . . . . . 56

4.3 Compacidade assintótica . . . . . . . . . . . . . . . 68 
Referências Bibliográficas 


\section{Introdução}

Nessa dissertação estudamos a dinâmica assintótica de uma equação de ondas não linear da forma

$$
\left|\partial_{t} u\right|^{\rho} \partial_{t t} u-\Delta \partial_{t t} u-\alpha \Delta u+\int_{0}^{\infty} \mu(s) \Delta u(t-s) d s+f(u)=h,
$$

definida numa região limitada $\Omega \subset \mathbb{R}^{3}$, adicionados de dados iniciais e condição de fronteira do tipo Dirichlet.

Essa classe de equações foi primeiramente estudada por Cavalcanti, Domingos Cavalcanti \& Ferreira [6], como uma versão visco-elástica do problema de vibrações

$$
g\left(u_{t}\right) u_{t t}-\Delta u-\Delta u_{t t}=0
$$

onde $g\left(u_{t}\right)$ representa uma forma não linear de densidade de material. No caso $g\left(u_{t}\right)=1$, essa equação modela vibrações de hastes finas conforme justificado em Love [22, Capítulo 20]. O caráter visco-elástico da equação (1) vem da integral de convolução que modela efeitos de memória do material, cf. por exemplo, Fabrizio \& Morro [13].

Em Cavalcanti, Domingos Cavalcanti \& Ferreira [6] o problema (1) foi estudado na forma

$$
\left|\partial_{t} u\right|^{\rho} \partial_{t t} u-\Delta \partial_{t t} u-\alpha \Delta u+\int_{0}^{t} \mu(t-s) \Delta u(s) d s=\mathcal{F} .
$$

Supondo-se $\rho \in(0,2]$ e que $\mu(t)$ decai exponencialmente a zero quando $t \rightarrow \infty$, eles provaram que a energia do sistema decai exponencialmente quando acrescentamos uma dissipação forte $\mathcal{F}=\Delta u_{t}$. A partir daquele trabalho muitos outros autores aportaram novas contribuições para equações do tipo (2), por exemplo, [17, 18, 20, 21, 25, 26, 27, 37, 38]. Entre esses, destacamos o trabalho de Messaoudi \& Tatar [26] onde foi provado que o decaimento exponencial da energia continua válido com $\mathcal{F}=0$, explorando somente a dissipação dada pelo termo de memória. 
Observamos que se $\rho=0$ (densidade constante) e $\Delta u_{t t}$ for removida, então a equação (1) se reduz a bem conhecida equação de onda visco-elástica. Nessa direção o problema foi estudada por diferentes autores, por exemplo, em [7, 8, 24, 29, 30, 35], entre outros.

Por outro lado, em todos trabalhos acima citados sobre o problema (2), a questão da unicidade de soluções nunca é tratada. De fato, há uma aparente dificuldade devida ao termo $\left|u_{t}\right|^{\rho} u_{t t}$. A primeira prova da boa colocação do problema, e portanto sua unicidade, foi estabelecida recentemente em Araújo, Ma \& Qin [2], através da restrição $\rho>1$. O resultado foi apresentado num contexto mais geral envolvendo memória com história como na equação (1). Aqui, o conceito de história vem do fato que no termo de convolução na equação (1) precisamos conhecer os valores de $u(x, t) \operatorname{com} t \in(-\infty, t]$. Mais tarde, Conti, Marchini \& Pata [11] apresentaram um novo resultado de boa colocação do problema (1) num contexto mais geral

$$
\left|\partial_{t} u\right|^{\rho} \partial_{t t} u-\Delta \partial_{t t} u+\gamma(-\Delta)^{\theta} \partial_{t} u-\alpha \Delta u+\int_{0}^{\infty} \mu(s) \Delta u(t-s) d s+f(u)=h,
$$

$\operatorname{com} \rho \in[0,4)$ e $N=3$.

Agora, observando que problemas de visco-elasticidade com história definem sistemas dinâmicos autônomos (veja Capítulo 4), foi também provado em [2] a existência de um atrator global para o sistema dinâmico associado ao problema (2). Para isso foi preciso adicionar uma dissipação forte do tipo $\Delta u_{t}$. Motivados por esse resultado sobre a existência de atratores, duas versões de atratores não autônomos foram estudados em Qin, Zhang \& Sun [32] e Qin, Feng \& Zhang [33], onde são adicionadas na equação (1) forças do tipo $h=h(x, t)$. No caso autônomo seus resultados melhoram o correspondente em [2] por considerar uma dissipação extra fraca $u_{t}$ ao invés da forte $\Delta u_{t}$.

O nosso trabalho está dividido em quatro capítulos.

- No Capítulo 1 listamos definições e resultados para a compreensão dos capítulos seguintes a serem desenvolvidos.

- No Capítulo 2 apresentamos o conceito de visco-elasticidade com história, seguindo os textos de Pata \& Zucchi [30] e Conti, Marchini \& Pata [11].

- No Capítulo 3 apresentamos mais detalhadamente a prova da boa colocação do problema correspondente à equação (1) seguindo de perto o trabalho de Conti, Marchini \& Pata [11].

- No Capítulo 4 provamos um novo teorema de existência de atratores globais para a equação (1) com $\rho \in(0,4]$. Nosso resultado explora apenas a dissipação dada pelo 
termo da memória. Não necessitamos de dissipações extras tais como $u_{t}$ ou $\Delta u_{t}$. Dessa forma nosso resultado melhora os resultados anteriores em [2, 32, 33].

Sobre a metodologia, observamos que o sistema dinâmico associado ao nosso problema é tipicamente hiperbólica, assim, preferimos seguir a metodologia apresentada por Chueshov \& Lasiecka [9] [10] onde usamos uma desigualdade de estabilização como em [2]. Os resultados do Capítulo 4 serão apresentados em forma de artigo na referência [23]. 


\section{Capítulo 1}

\section{Preliminares}

Neste capítulo apresentamos alguns resultados sobre os espaços de Sobolev, os espaços de Bochner com peso, os sistemas dinâmicos e as soluções suaves o qual nos brindará uma boa base para o desenvolvimento deste trabalho.

\subsection{Espaços de Sobolev}

Apresentaremos a seguir definições e teoremas relacionados aos Espaços de Sobolev. Para maior informação, o leitor interessado pode consultar Adams \& Fournier [1] e Brézis [4].

Definição 1.1: Seja $\Omega \in \mathbb{R}^{n}$ um conjunto aberto. Representa-se por $E^{p}(\Omega), 1 \leq p<$ $+\infty$, o espaço vetorial constituído pelas funções $f: \Omega \rightarrow \mathbb{R}$ mensuráveis, cuja potencia $p$, $|f|^{p}$ é Lebesgue integrável, isto é:

$$
E^{p}(\Omega)=\left\{f: \Omega \rightarrow \mathbb{R} ; f \text { é mensurável e } \int_{\Omega}|f(x)|^{p} d x<\infty\right\}
$$

Define-se em $E^{p}(\Omega)$ a relação $\sim$ dada por:

$$
f \sim g \Leftrightarrow f=g \text { quase sempre em } \Omega .
$$

Notemos que $\sim$ é uma relação de equivalência. Assim, faz sentido considerar o quociente de $E^{p}(\Omega)$ por $\sim$. A coleção das classes de equivalência obtida por $\frac{E^{p}(\Omega)}{\sim}$ forma um espaço vectorial, que denotaremos por:

$$
L^{p}(\Omega):=\frac{E^{p}(\Omega)}{\sim}
$$

no qual definimos a norma

$$
\|u\|_{L^{p}(\Omega)}=\left(\int_{\Omega}|u(x)|^{p} d x\right)^{\frac{1}{p}}, \text { onde } u \in L^{p}(\Omega) .
$$


Definição 1.2: Uma função mensurável $u: \Omega \rightarrow \mathbb{R}$ é dita essencialmente limitada se existe $C>0$ tal que $|u(x)| \leq C$ quase sempre (q.s) em $x \in \Omega$. A coleção das classes de equivalência das funções definidas em $\Omega$ pela relação $\sim$ e essencialmente limitadas é denotada por $L^{\infty}(\Omega)$. Define-se a norma em $L^{\infty}(\Omega)$ por:

$$
\|u\|_{\infty}:=\sup _{x \in \Omega} \operatorname{ess}|u(x)|=\inf \{C>0 ;|u(x)| \leq C \text { q.s. em } x \in \Omega\}
$$

É possível mostrar que $L^{p}(\Omega)$ é um espaço de Banach para $1 \leq p \leq \infty$, além disso, para o caso particular $p=2, L^{2}(\Omega)$ é um espaço de Hilbert cujo produto interno é dado por:

$$
\langle u, v\rangle_{L^{2}(\Omega) \times L^{2}(\Omega)}=\int_{\Omega} u(x) v(x) d x
$$

onde $u$ e $v$ pertencem a $L^{2}(\Omega)$.

Lema 1.3: (Desigualdade de Young). Sejam $1<p, q<\infty \operatorname{com} \frac{1}{p}+\frac{1}{q}=1$. Então,

$$
a b \leq \frac{a^{p}}{p}+\frac{b^{q}}{q}, \quad \forall a, b \geq 0
$$

Lema 1.4: (Desigualdade de Young $\operatorname{com} \epsilon$ ). Sejam $1<p, q<\infty \operatorname{com} \frac{1}{p}+\frac{1}{q}=1$ e $\epsilon>0$ qualquer. Então,

$$
a b \leq \epsilon a^{p}+C(\epsilon) b^{q}, \quad \forall a, b \geq 0,
$$

onde $C(\epsilon)=(\epsilon p)^{-\frac{-q}{p}} q^{-1}$. No caso particular quando $p=q=2$, dita desigualdade reduz-se a

$$
a b \leq \epsilon a^{2}+\frac{1}{4 \epsilon} b^{2}, \quad \forall a, b \geq 0 .
$$

Teorema 1.5: (Desigualdade de Hölder). Sejam $u \in L^{p}(\Omega)$ e $v \in L^{p^{\prime}}(\Omega)$, com $1 \leq p<$ $\infty$, com $p^{\prime}$ o expoente conjugado de $p$, ou seja, $\frac{1}{p}+\frac{1}{p^{\prime}}=1$. Então:

$$
u v \in L^{1}(\Omega) \quad e \quad \int_{\Omega}|u(x) v(x)| d x \leq\|u\|_{L^{p}(\Omega)}\|v\|_{L^{p^{\prime}}(\Omega)}
$$

Teorema 1.6: (Desigualdade de Hölder Generalizada). Sejam $1 \leq p_{1}, p_{2}, \ldots, p_{n} \leq \infty$ tais que $\frac{1}{p_{1}}+\frac{1}{p_{2}}+\ldots+\frac{1}{p_{n}}=\frac{1}{r} \leq 1$. Se $u_{i} \in L^{p_{i}}(\Omega)$ para $i=1, \ldots, n$, então:

$$
\begin{gathered}
u:=\prod_{i=1}^{n} u_{i} \in L^{r}(\Omega) \quad e \\
\|u\|_{L^{r}(\Omega)} \leq \prod_{i=1}^{n}\left\|u_{i}\right\|_{L^{p_{i}(\Omega)}} .
\end{gathered}
$$


Teorema 1.7: (Lema de Imersão). Sejam $\Omega \subset \mathbb{R}^{n}$, um conjunto aberto com medida finita, $p$ e q tais que $1 \leq p<q \leq \infty$. Então:

$$
L^{q}(\Omega) \hookrightarrow L^{p}(\Omega)
$$

Definição 1.8: Sejam $\Omega$ um aberto do $\mathbb{R}^{n}, 1 \leq p \leq \infty$ e $m \in \mathbb{N}$. O Espaço de Sobolev de ordem $m$ modelado sobre $L^{p}(\Omega)$, que denotaremos por $W^{m, p}(\Omega)$, é o espaço vetorial das funções em $L^{p}(\Omega)$ cujas derivadas distribucionais de ordem $\alpha$ pertencem a $L^{p}(\Omega)$, para todo multi-indice $\alpha$ com $|\alpha| \leq m$. É dizer:

$$
W^{m, p}(\Omega)=\left\{u \in L^{p}(\Omega) ; D^{\alpha} u \in L^{p}(\Omega), \forall \alpha \text { multi-indice tal que }|\alpha| \leq m\right\} .
$$

Quando $1 \leq p<\infty$, não é difícil mostrar que $W^{m, p}(\Omega)$ é um espaço normado munido da norma:

$$
\|u\|_{W^{m, p}(\Omega)}=\left(\sum_{|\alpha| \leq m}\left\|D^{\alpha} u\right\|_{L^{p}(\Omega)}^{p}\right)^{\frac{1}{p}}
$$

Analogamente, $W^{m, \infty}(\Omega)$ é um espaço normado munido da norma:

$$
\|u\|_{W^{m, \infty}(\Omega)}=\sum_{|\alpha| \leq m}\left\|D^{\alpha} u\right\|_{L^{\infty}(\Omega)} .
$$

Teorema 1.9: Sejam $\Omega$ um aberto do $\mathbb{R}^{n}$ e $m \in \mathbb{N}$.

- Os espaços $W^{m, p}(\Omega)$ são espaços de Banach para $1 \leq p \leq \infty$.

- Os espaços $W^{m, p}(\Omega)$ são espaços reflexivos para $1<p<\infty$.

- Os espaços $W^{m, p}(\Omega)$ são espaços separáveis para $1 \leq p<\infty$.

- No caso particular, quando $p=2, W^{m, 2}(\Omega)$ é um espaço de Hilbert com o produto interno

$$
\langle u, v\rangle_{W^{m, 2}(\Omega) \times W^{m, 2}(\Omega)}=\sum_{|\alpha| \leq m}\left\langle D^{\alpha} u, D^{\alpha} v\right\rangle_{L^{2}(\Omega)},
$$

dito espaço o denotaremos por $H^{m}(\Omega)$.

Definição 1.10: Sejam $\Omega$ um aberto do $\mathbb{R}^{n}, 1 \leq p<\infty$ e $m \in \mathbb{N}$. Definimos o espaço $W_{0}^{m, p}(\Omega)$ como:

$$
W_{0}^{m, p}(\Omega):=\overline{D(\Omega)}^{W^{m, p}(\Omega)},
$$

isto é, como o fecho do espaço das funções teste com respeito à norma em $W^{m, p}(\Omega)$. No caso $p=2$, denotaremos $H_{0}^{m}(\Omega):=\overline{D(\Omega)}^{H^{m}(\Omega)}$. 
Definição 1.11: Sejam $\Omega$ um aberto do $\mathbb{R}^{n}, 1 \leq p<\infty$ e $m \in \mathbb{N}$. Definimos o espaço dual de $W_{0}^{m, p}(\Omega)$ como:

$$
\left[W_{0}^{m, p}(\Omega)\right]^{\prime}=: W^{-m, p^{\prime}}(\Omega)
$$

onde $p$ e $p^{\prime}$ são expoentes conjugados. No caso $\mathrm{p}=2$, denotaremos $\left[H_{0}^{m}(\Omega)\right]^{\prime}=: H^{-m}(\Omega)$.

Teorema 1.12: (Imersões de Sobolev). Seja $\Omega \in \mathbb{R}^{n}$ um dominio e, para $1 \leq k \leq n$, seja $\Omega_{k}$ a interseção de $\Omega$ com o plano de dimensão $k$ em $\mathbb{R}^{n}$. (Se $k=n$ então $\Omega_{k}=\Omega$.) Se $j \geq 0$ e $m \geq 1$ são números inteiros dados e se $1 \leq p<\infty$.

- PARTE I: Suponha que $\Omega$ cumpre a condição do cone.

- Caso A: Se mp $>n$ ou $m=n$ e $p=1$, então:

$$
W^{j+m, p}(\Omega) \hookrightarrow C_{B}^{j}(\Omega)
$$

Alem disso, se $1 \leq k \leq n$, então:

$$
W^{j+m, p}(\Omega) \hookrightarrow W^{j, q}\left(\Omega_{k}\right) \quad \text { para } p \leq q \leq \infty,
$$

e, em particular,

$$
W^{m, p}(\Omega) \hookrightarrow L^{q}(\Omega) \text { para } p \leq q \leq \infty
$$

- Caso B: Se mp =n e $1 \leq k \leq n$, então:

$$
W^{j+m, p}(\Omega) \hookrightarrow W^{j, q}\left(\Omega_{k}\right) \quad \text { para } p \leq q<\infty,
$$

e, em particular,

$$
W^{m, p}(\Omega) \hookrightarrow L^{q}(\Omega) \text { para } p \leq q<\infty .
$$

- Caso C: Se $m p<n$ e, o bem, $n-m p<k \leq n$ ou $p=1$ e $n-m \leq k \leq n$, então:

$$
W^{j+m, p}(\Omega) \hookrightarrow W^{j, q}\left(\Omega_{k}\right) \text { para } p \leq q \leq p *=\frac{k p}{n-m p},
$$

e, em particular,

$$
W^{m, p}(\Omega) \hookrightarrow L^{q}(\Omega) \quad \text { para } p \leq q \leq p *=\frac{k p}{n-m p} .
$$

- PARTE II: Suponha que $\Omega$ satisfaz a condição forte local de Lipschitz. Então o espaço $C_{B}^{j}(\Omega)$ do caso $A$, pode ser substituído pelo espaço menor $C^{j}(\bar{\Omega})$, e as imersões podem ser reescritas como: 
- Se $m p>n>(m-1) p$, então

$$
W^{j+m, p}(\Omega) \hookrightarrow C^{j, \lambda}(\bar{\Omega}) \text { para } 0<\lambda \leq m-\frac{n}{p} .
$$

$-S e n=(m-1) p$, então

$$
W^{j+m, p}(\Omega) \hookrightarrow C^{j, \lambda}(\bar{\Omega}) \quad \text { para } 0<\lambda<1 .
$$

- Se $n=m-1$ e $p=1$, então

$$
W^{j+m, p}(\Omega) \hookrightarrow C^{j, 1}(\bar{\Omega}) .
$$

Lembremos que $C^{j, \lambda}(\bar{\Omega})$ representa o espaço das funções em $C^{j}(\bar{\Omega})$ cujas derivadas de ordem $\lambda$ são $j$-Holder contínuas.

- PARTE 3: Todas as imersões da parte $A$ e $B$ são validas para $\Omega$ um dominio arbitrário se os espaços de Sobolev envolvidos em ditas partes, são substituídas por seus correspondentes $W_{0}$-espaços.

Observação 1.13: No caso da imersão $H_{0}^{1}(\Omega) \hookrightarrow L^{2}(\Omega)$, a melhor constante de dita imersão é dada por $\lambda_{1}$, sendo $\lambda_{1}$ o primeiro autovalor do operado $-\Delta$. Isto é, se $u \in H_{0}^{1}(\Omega)$, então

$$
\lambda_{1}\|u\|_{L^{2}(\Omega)}^{2} \leq\|u\|_{H_{0}^{1}(\Omega)}^{2}
$$

com

$$
\lambda_{1}=\inf _{u \in H_{0}^{1}(\Omega)} \frac{\|\nabla u\|_{L^{2}(\Omega)}^{2}}{\|u\|_{L^{2}(\Omega)}^{2}} .
$$

Teorema 1.14: (Teorema de Rellich-Kondrachov). Sejam $\Omega$ um dominio em $\mathbb{R}^{n}, \Omega_{0}$ um subdominio limitado de $\Omega$, e $\Omega_{0}^{k}$ a interseção de $\Omega_{0}$ com um plano $k$-dimensional em $\mathbb{R}^{n}$. Sejam $j \geq 0$ e $m \geq 1$ inteiros, e $1 \leq p<\infty$.

- PARTE I: Se $\Omega$ satisfaz a condição do cone e $m p \leq n$, então seguem as seguintes imersões compactas:

$$
\begin{gathered}
W^{j+m, p}(\Omega) \stackrel{c}{\hookrightarrow} W^{j, q}\left(\Omega_{0}^{k}\right) \quad \text { se } 0<n-m p<k \leq n \text { e } 1 \leq q<\frac{k p}{n-m p} . \\
W^{j+m, p}(\Omega) \stackrel{c}{\hookrightarrow} W^{j, q}\left(\Omega_{0}^{k}\right) \quad \text { se } n=m p, 1 \leq k \leq n \text { e } 1 \leq q<\infty .
\end{gathered}
$$

- PARTE II: Se $\Omega$ satisfaz a condição do cone e $m p>n$, então seguem as seguintes imersões compactas:

$$
\begin{gathered}
W^{j+m, p}(\Omega) \stackrel{c}{\hookrightarrow} C_{B}^{j}\left(\Omega_{0}\right) \\
W^{j+m, p}(\Omega) \stackrel{c}{\hookrightarrow} W^{j, q}\left(\Omega_{0}^{k}\right) \quad \text { se } 1 \leq q<\infty .
\end{gathered}
$$


- PARTE III: Se $\Omega$ satisfaz a condição forte local de Lipschitz, então seguem as seguintes imersões compactas:

$$
\begin{gathered}
W^{j+m, p}(\Omega) \stackrel{c}{\hookrightarrow} C^{j}\left(\overline{\Omega_{0}^{k}}\right) \quad \text { se } 0 m p>n . \\
W^{j+m, p}(\Omega) \stackrel{c}{\hookrightarrow} C^{j, \lambda}\left(\overline{\Omega_{0}^{k}}\right) \text { se } m p>n \geq(m-1) p \text { e } 0<\lambda<m-\frac{n}{p} .
\end{gathered}
$$

- PARTE IV: Se $\Omega$ é um dominio arbitrário em $\mathbb{R}^{n}$, então seguem as seguintes imersões compactas:

$$
\begin{gathered}
W_{0}^{j+m, p}(\Omega) \stackrel{c}{\hookrightarrow} W^{j, q}\left(\Omega_{0}^{k}\right) \quad \text { se } 0<n-m p<k \leq n \text { e } 1 \leq q<\frac{k p}{n-m p} . \\
W_{0}^{j+m, p}(\Omega) \stackrel{c}{\hookrightarrow} C^{j, \lambda}\left(\overline{\Omega_{0}^{k}}\right) \text { se } m p>n \geq(m-1) p \text { e } 0<\lambda<m-\frac{n}{p} .
\end{gathered}
$$

Teorema 1.15: (Desigualdade de Poincaré). Seja $\Omega \subset \mathbb{R}^{n}$ um domínio limitado e $1 \leq p<\infty$. Então existe uma constante $C=C(p,|\Omega|)>0$ tal que:

$$
\|u\|_{L^{p}(\Omega)} \leq C\|\nabla u\|_{L^{p}(\Omega)}, \quad \forall u \in W_{0}^{m, p}(\Omega)
$$

Definição 1.16: Seja $X$ um espaço de Banach e $1 \leq p<\infty$. Denotaremos por:

$$
\left(L^{p}(0, \tau ; X) ;\|\cdot\|_{L^{p}(0, \tau ; X)}\right)
$$

ao espaço de Banach das funções vetoriais mensuráveis $u:(0, \tau) \rightarrow X$, tais que $\|u(t)\|_{X}$ pertence a $L^{p}(0, \tau)$, munido da norma

$$
\|u\|_{L^{p}(0, \tau ; X)}=\left(\int_{0}^{\tau}\|u(t)\|_{X}^{p} d t\right)^{\frac{1}{p}}
$$

No caso $p=\infty$, denotaremos por $\left(L^{\infty}(0, \tau ; X) ;\|\cdot\|_{L^{\infty}(0, \tau ; X)}\right)$ ao espaço de Banach das funções vetoriais mensuráveis $u:(0, \tau) \rightarrow X$, tais que $\|u(t)\|_{X}$ pertence a $L^{\infty}(0, \tau)$, munido da norma

$$
\|u\|_{L^{\infty}(0, \tau ; X)}=\sup _{t \in(0, \tau)} e s s\|u(t)\|_{X}
$$

Uma ferramente que vai ser fortemente usada no trabalho sera o Teorema de Compacidade de Simon [34], que é uma extensão do Teorema de Aubin-Lions.

Teorema 1.17: (Teorema de Compacidade de Simon). Sejam X, B, Y espaços de Banach tais que $X \stackrel{c}{\hookrightarrow} B \hookrightarrow Y$. Suponhamos que:

- $\left(u_{n}\right)$ é limitada em $L^{\infty}(0, \tau ; X)$ 
- $\left(\partial_{t} u_{n}\right)$ é limitada em $L^{r}(0, \tau ; Y), r>1$.

Então, $\left(u_{n}\right)$ é relativamente compacto em $C(0, \tau ; B)$.

Lema 1.18: (Desigualdade de Gronwall). Sejam $\alpha \geq 0$ uma constante, $\beta \in L^{1}(a, b)$ e $\phi \in L^{\infty}(a, b)$ tais que $\beta>0$ e $\phi \geq 0$. Se

$$
\phi(t) \leq \alpha+\int_{a}^{b} \beta(s) \phi(s) d s, \quad a \leq t \leq b
$$

então

$$
\phi(t) \leq \alpha e^{\int_{a}^{b} \beta(s) d s}, \quad a \leq t \leq b .
$$

\subsection{Espaços de Bochner com peso}

Nesta seção faremos uma revisão dos espaços de Bochner, que sera fundamental para poder definir o "espaço de história"que esta envolto em nossa equação. Para maior detalhe se remite ao lector consultar os trabalhos de Adams \& Fournier [1], Yosida [39], Butzer [5] e Folland [14].

Seja $X$ um espaço de Banach com norma $\|$. $\|_{X}$ e seja $f$ uma função definida no intervalo $(a, b)$ em $\mathbb{R}$ (que pode ser finito) e tendo valores em $X$. Alem disso, seja $\nu$ uma medida em $(a, b)$ dada por $d \nu(s)=\mu(s) d s$ onde $\mu$ é continua e positiva em $(a, b)$.

Definição 1.19: (Integral de Bochner). Se $A_{1}, A_{2}, \ldots, A_{k}$ é uma coleção finita de subconjuntos disjuntos de $(a, b)$ tendo cada um medida $\nu$ finita, e se $x_{1}, x_{2}, \ldots, x_{k}$ é um conjunto de elementos de $X$, chamaremos à função $f$ dada por

$$
f(t)=\sum_{i=1}^{k} \chi_{A_{i}} x_{i}, \quad a<t<b,
$$

como "função simples" sobre $(a, b)$ en $X$. Para este tipo de funções simples definimos a integral de $f$ como

$$
\int_{a}^{b} f(s) d \nu(s)=\sum_{i=1}^{k} \nu\left(A_{i}\right) x_{i}=\sum_{i=1}^{k}\left(\int_{A_{i}} \mu(s) d s\right) x_{i}
$$

a qual independe da escolha de dita partição.

Agora, seja $f$ uma função arbitraria definida sobre $(a, b)$ em $X$. Diremos que " $f$ é (fortemente) medível" em $(a, b)$, se existe uma sequencia $f_{i}$ de funções simples com suporte em $(a, b)$ tal que

$$
\lim _{j \rightarrow \infty}\left\|f_{j}(t)-f(t)\right\|_{X} \quad \text { q.s. em }(a, b)
$$


Então, definimos a "Integral de Bochner de $f$ em $(a, b)$ " como

$$
\int_{a}^{b} f(s) d \nu(s)=\lim _{j \rightarrow \infty} \int_{a}^{b} f_{j}(s) d \nu(s)
$$

Analogamente, como a integral das funções simples, a integral anterior independe da escolha da sequencia de funções simples que aproximam $f$.

Teorema 1.20: Uma função $f$ é Bochner integrável em $(a, b) \Leftrightarrow$ a função $\| f($. $) \|_{X}$ é integrável em $(a, b)$.

Definição 1.21: (Espaço $\left.L_{\mu}^{p}(a, b ; X)\right)$.

- Se $1 \leq p<\infty$, diremos que $f \in\left(L_{\mu}^{p}(a, b ; X) ; \|\right.$. $\left.\|_{L_{\mu}^{p}(a, b ; X)}\right)$ se

$$
\|f\|_{L_{\mu}^{p}(a, b ; X)}=\left(\int_{a}^{b}\|f(s)\|_{X}^{p} d \nu(s)\right)^{\frac{1}{p}}=\left(\int_{a}^{b} \mu(s)\|f(s)\|_{X}^{p} d s\right)^{\frac{1}{p}}<\infty .
$$

- Se $p=\infty$, diremos que $f \in\left(L_{\mu}^{\infty}(a, b ; X) ;\|\cdot\|_{L_{\mu}^{\infty}(a, b ; X)}\right)$ se

$$
\|f\|_{L_{\mu}^{\infty}(a, b ; X)}=\sup _{a<s<b} e s s\|f(s)\|_{X}<\infty .
$$

\section{Teorema 1.22:}

- $O$ espaço $L_{\mu}^{p}(a, b ; X)$ para $1 \leq p \leq \infty$ é de Banach.

- Se o espaço $X$ é de Hilbert então o espaço $L_{\mu}^{2}((a, b) ; X)$ é de Hilbert, e o produto interno é dado por

$$
\langle u, v\rangle_{L_{\mu}^{2}((a, b) ; X) \times L_{\mu}^{2}((a, b) ; X)}=\int_{a}^{b} \mu(s)\langle u, v\rangle_{X \times X} d s
$$

\subsection{Sistemas dinâmicos e atratores globais}

Nesta seção faremos uma revisão sobre alguns tópicos referentes à teoria dos sistemas dinâmicos definidos por um semigrupo fortemente continuo de um espaço de Banach $X$. Repassaremos os resultados apresentados em Teman [36], Hale [16], Babin \& Vishik [3], e faremos um estudo mais perto aos trabalhos de Chueshov \& Lasiecka [9], [10].

Definição 1.23: Uma família de operadores não necessariamente lineares $S(t)_{t \geq 0}$, fortemente contínua em $X$, é chamado de $C_{0}-$ semigrupo se:

- $S(0)=I$ (Operador identidade de $X)$. 
- $S(t+s)=S(t) S(s)$ para cada $t, s \geq 0$.

- A aplicação $[0, \infty) \times X \ni(t, x) \longmapsto S(t)(x) \in X$ é continua para cada $x \in X$ dado. O par $(X, S(t))$ também é chamado sistema dinâmico, definido pelo semigrupo $S(t)$.

Definição 1.24: Sejam $(X, S(t))$ um sistema dinâmico e $\mathcal{A}$ um subconjunto de $X$.

- Diremos que $\mathcal{A}$ é positivamente invariante pelo ação semigrupo $S(t)$, quando $S(t) \mathcal{A} \subset$ $\mathcal{A}$ para todo $t \geq 0$.

- Diremos que $\mathcal{A}$ é invariante pelo ação do semigrupo $S(t)$, quando $S(t) \mathcal{A}=\mathcal{A}$ para todo $t \geq 0$.

Definição 1.25: (Definição de Atrator Global). Seja $(X, S(t))$ um sistema dinâmico. Diremos que um subconjunto $\mathcal{A} \subset X$ é um Atrator Global de $(X, S(t))$ quando:

- $\mathcal{A}$ é um conjunto fechado e limitado,

- $\mathcal{A}$ é un conjunto invariante por $\mathrm{S}(\mathrm{t})$,

- $\mathcal{A}$ atrai qualquer subconjunto limitado de $X$ pela ação do semigrupo $S(t)$, isto é, para todo limitado $B \subset X$,

$$
\lim _{t \rightarrow \infty} \operatorname{dist}_{H}(S(t) B, \mathcal{A})=0
$$

onde $\operatorname{dist}_{H}(A, B)$ é a semi-distancia de Hausdorff entre os subconjuntos $A, B \subset X$ e é dada por

$$
\operatorname{dist}_{H}(A, B):=\sup _{x \in A} d_{X}(x, B)=\sup _{x \in A} \inf _{y \in B} d(x, y) .
$$

Definição 1.26: (Conjunto Absorvente). Seja $(X, S(t))$ um sistema dinâmico. Um conjunto $\mathcal{B} \subset X$ é chamado de "Conjunto Absorvente de $(X, S(t))$ " se, para qualquer subconjunto limitado $B \subset X$, existe $\tau_{0}=\tau_{0}(B) \geq 0$ tal que

$$
S(t) B \subset \mathcal{B}, \quad \forall t \geq \tau_{0} .
$$

Quando um sistema dinâmico $(X, S(t))$ possui um conjunto absorvente limitado, dizemos que $(X, S(t))$ é um sistema dinâmico dissipativo.

Definição 1.27: (Compacidade Assintótica). Um sistema dinâmico $(X, S(t))$ é dito "Assintoticamente Compacto" se existe um conjunto $K \subset X$ compacto tal que para cada conjunto limitado $B \subset X$,

$$
\lim _{t \rightarrow \infty} \operatorname{dist}_{H}(S(t) B, K)=0
$$


Agora apresentaremos um resultado conhecido sobre a existência de atratores globais para semigrupos que sera a pedra angular para a prova da existência do atrator global em nosso trabalho, o leitor interessado pode consultar Miranville \& Zelik [28].

Teorema 1.28: Seja $(X, S(t))$ um sistema dinâmico. Então, diremos que dito sistema dinâmico possui atrator global $\mathcal{A}$ se, e somente se, é assintoticamente compacto e dissipativo. Além disso, em caso afirmativo, se $\mathcal{B}$ denota a coleção de todos os subconjuntos limitados não vazios de $X$, então o atrator $\mathcal{A}$ vem dado por

$$
\mathcal{A}=\bigcup_{B \in \mathcal{B}} w(B)
$$

onde $w(B)$ é o conjunto w-limite de $B$ e é dado por

$$
\begin{gathered}
w(B)=\left\{x \in X: \text { existem sequencias }\left(t_{n}\right)_{n \in \mathbb{N}} \text { em } \mathbb{R}^{+} \text {com } t_{n} \rightarrow \infty\right. \\
\left.e\left(x_{n}\right)_{n \in \mathbb{N}} \text { em } B \text {, tais que } x=\lim _{n \rightarrow \infty} S\left(t_{n}\right) x_{n}\right\} .
\end{gathered}
$$

Nosso sistema envolto no presente trabalho, na verificação da compacidade assintótica pode ser bastante difícil. Por isso, introduzimos o conceito de regularidade assintótica, que sera usado junto aos resultados feitos por Chueshov \& Lasiecka [10], onde apontam que num sistema dissipativo os conceitos de compacidade assintótica e regularidade assintótica são equivalente.

Definição 1.29: (Regularidade Assintótica). Um sistema dinâmico $(X, S(t))$ é "Assintoticamente Regular" se, para qualquer conjunto limitado e positivamente invariante $B \subset X$, existe um conjunto compacto $K \subset \bar{B}^{X}$ tal que

$$
\lim _{t \rightarrow \infty} \operatorname{dist}_{H}(S(t) B, K)=0 .
$$

Teorema 1.30: (Chueshov \& Lasiecka [10] 7.1.4 Proposition). Assumamos que X é um espaço de Banach e $(X, S(t))$ um sistema dinâmico dissipativo. Então as seguintes afirmações são equivalentes:

- $(X, S(t))$ é assintoticamente compacto.

- $(X, S(t))$ é assintoticamente regular.

Teorema 1.31: (Chueshov \& Lasiecka [10] 7.1.11 Proposition) Seja $(X, S(t))$ um sistema dinâmico. Suponhamos que para algum conjunto limitado positivamente invariante $B \subset X$ e para algum $\epsilon>0$, exista $T=T(\epsilon, B)$ tal que

$$
\left\|S(T) z^{1}-S(T) z^{2}\right\|_{X} \leq \epsilon+\phi_{T}\left(z^{1}, z^{2}\right), \quad \forall z^{1}, z^{2} \in B
$$


onde $\phi_{T}: B \times B \rightarrow \mathbb{R}$ satisfaz

$$
\liminf _{n \rightarrow \infty} \liminf _{m \rightarrow \infty} \phi_{T}\left(z^{n}, z^{m}\right)=0
$$

para alguma sequencia $\left(z^{n}\right)$ em $B$. Então, $(X, S(t))$ é um sistema dinâmico assintoticamente regular.

\subsection{Soluções fracas (mild solutions)}

Nesta seção faremos uma revisão sobre as soluções suaves no sentido de A. Pazy [31] Consideremos o problema de valor inicial não-homogêneo

$$
\begin{gathered}
\frac{d u(t)}{d t}=A u(t)+f(t), \quad t>0 \\
u(0)=x
\end{gathered}
$$

$\operatorname{com} f:[0, \tau) \rightarrow X$, onde $A$ é um gerador infinitesimal de um $C_{0}$-semigrupo $\Sigma(t)$, tal que a correspondente equação homogênea tem uma solução única para cada valor $x \in \operatorname{dom}(A)$.

Definição 1.32: A função $u:[0, \tau] \rightarrow X$ é uma "solução clássica" de $(1.2)$ em $[0, \tau)$, se $u$ é continua em $[0, \tau)$, continuamente diferenciável em $(0, \tau), u(t) \in \operatorname{dom}(A)$ para todo $t \in(0, \tau)$ e $(1.2)$ é satisfeito em $[0, \tau)$.

Teorema 1.33: Se $f \in L^{1}(0, \tau ; X)$, então para cada $x \in X$ o problema de valor inicial (1.2), tem como máximo uma solução (clássica) que é dada por

$$
u(t)=\Sigma(t) x+\int_{0}^{t} \Sigma(t-s) f(s) d s .
$$

Definição 1.34: Seja $A$ um gerador infinitesimal de um $C_{0}$-semigrupo $\Sigma(t)$. Se $x \in X$ e $f \in L^{1}(0, \tau ; X)$, então a função $u \in C([0, \tau] ; X)$ é dada por

$$
u(t)=\Sigma(t) x+\int_{0}^{t} \Sigma(t-s) f(s) d s, \quad t \in[0, \tau] .
$$

Dita função $u$ é chamada fraca (mild solution) de (1.2) em [0, $\tau]$.

\section{Observação 1.35:}

- Não toda solução suave é solução clássica.

- É claro que se $f \in L^{1}(0, \tau ; X)$, o problema (1.2) tem uma única solução fraca. 


\section{Capítulo 2 \\ Equação viscoelástico não-linear com história}

Neste capítulo, estudamos principalmente as bases para a boa colocação do problema correspondente à equação (1). Explicaremos detalhadamente o entorno funcional que envolve o problema e como vamos a enfocar a "história" envolvida nessa equação, dado que seguiremos o argumento de Dafermos [12]. Além disso, faremos um estudo detalhado do trabalho de Conti, Marchini \& Pata [11], tanto neste capítulo como no Capítulo 3.

Enunciemos as hipóteses que se serão usadas ao longo do trabalho. Lembramos que a nossa equação

$$
\left|\partial_{t} u\right|^{\rho} \partial_{t t} u-\Delta \partial_{t t} u-\alpha \Delta u+\int_{0}^{\infty} \mu(s) \Delta u(t-s) d s+f(u)=h
$$

está definida numa região limitada $\Omega \subset \mathbb{R}^{3}$ com fronteira $\partial \Omega$ suave e com condições de Dirichlet, isto é $\left.u(x, t)\right|_{x \in \partial \Omega}=0$. Ademais $\rho \in[0,4)$ e $\alpha>0$ um parâmetro fixo.

Assumiremos as seguintes hipóteses:

(H1) A equação possui condições iniciais

$$
u(x, 0)=u_{0}, \quad \partial_{t} u(x, 0)=v_{0},\left.\quad u(x,-s)\right|_{s \in \mathbb{R}^{+}}=\phi_{0}(s) \quad \forall x \in \Omega,
$$

onde $u_{0}, v_{0}: \Omega \rightarrow \mathbb{R}$ e $\phi_{0}: \Omega \times \mathbb{R}^{+} \rightarrow \mathbb{R}$ funções dadas. Notemos que a função $\phi_{0}$ nos da informação sobre a história passada de $u$.

(H2) A função $h \in L^{2}(\Omega)$ é independente do fator temporal e representa alguma força externa ao sistema.

(H3) A função $f$ é possivelmente não linear, localmente Lipschitz e satisfaz

$$
f(0)=0
$$


que cumpre a condição de crescimento

$$
|f(u)-f(v)| \leq c|u-v|\left(1+|u|^{q}+|v|^{q}\right),
$$

onde, $c$ é uma constante e $q \in[0,4)$,

e que cumpre a condição de dissipação

$$
\liminf _{|u| \rightarrow \infty} \frac{f(u)}{u}>-\lambda_{1}
$$

onde $\lambda_{1}>0$ é o primeiro autovalor de $-\Delta$. Além disso, seja $\widehat{f}(u)=\int_{0}^{u} f(y) d y$, então também pedimos

$$
f(u) u-\widehat{f}(u) \geq-\frac{\beta}{2} u^{2}-m_{f} \quad \forall u \in \mathbb{R},
$$

onde $m_{f}$ é uma constante que só depende de $f$ e $\beta \in\left(0, \lambda_{1}\right)$.

(H4) O núcleo da convolução (o memória) $\mu$ é uma função que satisfaz

$$
\mu \in C^{1}\left(\mathbb{R}^{+}\right) \cap L^{1}\left(\mathbb{R}^{+}\right), \quad 0<\mu(s)<\infty, \quad \mu^{\prime}(s) \leq 0,
$$

e que existem $k_{0}, k_{1}>0, k_{2}>0$ tais que

$$
\begin{gathered}
\int_{0}^{\infty} \mu(s) d s=k_{0} \in(0, \alpha), \\
\mu^{\prime}(s) \leq-k_{1} \mu(s), \quad \forall s \in \mathbb{R}^{+} .
\end{gathered}
$$

$\mathrm{e}$

$$
\int_{0}^{\infty}-\mu^{\prime}(s) d s=k_{2}, \quad 0<k_{2}<\infty
$$

Observação 2.1: Notemos que das hipóteses anteriores temos que $k_{2} \geq k_{0} k_{1}$.

Observação 2.2: Para facilitar as contas, sim perda de generalidade, assumiremos que

$$
\alpha-k_{0}=1
$$

\subsection{Entorno funcional}

Agora construiremos um espaço "bom" onde serão definidas as soluções fracas da equação (1). Dado que o problema envolve uma convolução referente à história do material, vamos ter que definir uma nova variável que habitara dentro de um espaço de Bochner com peso que se apresentara nesta mesma seção. 
Observação 2.3: Para facilitar as contas ao largo do trabalho, usaremos certas notações que explicaremos acontinuação:

- Dado que nosso problema envolve ao operador Laplaciano, denotaremos por

$$
A:=-\Delta
$$

notemos ademais que

$$
\operatorname{dom}(A)=H^{2}(\Omega) \cap H_{0}^{1}(\Omega) \stackrel{c}{\hookrightarrow} L^{2}(\Omega) .
$$

- Denotaremos a norma no espaço $L^{p}(\Omega)$, para todo $p \in[1, \infty]$ como:

$$
\|u\|_{p}:=\|u\|_{L^{p}(\Omega)}, \quad \forall u \in L^{p}(\Omega) .
$$

alem disso, denotamos o produto interno em $L^{2}(\Omega)$ como

$$
(u, v):=\langle u, v\rangle_{L^{2}(\Omega) \times L^{2}(\Omega)}, \quad \forall u, v \in L^{2}(\Omega)
$$

- Dadas as observações anteriores notemos que podemos denotar a norma no espaço $H_{0}^{1}(\Omega)$ como

$$
\|\nabla u\|_{2}:=\|u\|_{H_{0}^{1}(\Omega)}, \quad \forall u \in H_{0}^{1}(\Omega),
$$

alem disso, denotamos o produto interno neste espaço por

$$
\langle u, v\rangle_{1}:=\langle u, v\rangle_{H_{0}^{1}(\Omega) \times H_{0}^{1}(\Omega)}=(\nabla u, \nabla v)=(A u, v), \quad \forall u, v \in H_{0}^{1}(\Omega) .
$$

- Adualidade entre $H^{-1}(\Omega)$ e $H_{0}^{1}(\Omega)$ será denotada por

$$
\langle f, u\rangle:=\langle f, u\rangle_{H^{-1}(\Omega) \times H_{0}^{1}(\Omega)}, \quad \forall f \in H^{-1}(\Omega), \quad u \in H_{0}^{1}(\Omega) .
$$

Definição 2.4: Dada a hipóteses (2.5), definimos a medida $\nu$ em $\left(\mathbb{R}^{+}, \mathcal{B}_{\mathbb{R}^{+}}\right)$dada por

$$
d \nu(s)=\mu(s) d s
$$


Definição 2.5: (Espaço de história). Dado $\nu$ como na definição anterior, definimos o espaço de Bochner com peso

$$
\mathcal{M}:=L_{\mu}^{2}\left(\mathbb{R}^{+} ; H_{0}^{1}(\Omega)\right)
$$

chamado "espaço de história". Alem disso, dado que $H_{0}^{1}(\Omega)$ é um espaço de Hilbert, o espaço $\mathcal{M}$ munida do seguinte produto interno e norma

$$
\langle\eta, \xi\rangle_{\mathcal{M}}:=\int_{0}^{\infty} \mu(s)\langle\eta(s), \xi(s)\rangle_{1} d s, \quad\|\eta\|_{\mathcal{M}}^{2}:=\int_{0}^{\infty} \mu(s)\|\nabla \eta\|_{2}^{2} d s, \quad \forall \eta, \xi \in \mathcal{M}
$$

é um espaço de Hilbert.

Agora, como já se falou anteriormente, nós seguiremos o enfoque de Dafermos, e para isso é preciso definir o seguinte semigrupo traslação a direita.

Definição 2.6: Definimos o seguinte semigrupo $\Sigma(t)$ em $\mathcal{M}$ dado por

$$
[\Sigma(t) \eta](s)= \begin{cases}0 & \text { se } \quad 0<s \leq t \\ \eta(s-t) & \text { se } \quad s>t\end{cases}
$$

Dito semigrupo sera chamado "Semigrupo Traslação a Direita".

É claro observar que dito semigrupo cumpre com as seguintes propriedades:

- O semigrupo $\Sigma(t)$ é um semigrupo $C_{0}$-semigrupo de operadores lineares.

- O gerador infinitesimal do semigrupo $\Sigma(t)$ é dado pelo operador lineal $T: \operatorname{dom}(T) \rightarrow$ $\mathcal{M}$ onde

$$
T \eta=-\eta^{\prime}, \quad \operatorname{dom}(T)=\left\{\eta \in \mathcal{M}: \eta^{\prime} \in \mathcal{M}, \eta(0)=0\right\}
$$

Teorema 2.7: Dada o semigrupo anterior $\Sigma(t)$, com o gerador infinitesimal $T$, se cumpre que

$$
\langle T \eta, \eta\rangle_{\mathcal{M}} \leq 0, \quad \forall \eta \in \operatorname{dom}(T)
$$


Demonstração. Seja $\eta \in \operatorname{dom}(T)$, então temos que

$$
\begin{aligned}
\langle T \eta, \eta\rangle_{\mathcal{M}}=\left\langle-\eta^{\prime}, \eta\right\rangle_{\mathcal{M}} & =\int_{0}^{\infty} \mu(s)\left\langle-\eta^{\prime}, \eta\right\rangle_{1} d s \\
& =-\int_{0}^{\infty} \mu(s)\left[\int_{\Omega} \sum_{i=1}^{3} \frac{\partial \eta^{\prime}}{\partial x_{i}} \frac{\partial \eta}{\partial x_{i}} d x\right] d s \\
& =-\int_{\Omega}\left[\int_{0}^{\infty} \mu(s) \sum_{i=1}^{3} \frac{\partial \eta^{\prime}}{\partial x_{i}} \frac{\partial \eta}{\partial x_{i}} d s\right] d x \\
& =-\frac{1}{2} \int_{\Omega}\left[\int_{0}^{\infty} \mu(s) \frac{d}{d s}\left(\sum_{i=1}^{3}\left|\frac{\partial \eta}{\partial x_{i}}\right|^{2}\right) d s\right] d x \\
& =-\frac{1}{2} \int_{\Omega}\left[\int_{0}^{\infty} \mu(s) \frac{d}{d s}\|\nabla \eta(s)\|_{\mathbb{R}^{3}}^{2} d s\right] d x \\
& =\frac{1}{2} \int_{\Omega}\left[\int_{0}^{\infty} \mu^{\prime}(s)\|\nabla \eta(s)\|_{\mathbb{R}^{3}}^{2} d s\right] d x-\int_{\Omega}\left[\mu(s)\|\nabla \eta(s)\|_{\mathbb{R}^{3}}^{2}\right]_{0}^{\infty} d x \\
& =\frac{1}{2} \int_{\Omega}\left[\int_{0}^{\infty} \mu^{\prime}(s)\|\nabla \eta(s)\|_{\mathbb{R}^{3}}^{2} d s\right] d x,
\end{aligned}
$$

agora por (2.5) temos que $\mu^{\prime} \leq 0$, então

$$
\langle T \eta, \eta\rangle_{\mathcal{M}} \leq 0
$$

\subsection{Problema com história}

Seguindo com o enfoque no marco da história para nossa equação, vamos a "modificar" nossa equação por meio de um "deslocamento" que gerará uma nova variável. Seguiremos, como se falo antes, o trabalho de Dafermos [12]. Também podemos encontrar o mesmo tipo de enfoque em Giorgi, Grasseli \& Pata [15], Pata \& Zucchi [30] e Conti, Marchini \& Pata [11].

Definição 2.8: Seja $\Omega$ um domínio limitado de $\mathbb{R}^{3}$, com fronteira $\partial \Omega$ suave. Seja $\eta$ o "Deslocamento Relativo da História do Sistema" , o qual é dado por

$$
\eta=\eta^{t}(x, s)=u(x, t)-u(x, t-s), \quad(x, s) \in \Omega \times \mathbb{R}^{+}, t \geq 0 .
$$

A pergunta é, por que a necessidade de definir dito deslocamento?. A necessidade é escrever de uma "melhor" maneira o termo da convolução em nossa equação. Pois notemos que se derivamos dito deslocamento formalmente com relação a $t$ e $s$ obtemos

$$
\eta_{t}^{t}(x, s)=-\eta_{s}^{t}(x, s)+u_{t}(x, t), \quad(x, s) \in \Omega \times \mathbb{R}^{+}, t \geq 0,
$$


assim o termo original da história (convolução), é reescrita como

$$
\int_{0}^{\infty} \mu(s) \Delta u(t-s) d s=\left(\int_{0}^{\infty} \mu(s) d s\right) \Delta u-\int_{0}^{\infty} \mu(s) \Delta \eta^{t}(s) d s .
$$

Agora reescrevendo a equação (1) com este cambio, temos que

$$
\left|\partial_{t} u\right|^{\rho} \partial_{t t} u-\Delta \partial_{t t} u-\alpha \Delta u+\int_{0}^{\infty} \mu(s) \Delta u(t-s) d s+f(u)=h,
$$

torna-se

$$
\left|\partial_{t} u\right|^{\rho} \partial_{t t} u-\Delta \partial_{t t} u-\left(\alpha-\int_{0}^{\infty} \mu(s) d s\right) \Delta u-\int_{0}^{\infty} \mu(s) \Delta \eta^{t}(s) d s+f(u)=h
$$

alem disso, pela observação (2.9) e tendo em quenta que $A=-\Delta$, temos que finalmente a equação (1) se escreve como

$$
\left|u_{t}\right|^{\rho} u_{t t}+A u_{t t}+A u+\int_{0}^{\infty} \mu(s) A \eta^{t}(s) d s+f(u)=h
$$

Observação 2.9: Notemos que trocamos de notação para a derivada de $u$ respeito de $t$, isto é só para não sobrecarregar as notações ao largo do trabalho. Alem disso, omitimos também o par de variáveis $(x, t) \in[0, \infty)$.

Assim, para terminar de reescrever nossa equação em termos de este deslocamento, vamos a definir a seguinte condição inicial para $\eta$.

Definição 2.10: Seja $\phi_{0}: \Omega \times \mathbb{R}^{+} \rightarrow \mathbb{R}$ como na hipóteses (H1). Definimos a condição inicial $(t=0)$ para nosso deslocamento relativo da história do sistema como

$$
\eta^{0}(x, s):=u_{0}(x, 0)-u(x,-s)=u_{0}(x, 0)-\phi_{0}(x, s), \quad(x, s) \in \Omega \times \mathbb{R}^{+} .
$$

Observação 2.11: Notemos que definindo assim a condição inicial, temos certa compatibilidade com a equação (2.15), isto nos permitirá trabalhar com um sistema de equações, referentes a $u$ e $\eta$ em lugar de só a equação (1).

Agora a pergunta é, em que espaço se encontra dito deslocamento relativo?. É aqui onde entra nosso espaço da história $\mathcal{M}$, para olhar melhor isto estudemos mais de cerca a função $\eta$.

Notemos que em realidade nossa função $\eta$ "trabalha" da seguinte maneira

$$
\eta:[0, \infty) \times \Omega \times \mathbb{R}^{+} \rightarrow \mathbb{R}
$$


tal que

$$
\eta^{t}(x, s):=\eta(t, x, s)=u(x, t)-u(x, t-s) .
$$

Notemos que se fixamos $t \in[0, \infty)$ claramente temos

$$
\eta^{t}: s \mapsto \eta^{t}(\cdot, s) \in H_{0}^{1}(\Omega),
$$

Ademais, como olhamos na equação (2.16) vamos a ter termos do tipo

$$
\int_{0}^{\infty} \mu(s) A \eta^{t}(x, s) d s
$$

assim que precisamos de um espaço "bom", onde se tenha certo controle em expressões como a anterior. Para isso, fixando $t \in[0, \infty)$, temos que $\eta^{t}$ pertence ao espaço da história $\mathcal{M}$ como em (2.10). Notemos que esta é a "motivação" de definir o espaço da história dessa forma.

Consequentemente, o sistema correspondente à equação (1) se escreverá como:

$$
\begin{gathered}
\left|u_{t}\right|^{\rho} u_{t t}+A u_{t t}+A u+\int_{0}^{\infty} \mu(s) A \eta^{t}(s) d s+f(u)=h, \text { em } \Omega \times \mathbb{R}^{+} \\
\eta_{t}^{t}(s)=-\eta_{s}^{t}(s)+u_{t}, \quad \text { em } \quad \mathbb{R}^{+} \times \Omega \times \mathbb{R}^{+},
\end{gathered}
$$

com condiçoes de fronteira

$$
u=0 \text { sobre } \partial \Omega \times \mathbb{R}^{+}, \quad \eta=0 \text { sobre } \mathbb{R}^{+} \times \partial \Omega \times \mathbb{R}^{+}
$$

e dados iniciais

$$
u(x, 0)=u_{0}(x), \quad u_{t}(x, 0)=v_{0}(x), \quad \eta^{t}(x, 0)=0, \quad \eta^{0}(x, s)=\eta_{0}(x, s),
$$

onde

$$
\eta_{0}(x, s)=u_{0}(x, 0)-\phi(x, s), \quad(x, s) \in \Omega \times \mathbb{R}^{+} .
$$

Observação 2.12: Notemos que tendo em conta a equação (2.18), notamos que $\eta_{s}^{t} \in \mathcal{M}$, e ademais temos que $\eta^{t}(0)=0$ para cada $x \in \Omega$. Assim, pelo resultado (2.12) temos que $\eta^{t} \in \operatorname{dom}(T)$. Portanto nossa equação (2.18) também se pode escrever em termos de $T$ como:

$$
\eta_{t}^{t}(s)=T \eta^{t}(s)+u_{t}, \quad \text { em } \mathbb{R}^{+} \times \Omega \times \mathbb{R}^{+} .
$$

A seguir, vamos a apresentar nossos resultados para o sistema (2.17)-(2.18), que é autônomo. 


\section{Capítulo 3 \\ Boa colocação do problema}

Neste capitulo apresentaremos um dos resultados principais de nosso trabalho, a "boa colocação do problema". Começaremos definindo o que é uma solução fraca para nosso sistema e provaremos a existência de estas soluções pelo método de Faedo-Galerkind, método que é descrito de boa maneira no trabalho de Lions [19]. Para a prova de existência de soluções estudaremos os resultados apresentado por Cavalcanti, Domingos Cavalcanti \& Ferreira [6], Han \& Wang [17], Pata \& Zucchi [30] e faremos um estudo mais detalhado dos trabalhos de Conti, Marchini \& Pata [11] e Araujo, Ma \& Qin [2] para a prova da unicidade de soluções e a dependência continua dos dados.

Começaremos definindo o "Espaço de Fase" para nosso problema. Principalmente ele nos dirá onde moram as soluções fracas que vamos a definir.

Definição 3.1: (Espaço de Fase). Seja $\mathcal{M}$ como em (2.10). Definimos o "Espaço de Fase $\mathscr{H}^{\prime \prime}$ como

$$
\mathscr{H}:=H_{0}^{1}(\Omega) \times H_{0}^{1}(\Omega) \times \mathcal{M}
$$

o qual é um espaço normado, com a norma dada por:

$$
\|(u, v, \eta)\|_{\mathscr{H}}^{2}=\frac{1}{2}\|\nabla u\|_{2}^{2}+\frac{1}{2}\|\nabla v\|_{2}^{2}+\frac{1}{2}\|\eta\|_{\mathcal{M}}^{2}, \quad \forall(u, v, \eta) \in \mathscr{H} .
$$

Agora já temos todas as ferramenta para definir o que é uma solução fraca para o sistema (2.17)-(2.18).

Definição 3.2: Seja $\tau>0$ fixo mas arbitrário. Dado o sistema (2.17)-(2.18), com condições de fronteira (2.19) e dados iniciais (2.20). Se ditos dados iniciais $\left(u_{0}, v_{0}, \eta_{0}\right) \in \mathscr{H}$ e $h \in L^{2}(\Omega)$, então diremos que a função $z=\left(u, u_{t}, \eta\right) \in C([0, \tau], \mathscr{H})$ é uma "Soluçao Fraca" de (2.17)-(2.18) se satisfaz a condição inicial $z(0)=\left(u_{0}, v_{0}, \eta_{0}\right)$ e 


$$
\begin{gathered}
\left\langle\left|u_{t}\right|^{\rho} u_{t t}, \varphi\right\rangle+\left\langle u_{t t}, \varphi\right\rangle_{1}+\langle u, \varphi\rangle_{1}+\int_{0}^{\infty} \mu(s)\left\langle\eta^{t}(s), \varphi\right\rangle_{1} d s+\langle f(u), \varphi\rangle=(h, \varphi), \\
\left\langle\eta_{t}^{t}+\eta_{s}^{t}, \xi\right\rangle_{\mathcal{M}}=\left\langle u_{t}(t), \xi\right\rangle_{\mathcal{M}}
\end{gathered}
$$

para todo $\varphi \in H_{0}^{1}(\Omega), \xi \in \mathcal{M}$, e $t \in[0, \tau]$ quase sempre.

Observação 3.3: Uma pergunta obvia é, que sentido tem as expressões $\left\langle\left|u_{t}(t)\right|^{\rho} u_{t t}(t), \varphi\right\rangle$ e $\langle f(u(t)), \varphi\rangle$ na equação (3.1)?. Para responder isto analisaremos ambos casos.

- No caso, $\left\langle\left|u_{t}(t)\right|^{\rho} u_{t t}(t), \varphi\right\rangle$, dado que $u, u_{t} \in C\left([0, \tau], H_{0}^{1}(\Omega)\right)$ e $\rho \in[0,4)$, temos que

$$
\left|u_{t}\right|^{\rho} u_{t} \in L^{\infty}\left(0, \tau ; L^{\frac{\rho+2}{\rho+1}}(\Omega)\right)
$$

pois,

$$
\int_{\Omega}\left|u_{t}(t)\right|^{\frac{(\rho+2)(\rho+1)}{\rho+1}} d x=\left\|u_{t}(t)\right\|_{\rho+2}^{\rho+2} \leq C^{\rho+2}\left\|\nabla u_{t}(t)\right\|_{2}^{\rho+2}<\infty
$$

onde $C$ é a constante produto da imersão $H_{0}^{1}(\Omega) \hookrightarrow L^{\rho+2}(\Omega)$ a qual é valida dado que $\rho+2<6=2^{*}$.

Ademais se $\left\|\nabla u_{t}\right\|_{2} \leq R$ para algum $R>0$, se tem que $\left|u_{t}\right|^{\rho} u_{t}$ é uniformemente limitada em $L^{\frac{\rho+2}{\rho+1}}(\Omega)$ com respeito de $t$, pois

$$
\int_{0}^{\tau} \int_{\Omega}\left|u_{t}(t)\right|^{\frac{(\rho+2)(\rho+1)}{\rho+1}} d x d t=\int_{0}^{\tau}\left\|u_{t}(t)\right\|_{\rho+2}^{\rho+2} d t \leq C^{\rho+2} \int_{0}^{\tau}\left\|\nabla u_{t}(t)\right\|_{2}^{\rho+2} d t \leq \tau R^{\rho+2} C^{\rho+2} .
$$

Assim, é valida a seguinte igualdade no sentido das distribuições:

$$
\begin{aligned}
\left\langle\left|u_{t}(t)\right|^{\rho} u_{t t}(t), \varphi(t)\right\rangle_{D^{\prime}(\Omega) \times D(\Omega)} & =-\frac{1}{\rho+1}\left\langle\left|u_{t}(t)\right|^{\rho} u_{t}(t), \varphi_{t}(t)\right\rangle_{D^{\prime}(\Omega) \times D(\Omega)} \\
& =-\frac{1}{\rho+1} \int_{\Omega}\left|u_{t}(t)\right|^{\rho} u_{t}(t) \varphi_{t}(t) d x
\end{aligned}
$$

$\forall \varphi \in D([0, \tau], \Omega)$ e para quase todo $t \in[0, \tau]$, dado que $L^{\frac{\rho+2}{\rho+1}}(\Omega) \hookrightarrow L_{l o c}^{1}(\Omega) \hookrightarrow$ $D^{\prime}(\Omega)$ e da caracterização das distribuições que pertencem a $L_{l o c}^{1}(\Omega)$. Agora, como $\overline{D(\Omega)}^{H_{0}^{1}(\Omega)}=H_{0}^{1}(\Omega)$, por um passagem ao limite e pela continuidade podemos generalizar dita igualdade para todo $\varphi(t) \in H_{0}^{1}(\Omega)$, alem disso como $L^{\frac{\rho+2}{\rho+1}}(\Omega) \hookrightarrow H^{-1}(\Omega)$ pois $L^{\frac{\rho+2}{\rho+1}}(\Omega)=\left(L^{\rho+2}(\Omega)\right)^{\prime}$ a expressão $\left\langle\left|u_{t}(t)\right|^{\rho} u_{t t}(t), \varphi(t)\right\rangle$ se pode olhar como 
$\left\langle\left|u_{t}(t)\right|^{\rho} u_{t t}(t), \varphi(t)\right\rangle_{H^{-1}(\Omega) \times H_{0}^{1}(\Omega)}$ para todo $\varphi(t) \in H_{0}^{1}(\Omega)$ e para quase todo $t \in[0, \tau]$.

Então teremos que

$$
\begin{aligned}
\left\langle\left|u_{t}(t)\right|^{\rho} u_{t t}(t), \varphi(t)\right\rangle_{H^{-1}(\Omega) \times H_{0}^{1}(\Omega)} & =-\frac{1}{\rho+1}\left\langle\left|u_{t}(t)\right|^{\rho} u_{t}(t), \varphi_{t}(t)\right\rangle_{H^{-1}(\Omega) \times H_{0}^{1}(\Omega)} \\
& =-\frac{1}{\rho+1} \int_{\Omega}\left|u_{t}(t)\right|^{\rho} u_{t}(t) \varphi_{t}(t) d x
\end{aligned}
$$

- No caso, $\langle f(u(t)), \varphi\rangle$, procederemos praticamente igual ao casso anterior. Notemos que $f(u(t)) \in L^{\frac{q+2}{q+1}}(\Omega)$, para quase todo $t \in[0, \tau]$, pois: (omitiremos a variável $t$ nas contas, mas ter presente que o resultado é para quase todo $t \in[0, \tau])$

$$
\begin{aligned}
\int_{\Omega}|f(u)|^{\frac{q+2}{q+1}} d x & \leq \int_{\Omega}\left[c|u|\left(1+|u|^{q}\right)\right]^{\frac{q+2}{q+1}} d x \\
& \leq c^{\frac{q+2}{q+1}} 2^{\frac{q+2}{q+1}}\left[\int_{\Omega}|u|^{\frac{q+2}{q+1}} d x+\int_{\Omega}|u|^{q+2} d x\right] \\
& =c^{\frac{q+2}{q+1}} 2^{\frac{q+2}{q+1}}\|u\|_{\frac{q+2}{q+1}}^{\frac{q+2}{q+1}}+c^{\frac{q+2}{q+1}} 2^{\frac{q+2}{q+1}}\|u\|_{q+2}^{q+2} \\
& \leq\left(\frac{C_{f}^{1}}{2}\right)^{\frac{q+2}{q+1}}\|\nabla u\|_{2}^{\frac{q+2}{q+1}}+\left(\frac{C_{f}^{2}}{2}\right)^{\frac{q+2}{q+1}}\|\nabla u\|_{2}^{q+2}<\infty,
\end{aligned}
$$

onde se uso (2.2) e (2.1) na primeira desigualdade e as constantes $C_{f}^{1}$ e $C_{f}^{2}$ dependem de $c, q$ e as imersões $H_{0}^{1}(\Omega) \hookrightarrow L^{\frac{q+2}{q+1}}(\Omega)$ e $H_{0}^{1}(\Omega) \hookrightarrow L^{q+2}(\Omega)$ respectivamente dado que $q \in[0,4)$. Notemos ademais que

$\|f(u)\|_{\frac{q+2}{q+1}}=\left(\int_{\Omega}|f(u)|^{\frac{q+2}{q+1}} d x\right)^{\frac{q+1}{q+2}} \leq C_{f}^{1}\|\nabla u\|_{2}+C_{f}^{2}\|\nabla u\|_{2}^{q+1} \leq C_{f}\|\nabla u\|_{2}\left(1+\|\nabla u\|_{2}^{q}\right)$,

onde $C_{f}=\max \left\{C_{f}^{1}, C_{f}^{2}\right\}>0$.

Observação 3.4: Notemos que se $\left(u, u_{t}, \eta\right)$ é solução fraca do sistema (2.17)-(2.18) teremos que $\eta \in C([0, \tau], \mathcal{M})$ e $u_{t} \in L^{1}(0, \tau ; \mathcal{M})$. Então poderemos dizer que $\eta$ é uma solução suave para a equação (2.18), o mais claramente, de sua forma equivalente (2.21), isto é para

$$
\begin{gathered}
\frac{d \eta}{d t}=T \eta+u_{t} \\
\eta^{0}=\eta_{0} .
\end{gathered}
$$

Agora, como $\eta_{0} \in \mathcal{M}$, pela definição de solução suave, teremos que

$$
\eta^{t}=\Sigma(t) \eta_{0}+\int_{0}^{t} \Sigma(t-y) u_{t}(y) d y
$$

isto implica (por (2.11)) que a representação explicita para $\eta$ estará dada por

$$
\eta^{t}(s)=\left\{\begin{array}{lll}
u(t)-u(t-s) & \text { se } & 0<s \leq t \\
\eta_{0}(s-t)+u(t)-u_{0} & \text { se } & s>t
\end{array}\right.
$$

Notemos que esta ultima expressão é compatível com a construção do deslocamento $\eta^{t}$. 
Dado que já temos definido que é uma solução fraca para o sistema (2.17)-(2.18), começaremos a provar a existência de ditas soluções.

\subsection{Existência de soluções fracas}

Como se falo ao começo do capitulo, provaremos a existência de soluções fracas pelo método de Faedo-Galerkin. Começaremos enunciando o teorema que vai ser feito nesta seção.

\section{Teorema 3.5: (Existência de Soluções Fracas).}

Dado o sistema (2.17)-(2.18), com condições de fronteira (2.19) e dados iniciais (2.20). Suponha certa as hipóteses (H1) - (H4) . Se os dados iniciais $\left(u_{0}, v_{0}, \eta_{0}\right) \in \mathscr{H}$ e $h \in L^{2}(\Omega)$, então o sistema (2.17)-(2.18) possui uma solução fraca

$$
\left(u, u_{t}, \eta\right) \in C([0, \tau], \mathscr{H}), \quad \forall \tau>0
$$

satisfazendo

$$
\begin{gathered}
u \in L^{\infty}\left(\mathbb{R}^{+} ; H_{0}^{1}(\Omega)\right), \quad u_{t} \in L^{\infty}\left(\mathbb{R}^{+}, H_{0}^{1}(\Omega)\right), \\
u_{t t} \in L^{2}\left([0, \tau], H_{0}^{1}(\Omega)\right), \quad \eta \in L^{\infty}\left(\mathbb{R}^{+}, \mathcal{M}\right) .
\end{gathered}
$$

Para poder provar o teorema (3.5) começaremos fornecendo certas ferramentas que vai ajudar a aplicar o método de Faedo-Galerkin. Para maior detalhe se remite ao leitor revisar os trabalhos de Conti, Marchini \& Para [11], Araujo, Ma \& Qin [2], Cavalcanti, Domingos Cavalcanti \& Ferreira [6], Han \& Wang [17] e Pata \& Zucchi [30].

Como sabemos o método de Faedo-Galerkin consiste em ter uma base de auto-funções "boa" para modelar o problema aproximado referente à equação e poder trabalhar em ele. Começaremos fornecendo dita base.

Seja $\left\{\omega_{j}\right\}_{j=1}^{\infty}$ a base de auto-funções de $-\Delta u=\lambda u$ em $\Omega$, com condições de fronteira de Dirichlet. Então, dita base é ortonormal em $L^{2}(\Omega)$ e ortogonal em $H_{0}^{1}(\Omega)$. Ademais cumpre que:

$$
\left\{\begin{aligned}
-\Delta \omega_{j} & =\lambda_{j} \omega_{j}, & & x \in \Omega \\
\omega_{j} & =0, & & x \in \partial \Omega
\end{aligned}\right.
$$

onde $\lambda_{j} \geq 0, \forall j \in \mathbb{N}$ e $\lambda_{i} \neq \lambda_{j}$ para $i \neq j$. 
Observação 3.6: Notemos de dita base de auto-funções existe, pois podemos olhar $-\Delta=A$, onde $A: \operatorname{dom}(A) \subset L^{2}(\Omega) \rightarrow H_{0}^{1}(\Omega)$ com $H_{0}^{1}(\Omega) \stackrel{c}{\hookrightarrow} L^{2}(\Omega)$, e olhando o produto interno em $H_{0}^{1}(\Omega)$ como uma forma bilinear continua, cumprindo que $(A u, v)=\langle u, v\rangle$ temos que $A$ é fechado, não limitado, positivo definido, autoadjunto e é um isomorfismo (vide Temam [36] Capítulo 2). Assim, da teoria espectral, existe a base $\left\{\omega_{j}\right\}_{j=1}^{\infty}$.

Assim, já temos uma base para $L^{2}(\Omega)$ e $H_{0}^{1}(\Omega)$. Agora temos que construir uma base para $\mathcal{M}$.

Lema 3.7: Existe uma base $\left\{\zeta_{j}\right\}_{j=1}^{\infty}$ ortonormal para $\mathcal{M}=L_{\mu}^{2}\left(\mathbb{R}^{+} ; H_{0}^{1}(\Omega)\right)$ com $\zeta_{j} \in$ $C_{0}^{\infty}\left(\mathbb{R}^{+} ; H_{0}^{1}(\Omega)\right)$, para todo $j \in \mathbb{N}$.

Demonstração. Sejam $\left\{h_{j}\right\}_{j=1}^{\infty}$ uma base ortonormal de $L_{\mu}^{2}\left(\mathbb{R}^{+}\right) \cap C_{0}^{\infty}\left(\mathbb{R}^{+}\right)$e $\left\{\bar{\omega}_{j}\right\}_{j=1}^{\infty}$ tal que

$$
\bar{\omega}_{j}=\frac{\omega_{j}}{\left\|\nabla \omega_{j}\right\|_{2}}, \quad \forall j \in \mathbb{N} .
$$

Definindo $\zeta_{i}=h_{p} \bar{\omega}_{k}$ e $\zeta_{j}=h_{m} \bar{\omega}_{n}$, obtemos que:

$$
\begin{aligned}
\left\langle\zeta_{i}, \zeta_{j}\right\rangle_{\mathcal{M}} & =\int_{0}^{\infty} \mu(s)\left(\int_{\Omega} \nabla \zeta_{i} \nabla \zeta_{j} d x\right) d s \\
& =\int_{0}^{\infty} \mu(s) h_{p}(s) h_{m}(s)\left(\int_{\Omega} \nabla \bar{\omega}_{k} \nabla \bar{\omega}_{n} d x\right) d s \\
& =\delta_{p m} \delta_{k n} \\
& =\delta_{i j},
\end{aligned}
$$

Assim, $\left\{\zeta_{j}\right\}_{j=1}^{\infty}$ é uma base ortonormal para $\mathcal{M} \operatorname{com} \zeta_{j} \in C_{0}^{\infty}\left(\mathbb{R}^{+} ; H_{0}^{1}(\Omega)\right), \forall j \in \mathbb{N}$.

Dado que já temos definidas as respectivas bases ortonormais para nossos espaços, podemos começar a prova do Teorema 3.5.

Demonstração. (Teorema 3.5). A prova sera feita em cinco etapas, começaremos definindo o "Problema Aproximado" para o sistema (2.17)-(2.18). Logo faremos três tipos diferentes de estimativas a partir de dito problema aproximado e finalmente procederemos a explicar em que "sentido" se aplicara o limite no problema.

\subsubsection{Problema aproximado}

Para cada $m \in \mathbb{N}$, consideremos os subespaços gerados

$$
\operatorname{Span}\left\{\omega_{1}, \ldots, \omega_{m}\right\} \subset H_{0}^{1}(\Omega) \quad \text { e } \operatorname{Span}\left\{\zeta_{1}, \ldots, \zeta_{m}\right\} \subset \mathcal{M}
$$


Para os dados iniciais $\left(u_{0}, v_{0}, \eta_{0}\right) \in \mathscr{H}$, procuramos as soluções aproximadas

$$
u^{m}(t)=\sum_{j=1}^{m} a_{m j}(t) \omega_{j} \quad \text { e } \quad \eta^{t, m}=\sum_{j=1}^{m} b_{m j}(t) \zeta_{j}(s)
$$

tais que

$$
\begin{gathered}
\left\langle\left|u_{t}^{m}\right|^{\rho} u_{t t}^{m}, \omega_{j}\right\rangle+\left\langle u_{t t}^{m}, \omega_{j}\right\rangle_{1}+\left\langle u^{m}, \omega_{j}\right\rangle_{1}+\int_{0}^{\infty} \mu(s)\left\langle\eta^{t, m}(s), \omega_{j}\right\rangle_{1} d s+\left\langle f\left(u^{m}\right), \omega_{j}\right\rangle=\left(h, \omega_{j}\right), \\
\left\langle\eta_{t}^{t, m}, \zeta_{j}\right\rangle_{\mathcal{M}}=-\left\langle\eta_{s}^{t, m}, \zeta_{j}\right\rangle_{\mathcal{M}}+\left\langle u_{t}^{m}(t), \zeta_{j}\right\rangle_{\mathcal{M}},
\end{gathered}
$$

para $j \in[1, m]$, com condições iniciais

$$
u^{m}(0)=u_{0}^{m}, \quad u_{t}^{m}(0)=v_{0}^{m}, \quad \eta^{0, m}=\eta_{0}^{m},
$$

onde $u_{0}^{m}, v_{0}^{m}, \eta_{0}^{m}$ são escolhidos de forma que

$$
u_{0}^{m} \rightarrow u_{0} \text { em } H_{0}^{1}(\Omega), \quad v_{0}^{m} \rightarrow v_{0} \text { em } H_{0}^{1}(\Omega), \quad \eta_{0}^{m} \rightarrow \eta_{0} \text { em } \mathcal{M}
$$

Assim, o sistema (3.5)-(3.6) com condições iniciais (3.7) é um sistema de equações diferenciais ordinárias em $t$. Tal sistema possui existência local via Teorema de Carathéodory (vide Han \& Wang [17]). Agora, faremos certas estimativas a priori que mostrarão que as soluções locais $\left(u^{m}, u_{t}^{m}, \eta^{t, m}\right)$ podem ser estendidas ao intervalo $[0, \infty)$.

\subsubsection{Primeira estimativa}

Dado que temos que:

$$
u_{t}^{m}(t)=\sum_{j=1}^{m} a_{m j}^{\prime}(t) \omega_{j} \quad \text { e } \quad \eta^{t, m}=\sum_{j=1}^{m} b_{m j}(t) \zeta_{j}(s),
$$

Então, multiplicamos por $a_{m j}^{\prime}(t)$ à equação $(3.5)$ e por $b_{m j}(t)$ à equação (3.6), tomando a somatória em ambas equações desde $j=1$ até $j=m$, obtemos

$$
\begin{gathered}
\left\langle\left|u_{t}^{m}\right|^{\rho} u_{t t}^{m}, u_{t}^{m}\right\rangle+\left\langle u_{t t}^{m}, u_{t}^{m}\right\rangle_{1}+\left\langle u^{m}, u_{t}^{m}\right\rangle_{1}+\int_{0}^{\infty} \mu(s)\left\langle\eta^{t, m}(s), u_{t}^{m}\right\rangle_{1} d s+\left\langle f\left(u^{m}\right), u_{t}^{m}\right\rangle=\left(h, u_{t}^{m}\right), \\
\left\langle\eta_{t}^{t, m}, \eta^{t, m}\right\rangle_{\mathcal{M}}=-\left\langle\eta_{s}^{t, m}, \eta^{t, m}\right\rangle_{\mathcal{M}}+\left\langle u_{t}^{m}(t), \eta^{t, m}\right\rangle_{\mathcal{M}}
\end{gathered}
$$


Definição 3.8: Definimos o funcional

$$
\mathcal{E}(t):=\frac{1}{2}\|\nabla u(t)\|_{2}^{2}+\frac{1}{2}\left\|\nabla u_{t}(t)\right\|_{2}^{2}+\frac{1}{2}\left\|\eta^{t}\right\|_{\mathcal{M}}^{2}=\left\|\left(u(t), u_{t}(t), \eta^{t}\right)\right\|_{\mathscr{H}},
$$

o qual sera chamado por "Energia Auxiliar do Sistema (3.9)-(3.10)".

Em particular, tendo em conta o problema aproximado anterior, denotaremos por

$$
\mathcal{E}^{m}(t):=\frac{1}{2}\left\|\nabla u^{m}(t)\right\|_{2}^{2}+\frac{1}{2}\left\|\nabla u_{t}^{m}(t)\right\|_{2}^{2}+\frac{1}{2}\left\|\eta^{t, m}\right\|_{\mathcal{M}}^{2}=\left\|\left(u^{m}(t), u_{t}^{m}(t), \eta^{t, m}\right)\right\|{ }_{\mathscr{C}} .
$$

Definição 3.9: Definimos o funcional

$$
E(t)=\frac{1}{\rho+2}\left\|u_{t}(t)\right\|_{\rho+2}^{\rho+2}+\mathcal{E}(t)+\int_{\Omega} \widehat{f}(u(t)) d x-\int_{\Omega} h u(t) d x
$$

ou seja,

$$
E(t)=\frac{1}{\rho+2}\left\|u_{t}(t)\right\|_{\rho+2}^{\rho+2}+\frac{1}{2}\|\nabla u(t)\|_{2}^{2}+\frac{1}{2}\left\|\nabla u_{t}(t)\right\|_{2}^{2}+\frac{1}{2}\left\|\eta^{t}\right\|_{\mathcal{M}}^{2}+\int_{\Omega} \widehat{f}(u(t)) d x-\int_{\Omega} h u(t) d x .
$$

Dito funcional sera chamado de "Energia do Sistema (3.9)-(3.10)".

Em particular, tendo em conta o problema aproximado anterior, denotaremos por

$$
E^{m}(t):=\frac{1}{\rho+2}\left\|\nabla u_{t}^{m}(t)\right\|_{\rho+2}^{\rho+2}+\mathcal{E}^{m}(t)+\int_{\Omega} \widehat{f}\left(u^{m}(t)\right) d x-\int_{\Omega} h u^{m}(t) d x .
$$

O qual chamaremos por "Energia Aproximada"para o sistema (3.9)-(3.10).

Agora notemos que

$$
\begin{aligned}
\frac{1}{\rho+2} \frac{d}{d t}\left\|u_{t}^{m}(t)\right\|_{\rho+2}^{\rho+2} & =\left\langle\left|u_{t}^{m}(t)\right|^{\rho} u_{t t}^{m}(t), u_{t}^{m}(t)\right\rangle, \\
\frac{1}{2} \frac{d}{d t}\left\|\nabla u^{m}(t)\right\|_{2}^{2} & =\left\langle u^{m}(t), u_{t}^{m}(t)\right\rangle_{1}, \\
\frac{1}{2} \frac{d}{d t}\left\|\nabla u_{t}^{m}(t)\right\|_{2}^{2} & =\left\langle u_{t t}^{m}(t), u_{t}^{m}(t)\right\rangle_{1}, \\
\frac{1}{2} \frac{d}{d t}\left\|\eta^{t, m}\right\|_{\mathcal{M}}^{2} & =\left\langle\eta_{t}^{t, m}, \eta^{t, m}\right\rangle_{\mathcal{M}}, \\
\frac{d}{d t} \int_{\Omega} \widehat{f}\left(u^{m}(t)\right) d x & =\left\langle f\left(u^{m}(t)\right), u_{t}^{m}(t)\right\rangle, \\
\frac{d}{d t} \int_{\Omega} h u^{m}(t) d x & =\left(h, u_{t}^{m}(t)\right),
\end{aligned}
$$

assim, temos de estas igualdades, da definição de $E^{m}(t)$ e das equações (3.9) e (3.10), que

$$
\frac{d}{d t} E^{m}(t)=-\left\langle\eta_{s}^{t, m}, \eta^{t, m}\right\rangle_{\mathcal{M}}=\left\langle T \eta^{t, m}, \eta^{t, m}\right\rangle_{\mathcal{M}} \leq 0
$$


Esto ultimo é pela desigualdade (2.13).

Agora, integrando de 0 a $t$ a desigualdade acima, obtemos

$$
E^{m}(t) \leq E^{m}(0)
$$

mas, esta desigualdade se pode melhorar a partir dos dados iniciais, para isto analisemos $E^{m}(0)$, então temos que

$$
E^{m}(0)=\frac{1}{\rho+2}\left\|u_{t}^{m}(0)\right\|_{\rho+2}^{\rho+2}+\mathcal{E}^{m}(0)+\int_{\Omega} \widehat{f}\left(u^{m}(0)\right) d x-\int_{\Omega} h u^{m}(0) d x,
$$

de onde observamos que:

- Suponhamos que $\left\|\left(u_{0}, v_{0}, \eta_{0}\right)\right\|_{\mathscr{H}}^{2} \leq R$, então como

$$
\mathcal{E}^{m}(0)=\left\|\left(u^{m}(0), u_{t}^{m}(0), \eta^{t, m}(0)\right)\right\|_{\mathscr{H}}^{2}=\left\|\left(u_{0}^{m}, v_{0}^{m}, \eta_{0}^{m}\right)\right\|_{\mathscr{H}}^{2},
$$

e pelas convergências (3.8), temos que $\mathcal{E}^{m}(0) \leq Q_{1}(R)$ onde $Q_{1}: \mathbb{R}^{+} \rightarrow \mathbb{R}^{+}$é uma função crescente e independente do tempo.

- Suponhamos que $\left\|\left(u_{0}, v_{0}, \eta_{0}\right)\right\|_{\mathscr{H}}^{2} \leq R$, então

$$
\frac{1}{\rho+2}\left\|u_{t}^{m}(0)\right\|_{\rho+2}^{\rho+2} \leq \frac{1}{\rho+2} C^{\rho+2}\left\|\nabla u_{0}^{m}\right\|_{2}^{\rho+2} \leq \frac{1}{\rho+2} C^{\rho+2} R^{\frac{\rho+2}{2}}:=Q_{2}(R),
$$

onde a segunda desigualdade é dada pela imersão $H_{0}^{1}(\Omega) \hookrightarrow L^{\rho+2}(\Omega)$ com constante $C>0$. Ademais, análogo a $Q_{1}, Q_{2}$ é uma função crescente e independente do tempo.

- Suponhamos que $\left\|\left(u_{0}, v_{0}, \eta_{0}\right)\right\|_{\mathscr{H}}^{2} \leq R$, então

$$
\left.\int_{\Omega} \widehat{f}\left(u^{m}(0)\right) d x=\int_{\Omega} \widehat{f}\left(u_{0}^{m}\right)\right) d x
$$

pela hipóteses $(2.4),(2.2)$ e (2.1) temos que

$\widehat{f}(u) \leq f(u) u+\frac{\beta}{2} u^{2}+m_{f} \leq c|u|\left(1+|u|^{q}\right) u+\frac{\beta}{2} u^{2}+m_{f} \leq c\left(|u|^{2}+|u|^{q+2}\right)+\frac{\beta}{2}|u|^{2}+m_{f}$,

assim, existira uma função $Q_{3}$ análoga as anteriores tal que

$$
\int_{\Omega} \widehat{f}\left(u^{m}(0)\right) d x \leq Q_{3}(R)
$$

- Suponhamos que $\left\|\left(u_{0}, v_{0}, \eta_{0}\right)\right\|_{\mathscr{H}}^{2} \leq R$, então

$$
\int_{\Omega} h u^{m}(0) d x \leq\|h\|_{2}\left\|u_{0}^{m}\right\|_{2} \leq \epsilon\|h\|_{2}^{2}+\frac{1}{4 \epsilon}\left\|u_{0}^{m}\right\|_{2}^{2} \leq \epsilon\|h\|_{2}^{2}+\frac{1}{4 \lambda_{1} \epsilon}\left\|\nabla u_{0}^{m}\right\|_{2}^{2}=\frac{1}{\lambda_{1}}\|h\|_{2}^{2}+\frac{1}{4}\left\|\nabla u_{0}^{m}\right\|_{2}^{2}
$$


onde a segunda desigualdade se obtêm usando a desigualdade de Young $\operatorname{com} \epsilon$, a terceira desigualdade é dada pela imersão $H_{0}^{1}(\Omega) \hookrightarrow L^{2}(\Omega)$ com constante de imersão $\lambda_{1}>0$ e para ter a igualdade final só tomamos $\epsilon=\frac{1}{\lambda_{1}}$. Análogo aos outros casos, existe uma função $Q_{4}$ tal que

$$
\int_{\Omega} h u^{m}(0) d x \leq Q_{4}(R)
$$

Assim, podemos dizer que se $\left\|\left(u_{0}, v_{0}, \eta_{0}\right)\right\|_{\mathscr{H}}^{2} \leq R$, existe uma função $Q_{0}: \mathbb{R}^{+} \rightarrow \mathbb{R}^{+}$ crescente e independente do tempo, tal que

$$
E^{m}(0) \leq Q(R)
$$

Observação 3.10: Notemos que $Q_{0}(R)$, não só depende dos dados iniciais, também depende de $\|h\|, m_{f}, \beta, \rho, q,|\Omega|, \lambda_{1}$ e algumas constantes de imersões antes mencionadas.

Agora encontremos alguma relação entre $\mathcal{E}^{m}(t)$ e $E^{m}(0)$.

Notemos que como $\frac{1}{\rho+2}\left\|u_{t}^{m}(t)\right\|_{\rho+2}^{\rho+2} \geq 0$ temos

$$
E^{m}(t) \geq \mathcal{E}^{m}(t)+\int_{\Omega} \widehat{f}\left(u^{m}(t)\right) d x-\int_{\Omega} h u^{m}(t) d x
$$

ademais procedendo de maneira análoga como em (3.15) temos que

$$
-\int_{\Omega} h u^{m}(t) d x \geq-\frac{1}{\lambda_{1}}\|h\|_{2}^{2}-\frac{1}{4}\left\|\nabla u^{m}(t)\right\|_{2}^{2}
$$

assim, obtemos que

$$
E^{m}(t) \geq \frac{1}{2} \mathcal{E}^{m}(t)+\int_{\Omega} \widehat{f}\left(u^{m}(t)\right) d x-\frac{1}{\lambda_{1}}\|h\|_{2}^{2}
$$

Notemos agora que pela hipóteses (2.3), dado $\epsilon>0$ (pequeno) existe $R>0$ tal que

$$
\frac{f(y)}{y}>-\lambda_{1}+\epsilon \quad \text { se }|y|>R
$$

logo, seja $\lambda=\lambda_{1}-\epsilon>0$ para $\epsilon$ o suficientemente pequeno, então, existe $C>0$ tal que

$$
f(y) \geq-\lambda y-C,
$$

notemos que esta $C$ vem dos dados referentes a $0 \leq|y| \leq R$. Integrando de 0 a $u$ respeito a $y$, teremos que

$$
\widehat{f}(u) \geq-\frac{\lambda}{2} u^{2}-C u,
$$


agora integrando sobre $\Omega$ teremos que

$$
\int_{\Omega} \widehat{f}(u) d x \geq-\frac{\lambda}{2}\|u\|_{2}^{2}-C\|u\|_{1}
$$

então, dada a imersão $L^{2}(\Omega) \hookrightarrow L^{1}(\Omega)$, existira uma constante $\bar{\lambda}>0$ que depende de $\lambda$ e a constante de imersão, tal que

$$
\int_{\Omega} \widehat{f}(u) d x \geq-\bar{\lambda}\|u\|_{2}^{2}
$$

finalmente dada a imersão $H_{0}^{1}(\Omega) \hookrightarrow L^{2}(\Omega)$, teremos que

$$
\int_{\Omega} \widehat{f}(u) d x \geq-\frac{\bar{\lambda}}{\lambda_{1}}\|\nabla u\|_{2}^{2} \geq-\frac{2 \bar{\lambda}}{\lambda_{1}} \mathcal{E}(t) .
$$

Assim, tomando $\epsilon>0$ muito pequeno, existira uma constante fixa $\nu>0$ tal que

$$
E^{m}(t) \geq \nu \mathcal{E}^{m}(t)-\frac{1}{\lambda_{1}}\|h\|_{2}^{2}
$$

ou seja,

$$
\mathcal{E}^{m}(t) \leq \frac{E^{m}(t)+C_{h}}{\nu}
$$

onde $C_{h}=\frac{1}{\lambda_{1}}\|h\|_{2}^{2}$.

Com isto, e por (3.14) e (3.16), se $\left\|\left(u_{0}, v_{0}, \eta_{0}\right)\right\|_{\mathscr{H}}^{2} \leq R$, existe uma função $Q: \mathbb{R}^{+} \rightarrow \mathbb{R}^{+}$ crescente e independente do tempo, tal que

$$
\mathcal{E}^{m}(t) \leq Q(R)
$$

Com a estimativa acima concluímos que

$$
\begin{gathered}
u^{m}(t) \text { é limitado em } L^{\infty}\left(\mathbb{R}^{+} ; H_{0}^{1}(\Omega)\right), \\
u_{t}^{m}(t) \text { é limitado em } L^{\infty}\left(\mathbb{R}^{+} ; H_{0}^{1}(\Omega)\right), \\
\eta^{t, m} \text { é limitado em } L^{\infty}\left(\mathbb{R}^{+} ; \mathcal{M}\right) .
\end{gathered}
$$

isto é, se tem as seguintes convergências fraca estrela

$$
\begin{gathered}
u^{m} \stackrel{*}{\rightarrow} u \text { em } L^{\infty}\left(\mathbb{R}^{+} ; H_{0}^{1}(\Omega)\right), \\
u_{t}^{m} \stackrel{*}{\rightarrow} u_{t} \quad \text { em } L^{\infty}\left(\mathbb{R}^{+} ; H_{0}^{1}(\Omega)\right), \\
\eta^{\bullet}, m \stackrel{*}{\rightarrow} \eta^{\bullet} \quad \text { em } L^{\infty}\left(\mathbb{R}^{+} ; \mathcal{M}\right) .
\end{gathered}
$$

Observação 3.11: A expressão $\eta^{\bullet, m}$ denota à função $\eta^{\bullet, m}: \mathbb{R}^{+} \rightarrow \mathcal{M}$ tal que $\eta^{\bullet, m}(t)=$ $\eta^{t, m}$. Análogo para $\eta^{\bullet}$. Esta notação se usara ao largo do trabalho cada vez que se precise. 


\subsubsection{Segunda estimativa}

Nesta subseção queremos estimar principalmente $\int_{0}^{t}\left\|\nabla u_{t t}^{m}(s)\right\|_{2}^{2} d s$ para $t \in[0, \tau] \operatorname{com}$ $\tau>0$ fixo.

Começaremos multiplicando a equação (3.5) por $a_{m j}^{\prime \prime}$ e somando em relação a $j$ de 1 até $m$, assim obtemos

$\left\langle\left|u_{t}^{m}\right|^{\rho} u_{t t}^{m}, u_{t t}^{m}\right\rangle+\left\|\nabla u_{t t}^{m}\right\|_{2}^{2}=-\left\langle u^{m}, u_{t t}^{m}\right\rangle_{1}-\int_{0}^{\infty} \mu(s)\left\langle\eta^{t, m}(s), u_{t t}^{m}\right\rangle_{1} d s-\left\langle f\left(u^{m}\right), u_{t t}^{m}\right\rangle+\left(h, u_{t t}^{m}\right)$,

dado que $\left\langle\left|u_{t}^{m}\right|^{\rho} u_{t t}^{m}, u_{t t}^{m}\right\rangle \geq 0$, teremos que

$$
\left\|\nabla u_{t t}^{m}\right\|_{2}^{2} \leq-\left\langle u^{m}, u_{t t}^{m}\right\rangle_{1}-\int_{0}^{\infty} \mu(s)\left\langle\eta^{t, m}(s), u_{t t}^{m}\right\rangle_{1} d s-\left\langle f\left(u^{m}\right), u_{t t}^{m}\right\rangle+\left(h, u_{t t}^{m}\right) .
$$

Notemos que para estimar $\left\|\nabla u_{t t}^{m}\right\|_{2}^{2}$, basta estimar cada termo do lado direito da igualdade acima.

- Estimando $\left\langle u^{m}, u_{t t}^{m}\right\rangle_{1}$

$$
\left\langle u^{m}, u_{t t}^{m}\right\rangle_{1} \leq\left\|\nabla u^{m}\right\|_{2}\left\|\nabla u_{t t}^{m}\right\|_{2} .
$$

- Estimando $\int_{0}^{\infty} \mu(s)\left\langle\eta^{t, m}(s), u_{t t}^{m}\right\rangle_{1} d s$.

$$
\begin{aligned}
\int_{0}^{\infty} \mu(s)\left\langle\eta^{t, m}(s), u_{t t}^{m}\right\rangle_{1} d s & =\int_{0}^{\infty} \mu(s)\left[\int_{\Omega} \nabla \eta^{t, m}(s) \nabla u_{t t}^{m} d x\right] d s \\
& \leq \int_{0}^{\infty} \mu(s)\left\|\nabla \eta^{t, m}(s)\right\|_{2}\left\|\nabla u_{t t}^{m}\right\|_{2} d s \\
& =\left\|\nabla u_{t t}^{m}\right\|_{2}\left(\int_{0}^{\infty} \mu(s)\left\|\nabla \eta^{t, m}(s)\right\|_{2}\right) d s \\
& =\left\|\nabla u_{t t}^{m}\right\|_{2}\left(\int_{0}^{\infty} \mu^{1 / 2} \mu^{1 / 2}\left\|\nabla \eta^{t, m}(s)\right\|_{2} d s\right) \\
& \leq\left\|\nabla u_{t t}^{m}\right\|_{2}\left(\int_{0}^{\infty} \mu(s) d s\right)^{1 / 2}\left(\int_{0}^{\infty} \mu(s)\left\|\nabla \eta^{t, m}(s)\right\|_{2} d s\right)^{1 / 2} \\
& =k_{0}^{1 / 2}\left\|\nabla u_{t t}^{m}\right\|_{2}\left\|\eta^{t, m}\right\|_{\mathcal{M} .}
\end{aligned}
$$

Assim

$$
\int_{0}^{\infty} \mu(s)\left\langle\eta^{t, m}(s), u_{t t}^{m}\right\rangle_{1} d s \leq k_{0}^{1 / 2}\left\|\nabla u_{t t}^{m}\right\|_{2}\left\|\eta^{t, m}\right\|_{\mathcal{M}}
$$

- Estimando $\left\langle f\left(u^{m}\right), u_{t t}^{m}\right\rangle$

Pela observação (3.3) temos que

$$
\left\langle f\left(u^{m}\right), u_{t t}^{m}\right\rangle \leq\left\|f\left(u^{m}\right)\right\|_{\frac{q+2}{q+1}}\left\|u_{t t}^{m}\right\|_{q+2} \leq C\left\|\nabla u_{t t}^{m}\right\|_{2}\left(C_{f}^{1}\left\|\nabla u^{m}\right\|_{2}+C_{f}^{2}\left\|\nabla u^{m}\right\|_{2}^{q+1}\right),
$$

onde $C>0$ é a constante da imersão $H_{0}^{1}(\Omega) \hookrightarrow L^{q+2}(\Omega)$, pois lembremos que $q \in[0,4)$. 
- Estimando $\left(h, u_{t t}^{m}\right)$

$$
\left(h, u_{t t}^{m}\right) \leq\|h\|_{2}\left\|u_{t t}^{m}\right\|_{2} \leq \frac{1}{\lambda_{1}}\|h\|_{2}\left\|\nabla u_{t t}^{m}\right\|_{2} .
$$

Assim, a partir de estas estimativas teremos que

$$
\left\|\nabla u_{t t}^{m}\right\|_{2}^{2} \leq\left\|\nabla u_{t t}^{m}\right\|_{2}\left(\left\|\nabla u^{m}\right\|_{2}+k_{0}^{1 / 2}\left\|\eta^{t, m}\right\|_{\mathcal{M}}+C C_{f}^{1}\left\|\nabla u^{m}\right\|_{2}+C C_{f}^{2}\left\|\nabla u^{m}\right\|_{2}^{q+1}+\frac{1}{\lambda_{1}}\|h\|_{2}\right) .
$$

Analogamente como se trabalho na primeira estimativa, se $\left\|\left(u_{0}, v_{0}, \eta_{0}\right)\right\|_{\mathscr{H}}^{2} \leq R$, existe uma função $Q: \mathbb{R}^{+} \rightarrow \mathbb{R}^{+}$crescente e independente do tempo, tal que

$$
\left\|\nabla u_{t t}^{m}\right\|_{2}^{2} \leq \frac{1}{2}\left\|\nabla u_{t t}^{m}\right\|_{2}^{2}+\frac{1}{2} Q(R)
$$

então,

$$
\left\|\nabla u_{t t}^{m}\right\|_{2}^{2} \leq Q(R)
$$

Agora, seja $\tau>0$ fixo com $t \in[0, \tau]$. Integrando de 0 a $t$ temos

$$
\int_{0}^{t}\left\|\nabla u_{t t}^{m}(s)\right\|_{2}^{2} d s \leq t Q(R) \leq \tau Q(R), \quad t \in[0, \tau]
$$

Notemos que a cota superior $\tau Q(R)$ agora não só depende dos dados iniciais, se não que agora também depende de $\tau$.

Com a estimativa acima concluímos que

$$
\left(u_{t t}^{m}\right) \text { é limitada em } L^{2}\left(0, \tau ; H_{0}^{1}(\Omega)\right)
$$

ou seja, se tem a seguinte convergência fraca estrela

$$
u_{t t}^{m} \stackrel{*}{\rightarrow} u_{t t} \text { em } L^{2}\left(0, \tau ; H_{0}^{1}(\Omega)\right) .
$$

\subsubsection{Terceira estimativa}

Nesta subseção queremos estimar $\left\|\left|u_{t}^{m}(t)\right|^{\rho} u_{t}^{m}(t)\right\|_{\frac{\rho+2}{\rho+1}}$. Esta estimativa junto com a estimativa anterior, nos dará a convergência para o termo não linear $\left|u_{t}^{m}(t)\right|^{\rho} u_{t t}^{m}(t)$. Assim, teremos que

$$
\left\|\left|u_{t}^{m}(t)\right|^{\rho} u_{t}^{m}(t)\right\|_{\frac{\rho+2}{\rho+1}}=\left\|u_{t}^{m}(t)\right\|_{\rho+2}^{\rho+1} \leq C^{\rho+1}\left\|\nabla u_{t}^{m}(t)\right\|_{2}^{\rho+1}
$$

onde $C>0$ é uma constante que se obtêm da imersão $H_{0}^{1}(\Omega) \hookrightarrow L^{\rho+2}(\Omega)$.

Procedendo igual que a segunda estimativa, teremos que para algum $\tau>0$ fixo se cumpre que

$$
\left(\left|u_{t}^{m}\right|^{\rho} u_{t}^{m}\right) \text { é limitada em } L^{\infty}\left(0, \tau ; L^{\frac{\rho+2}{\rho+1}}(\Omega)\right)
$$




\subsubsection{Passagem ao limite}

Com as estimativas a priori obtidas acima, podemos passar ao limite as soluções aproximadas e garantir a existência de uma solução global fraca satisfazendo os dados iniciais, mas o problema acontece com os termos não lineares, pelo qual nesta subseção estudaremos "em que sentido" sera o passagem ao limite para ditos termos. Os termos não lineares na equação são

$$
f\left(u^{m}\right) \text { e }\left|u_{t}^{m}\right|^{\rho} u_{t t}^{m}
$$

Alem disso, será feito um estudo do passagem ao limite para o problema aproximado da equação (2.18).

- Limite para $\left|u_{t}^{m}\right|^{\rho} u_{t t}^{m}$.

Como olhamos na observação (3.3), para cada $t \in[0, \tau]$, temos que $\left|u_{t}^{m}(t)\right|^{\rho} u_{t}^{m}(t) \in$ $L^{\frac{\rho+2}{\rho+1}}(\Omega)$. Pela imersão compacta $W^{1, \infty}\left(0, \tau ; H_{0}^{1}(\Omega)\right) \stackrel{c}{\hookrightarrow} C\left([0, \tau] ; L^{2}(\Omega)\right)$, passando a uma subsequencia se for necessário, teremos que

$$
u_{t}^{m} \rightarrow u_{t} \quad \text { em } C\left([0, \tau] ; L^{2}(\Omega)\right),
$$

isto é pela primeira e segunda estimativa. Assim, em particular, teremos que

$$
\left|u_{t}^{m}\right|^{\rho} u_{t}^{m} \rightarrow\left|u_{t}\right|^{\rho} u_{t} \text { q.s. em } \Omega \times[0, \tau]
$$

Alem disso, pela terceira estimativa temos que

$$
\left(\left|u_{t}^{m}\right|^{\rho} u_{t}^{m}\right) \text { é limitada em } L^{\infty}\left(0, \tau ; L^{\frac{\rho+2}{\rho+1}}(\Omega)\right)
$$

ou seja $\left|u_{t}^{m}\right|^{\rho} u_{t}^{m}$ é uniformemente limitada em $L^{\frac{\rho+2}{\rho+1}}(\Omega)$ respeito de $t \in[0, \tau]$. Assim, pelo teorema de convergência dominada e pela definição de derivada distribucional, teremos que

$$
-\frac{1}{p+1} \int_{0}^{\tau}\left\langle\left|u_{t}^{m}(t)\right|^{\rho} u_{t}^{m}(t), \varphi_{t}(t)\right\rangle d t \rightarrow \int_{0}^{\tau}\left\langle\left|u_{t}(t)\right|^{\rho} u_{t t}(t), \varphi(t)\right\rangle d t,
$$

onde $\varphi \in D\left([0, \tau] ; H_{0}^{1}(\Omega)\right)$. Agora, por outro lado, temos que

$$
-\frac{1}{p+1} \int_{0}^{\tau}\left\langle\left|u_{t}^{m}(t)\right|^{\rho} u_{t}^{m}(t), \varphi_{t}(t)\right\rangle d t=\int_{0}^{\tau}\left\langle\left|u_{t}^{m}(t)\right|^{\rho} u_{t t}^{m}(t), \varphi(t)\right\rangle d t
$$

então

$$
\int_{0}^{\tau}\left\langle\left|u_{t}^{m}(t)\right|^{\rho} u_{t t}^{m}(t), \varphi(t)\right\rangle d t \rightarrow \int_{0}^{\tau}\left\langle\left|u_{t}(t)\right|^{\rho} u_{t t}(t), \varphi(t)\right\rangle d t
$$

Da arbitrariedade de $\varphi$, concluímos que $\left\langle\left|u_{t}^{m}(t)\right|^{\rho} u_{t t}^{m}(t), \varphi(t)\right\rangle \rightarrow\left\langle\left|u_{t}(t)\right|^{\rho} u_{t t}(t), \varphi(t)\right\rangle \quad \forall \varphi \in H_{0}^{1}(\Omega)$ e para quase todo $t \in[0, \tau]$. 
- Limite para $f\left(u^{m}\right)$.

Procederemos de maneira análoga ao anterior caso. Pelo fato que $W^{1, \infty}\left(0, \tau ; H_{0}^{1}(\Omega)\right) \stackrel{c}{\hookrightarrow}$ $C\left([0, \tau] ; L^{2}(\Omega)\right)$ e passando a uma subsequencia se for necessário, teremos que

$$
u^{m} \rightarrow u \text { q.s. em } \Omega \times[0, \tau]
$$

agora, dado que $f$ é continua temos que

$$
f\left(u^{m}\right) \rightarrow f(u) \text { q.s. em } \Omega \times[0, \tau] .
$$

Pela observação (3.3) notamos que $f\left(u^{m}\right)$ é uniformemente limitada em $L^{\frac{\rho+2}{\rho+1}}(\Omega)$ respeito de $t \in[0, \tau]$, obtemos que

$$
\int_{0}^{\tau}\left\langle f\left(u^{m}\right), \varphi\right\rangle d t \rightarrow \int_{0}^{\tau}\langle f(u), \varphi\rangle d t, \quad \varphi \in D\left([0, \tau] ; H_{0}^{1}(\Omega)\right) .
$$

Dado que $\overline{D(\Omega)}{ }^{H_{0}^{1}(\Omega)}=H_{0}^{1}(\Omega)$, por um passagem ao limite por densidade e continuidade, e dado que $\varphi$ é arbitrário, temos que

$$
\left\langle f\left(u^{m}\right), \varphi\right\rangle \rightarrow\langle f(u), \varphi\rangle \quad \forall \varphi \in H_{0}^{1}(\Omega) \text { e para quase todo } t \in[0, \tau] .
$$

- Tratamento do Limite para a Equação (2.18).

Notemos que pela primeira estimativa temos que $\eta^{t, m} \in d o m(T)$, e procedendo igual que a observação (3.4), a representação para $\eta^{t, m}$ é dada por

$$
\eta^{t, m}(s)= \begin{cases}u^{m}(t)-u^{m}(t-s) & \text { se } \quad 0<s \leq t \\ \eta_{0}^{m}(s-t)+u^{m}(t)-u_{0}^{m} & \text { se } \quad s>t\end{cases}
$$

Ademais, como $u_{0}^{m} \rightarrow u_{0}$ e $\eta_{0}^{m} \rightarrow \eta_{0}$ fortemente, e tendo em conta novamente as convergências ao final da primeira estimativa, teremos que $\eta^{\bullet}, m \rightarrow \bar{\eta}^{\bullet}$ na topologia fraca em $L^{\infty}\left(\mathbb{R}^{+} ; \mathcal{M}\right)$ para certo $\bar{\eta} \in L^{\infty}\left(\mathbb{R}^{+} ; \mathcal{M}\right)$. Assim obtemos que

$$
\bar{\eta}^{t}(s)= \begin{cases}u(t)-u(t-s) & \text { se } \quad 0<s \leq t \\ \eta_{0}(s-t)+u(t)-u_{0} & \text { se } \quad s>t\end{cases}
$$

agora como $\eta^{\bullet, m} \stackrel{*}{\rightarrow} \eta^{\bullet}$ em $L^{\infty}\left(\mathbb{R}^{+} ; \mathcal{M}\right)$, obriga a que $\bar{\eta}=\eta$. Assim $\eta^{t}$ é uma solução suave para a equação (2.18), e em particular, dado que já temos a representação de $\eta^{t}$, esta cumpre a equação (3.2).

Assim, dadas as diferentes estimativas e tendo as respectivas aclarações respeito aos limites no problema aproximado, podemos garantir a existência de soluções fracas para o sistema (2.17)-(2.18), o que prova o Teorema 3.5. 


\subsection{Dependência contínua e unicidade das soluções fra- cas}

Nesta seção se seguira o trabalho feito por Conti, Marchini \& Pata [11], dado que eles apresentam a prova da dependência continua das soluções fracas com respeito aos dados iniciais e a unicidade das soluções fracas para nosso sistema (2.17)-(2.18).

Seja $\tau>0$ fixo mas arbitrário. Dado $\left(u^{1}, u_{t}^{1}, \eta^{\bullet, 1}\right),\left(u^{2}, u_{t}^{2}, \eta^{\bullet, 2}\right) \in C([0, \tau], \mathscr{H})$ duas soluções fracas para o sistema (2.17)-(2.18), satisfazendo os dados iniciais

$$
\left(u^{i}(0), u_{t}^{i}(0), \eta^{0, i}\right)=z_{i} \quad \forall i=1,2
$$

onde $z_{1}, z_{2} \in \mathscr{H}$.

Dado que queremos provar a unicidade das soluções, denotaremos a diferença de ditas soluções como

$$
\bar{u}=u^{1}-u^{2}, \quad \bar{\eta}=\eta^{\bullet, 1}-\eta^{\bullet, 2} .
$$

Notemos que $\bar{u}, \bar{\eta} \in C([0, \tau], \mathscr{H})$ e que pela linearidade da derivada temos que $\bar{u}_{t}=$ $u_{t}^{1}-u_{t}^{2}$.

\subsubsection{Dependência contínua das soluções fracas}

Agora enunciaremos e demostraremos o teorema que nos garante a dependência continua das soluções fracas com respeito aos dados iniciais, isto va ser de suma importância para a prova da unicidade.

Teorema 3.12: (Dependência Continua das Soluções Fracas Com Respeito aos Dados Iniciais).

Seja $R \geq 0$ e dadas as definições anteriores. Se $\left\|z_{i}\right\|_{\mathscr{H}} \leq R$ para $i=1,2$ então se cumpre que

$$
\|\nabla \bar{u}(t)\|_{2}^{2}+\left\|\nabla \bar{u}_{t}(t)\right\|_{2}^{2}+\left\|\bar{\eta}^{t}\right\|_{\mathcal{M}}^{2} \leq(1+\tau) Q(R) e^{\tau(\tau+1) Q(R)}\left\|z_{1}-z_{2}\right\|_{\mathscr{H}}^{2}
$$

para todo $t \in[0, \tau]$, onde $Q: \mathbb{R}^{+} \rightarrow \mathbb{R}^{+}$é uma função crescente independente de $\tau>0$.

Demonstração. Começaremos a prova introduzindo as seguintes variáveis

$$
w(t)=\int_{0}^{t} u(y) d y \quad \text { e } \quad \xi^{t}(s)=\int_{0}^{t} \eta^{y}(s) d y .
$$


Alem disso definimos a seguinte função $\sigma: H_{0}^{1}(\Omega) \rightarrow L^{\frac{\rho+2}{\rho+1}}(\Omega)$ tal que

$$
\sigma(\nu)=\frac{1}{1+\rho} \nu|\nu|^{\rho}
$$

\section{Observação 3.13:}

- Se $u \in H_{0}^{1}(\Omega)$, efetivamente, $\sigma(u) \in L^{\frac{\rho+2}{\rho+1}}(\Omega)$ pois

$$
\int_{\Omega}|\sigma(u)|^{\frac{\rho+2}{\rho+1}} d x=\int_{\Omega} \frac{1}{(1+\rho)^{\frac{\rho+2}{\rho+1}}}|u|^{\rho+2} d x=\frac{1}{(1+\rho)^{\frac{\rho+2}{\rho+1}}}\|u\|_{\rho+2}^{\rho+2} \leq \frac{C}{(1+\rho)^{\frac{\rho+2}{\rho+1}}}\|\nabla u\|_{2}^{\rho+2} \leq \infty
$$

onde $C>0$ é a constante produto da imersão $H_{0}^{1}(\Omega) \hookrightarrow L^{\rho+2}(\Omega)$.

- Igual que na observação $(3.3)$, como $L^{\frac{\rho+2}{\rho+1}}(\Omega)=\left(L^{\rho+2}(\Omega)\right)^{\prime}$ e dado que $H_{0}^{1}(\Omega) \hookrightarrow$ $\left.L^{\rho+2}(\Omega)\right)$, pois $\rho \in[0,4)$, temos que $L^{\frac{\rho+2}{\rho+1}}(\Omega) \hookrightarrow H^{-1}(\Omega)$. Assim vai ter sentido falar da expressão

$$
\langle\sigma(u), \varphi\rangle:=\langle\sigma(u), \varphi\rangle_{H^{-1}(\Omega) \times H_{0}^{1}(\Omega)}, \quad \forall \varphi \in H_{0}^{1}(\Omega) .
$$

Alem disso, vai estar dada por

$$
\langle\sigma(u), \varphi\rangle_{H^{-1}(\Omega) \times H_{0}^{1}(\Omega)}=\int_{\Omega} \sigma(u) \varphi d x \quad \forall \varphi \in H_{0}^{1}(\Omega),
$$

isto devido à caracterização das distribuição que pertencem a $L_{l o c}^{1}(\Omega)$.

- Dado que $\rho \in[0,4)$, a função $\sigma$ é monótona. (Vide Zeidler [40] pag. 502.).

- Dado $u, v \in \mathbb{R}$ teremos que

$$
|\sigma(u)-\sigma(v)| \leq c_{\sigma}\left(|u|^{\rho}+|v|^{\rho}\right)|u-v|
$$

onde $c_{\sigma}>0$ é uma constante que depende de $\rho$. Comprovaremos o resultado apenas para o caso $\rho \in(0,4)$, pois se $\rho=0$ a prova é trivial. Então notemos que

$$
|\sigma(u)-\sigma(v)|=\left.\frac{1}{1+\rho}|| u\right|^{\rho} u-\left.|v|^{\rho} v\left|=\frac{1}{\rho+1}(\rho+1)\right| \zeta\right|^{\rho}|u-v|,
$$


onde $\zeta \in[u, v]$, isto é pelo Teorema do Valor Meio. Agora seja $\theta \in[0,1]$ tal que $\zeta=(1-\theta) u+\theta v$, então teremos que

$$
\begin{aligned}
|\sigma(u)-\sigma(v)|=\left.\frac{1}{1+\rho}|| u\right|^{\rho} u-|v|^{\rho} v \mid & \leq|(1-\theta) u+\theta v|^{\rho}|u-v| \\
& \leq 2^{\rho}|| u-\left.\theta u\right|^{\rho}+|\theta v|^{\rho}|| u-v \mid \\
& \leq\left. 2^{\rho}\left|2^{\rho}\right| u\right|^{\rho}+\theta^{\rho}|u|^{\rho}+\theta^{\rho}|v|^{\rho}|| u-v \mid \\
& \leq\left. 2^{\rho}\left|2^{\rho}\right| u\right|^{\rho}+|u|^{\rho}+|v|^{\rho}|| u-v \mid \\
& \leq\left. 2^{\rho}\left|\left(2^{\rho}+1\right)\right| u\right|^{\rho}+|v|^{\rho}|| u-v \mid \\
& \leq\left. 2^{\rho}\left(2^{\rho}+1\right)|| u\right|^{\rho}+|v|^{\rho}|| u-v \mid \\
& =\left.c_{\sigma}|| u\right|^{\rho}+|v|^{\rho}|| u-v \mid .
\end{aligned}
$$

Com isto comprovamos o resultado.

Notemos que, integrando a equação (2.17) de 0 a $t$, obtemos

$$
\sigma\left(u_{t}\right)+A w_{t t}+A w+\int_{0}^{\infty} \mu(s) A \xi(s) d s+\int_{0}^{t} f(u(y)) d y=t h+g
$$

com

$$
g=A u_{t}(0)+\sigma\left(u_{t}(0)\right)
$$

Agora, construímos cada variável anterior para cada um das soluções $\left(u^{1}, u_{t}^{1}, \eta^{t, 1}\right)$ e $\left(u^{2}, u_{t}^{2}, \eta^{t, 2}\right)$. Assim teremos que

$$
w^{i}(t)=\int_{0}^{t} u^{i}(y) d y, \quad \xi^{t, i}=\int_{0}^{t} \eta^{y, i}(s) d y, \quad \forall i=1,2 .
$$

Alem disso, denotamos por

$$
\bar{w}(t)=w^{1}(t)-w^{2}(t) \quad \text { e } \quad \bar{\xi}^{t}=\xi^{t, 1}-\xi^{t, 2} .
$$

Notemos que $\left(\bar{w}, \bar{\xi}^{t}\right)$ satisfaz o sistema

$$
\begin{gathered}
\sigma\left(u_{t}^{1}\right)-\sigma\left(u_{t}^{2}\right)+A \bar{w}_{t t}+A \bar{w}+\int_{0}^{\infty} \mu(s) A \bar{\xi}^{t}(s) d s+F=G, \\
\bar{\xi}_{t}^{t}=T \bar{\xi}^{t}+\bar{w}_{t}-\bar{u}(0)+\bar{\eta}^{0} .
\end{gathered}
$$

com

$$
F(t)=\int_{0}^{t}\left[f\left(u^{1}(y)\right)-f\left(u^{2}(y)\right)\right] d y, \quad G=A \bar{u}_{t}(0)+\sigma\left(u_{t}^{1}(0)\right)-\sigma\left(u_{t}^{2}(0)\right) .
$$

Isto é fácil de comprovar, so basta restar a equação (3.23) e (2.18) nas respetivas variáveis referentes a cada solução fraca, e trocar pelas variáveis $\bar{w}$ e $\bar{\xi}^{t}$. 
Agora, aplicando $\bar{w}_{t t}=u_{t}^{1}-u_{t}^{2}$ na equação (3.24), e pela monotonia da função $\sigma$, obtemos

$$
\left\|\nabla \bar{w}_{t t}\right\|_{2}^{2} \leq-\left\langle\bar{w}, \bar{w}_{t t}\right\rangle_{1}-\int_{0}^{\infty} \mu(s)\left\langle\bar{\xi}^{t}(s), \bar{w}_{t t}\right\rangle_{1} d s-\left\langle F, \bar{w}_{t t}\right\rangle+\left\langle G, \bar{w}_{t t}\right\rangle
$$

Análogo à prova da existência de soluções, vamos a definir uma "Energia Auxiliar"para o problema.

Definição 3.14: Definimos o funcional

$$
\Lambda(t):=\frac{1}{2}\|\nabla \bar{w}(t)\|_{2}^{2}+\frac{1}{2}\left\|\nabla \bar{w}_{t}(t)\right\|_{2}^{2}+\frac{1}{2}\left\|\bar{\xi}^{t}\right\|_{\mathcal{M}}^{2}=\left\|\left(\bar{w}(t), \bar{w}_{t}(t), \bar{\xi}^{t}\right)\right\|_{\mathscr{H}}^{2} .
$$

Dito funcional sera chamado "Energia Auxiliar" para o sistema (3.24)-(3.25).

Neste ponto partiremos a prova do teorema em três etapas para poder estimar cada termo da expressão (3.20).

\section{Prova do Teorema: Estimação para $\|\nabla \bar{u}(t)\|_{2}^{2}$}

Notemos que

$$
\frac{d}{d t} \Lambda(t)=\left\langle\bar{w}(t), \bar{w}_{t}(t)\right\rangle_{1}+\left\langle\bar{w}_{t t}(t), \bar{w}_{t}(t)\right\rangle_{1}+\left\langle\bar{\xi}_{t}^{t}, \bar{\xi}^{t}\right\rangle_{\mathcal{M}}
$$

Então, aplicando $\bar{w}_{t t}$ na equação (3.24), aplicando $\bar{\xi}^{t}$ na equação (3.25) e pela igualdade (3.28), obtemos que

$$
\frac{d}{d t} \Lambda(t)=\left\langle T \bar{\xi}^{t}, \bar{\xi}^{t}\right\rangle_{\mathcal{M}}-\left\langle\sigma\left(u_{t}^{1}\right)-\sigma\left(u_{t}^{2}\right), \bar{w}_{t}\right\rangle-\left\langle F, \bar{w}_{t}\right\rangle+\left\langle G, \bar{w}_{t}\right\rangle+\left\langle\bar{\eta}^{0}-\bar{u}(0), \bar{\xi}^{t}\right\rangle_{\mathcal{M}}
$$

Ademais, notemos que

$$
\begin{gathered}
-\frac{d}{d t}\left\langle F, \bar{w}+\bar{w}_{t}\right\rangle+\left\langle F_{t}, \bar{w}+\bar{w}_{t}\right\rangle=-\left\langle F, \bar{w}_{t}\right\rangle-\left\langle F, \bar{w}_{t t}\right\rangle \\
\frac{d}{d t}\left\langle G, \bar{w}+\bar{w}_{t}\right\rangle=\left\langle G, \bar{w}_{t}+\bar{w}_{t t}\right\rangle
\end{gathered}
$$

agora, pelo resultado (2.13), e tendo em conta as igualdades (3.30)-(3.31), temos que a equação (3.29) se pode escrever como

$$
\begin{aligned}
\frac{d}{d t} \Lambda(t)+\left\|\nabla \bar{w}_{t t}\right\|_{2}^{2} \leq & -\left\langle\sigma\left(u_{t}^{1}\right)-\sigma\left(u_{t}^{2}\right), \bar{w}_{t}\right\rangle-\frac{d}{d t}\left\langle F, \bar{w}+\bar{w}_{t}\right\rangle+\left\langle F_{t}, \bar{w}+\bar{w}_{t}\right\rangle \\
& +\frac{d}{d t}\left\langle G, \bar{w}+\bar{w}_{t}\right\rangle+\left\langle\bar{\eta}^{0}-\bar{u}(0), \bar{\xi}^{t}\right\rangle_{\mathcal{M}}-\left\langle\bar{w}, \bar{w}_{t t}\right\rangle_{1}-\int_{0}^{\infty} \mu(s)\left\langle\bar{\xi}^{t}(s), \bar{w}_{t t}\right\rangle_{1} d s .
\end{aligned}
$$


Por outro lado, é fácil ver que pela hipóteses temos que existe uma função $Q_{0}: \mathbb{R}^{+} \rightarrow$ $\mathbb{R}^{+}$, crescente, independente do tempo, tal que

$$
\left\|\nabla u^{i}\right\|_{2}^{2}+\left\|\nabla u_{t}^{i}\right\|_{2}^{2}+\left\|\eta^{t, i}\right\|_{\mathcal{M}}^{2} \leq Q_{0}(R) \quad \forall i=1,2
$$

Observação 3.15: Para não sobre carregar de notações o trabalho, a partir de agora denotaremos por $Q(R)$ a qualquer função crescente, independente do tempo, que contenha a $Q_{0}(R)$.

Agora começaremos a estimar alguns termos da desigualdade acima

- Estimativa para $\left\langle\sigma\left(u_{t}^{1}\right)-\sigma\left(u_{t}^{2}\right), \bar{w}_{t}\right\rangle$

Pela observação (3.13), pelo fato de que $H_{0}^{1}(\Omega) \hookrightarrow L^{p+2}(\Omega)$ e como $\bar{w}_{t t}=u_{t}^{1}-u_{t}^{2}$, teremos que,

$$
\begin{aligned}
\left\langle\sigma\left(u_{t}^{1}\right)-\sigma\left(u_{t}^{2}\right), u_{t}^{1}-u_{t}^{2}\right\rangle \leq & c_{\sigma} \int_{\Omega}\left(\left|u_{t}^{1}\right|^{\rho}+\left|u_{t}^{2}\right|^{\rho}\right)\left|u_{t}^{1}-v_{t}^{1} \| \bar{w}_{t}\right| d x \\
= & c_{\sigma} \int_{\Omega}\left|u_{t}^{1}\right|^{\rho}\left|u_{t}^{1}-v_{t}^{1}\left\|\left.\bar{w}_{t}\left|d x+c_{\sigma} \int_{\Omega}\right| u_{t}^{2}\right|^{\rho}\left|u_{t}^{1}-v_{t}^{1} \| \bar{w}_{t}\right| d x\right.\right. \\
\leq & c_{\sigma}\left\|u_{t}^{1}\right\|_{\rho+2}^{\rho}\left\|u_{t}^{1}-u_{t}^{2}\right\|_{\rho+2}\left\|\bar{w}_{t}\right\|_{\rho+2} \\
& +c_{\sigma}\left\|u_{t}^{2}\right\|_{\rho+2}^{\rho}\left\|u_{t}^{1}-u_{t}^{2}\right\|_{\rho+2}\left\|\bar{w}_{t}\right\|_{\rho+2} \\
\leq & Q(R)\left\|\nabla u_{t}^{1}-\nabla u_{t}^{2}\right\|_{2}\left\|\nabla \bar{w}_{t}\right\|_{2}+Q(R)\left\|\nabla u_{t}^{1}-\nabla u_{t}^{2}\right\|_{2}\left\|\nabla \bar{w}_{t}\right\|_{2} \\
\leq & Q(R)\left\|\nabla u_{t}^{1}-\nabla u_{t}^{2}\right\|_{2}\left\|\nabla \bar{w}_{t}\right\|_{2} \\
\leq & \frac{1}{2}\left\|\nabla \bar{w}_{t t}\right\|_{2}^{2}+Q(R)\left\|\nabla \bar{w}_{t}\right\|_{2}^{2}
\end{aligned}
$$

Assim vemos que:

$$
\left\langle\sigma\left(u_{t}^{1}\right)-\sigma\left(u_{t}^{2}\right), u_{t}^{1}-u_{t}^{2}\right\rangle \leq \frac{1}{2}\left\|\nabla \bar{w}_{t t}\right\|_{2}^{2}+Q(R)\left\|\nabla \bar{w}_{t}\right\|_{2}^{2}
$$

Observação 3.16: Na estimativa acima, foi fortemente usado o fato de que

$$
\frac{\rho}{\rho+2}+\frac{1}{\rho+2}+\frac{1}{\rho+2}=1
$$

Esta igualdade vai ser usada ao largo de todo o trabalho.

- Estimativa para $\left\langle\bar{\eta}^{0}-\bar{u}(0), \bar{\xi}^{t}\right\rangle_{\mathcal{M}}$

$$
\left\langle\bar{\eta}^{0}-\bar{u}(0), \bar{\xi}^{t}\right\rangle_{\mathcal{M}} \leq\|\nabla \bar{u}(0)\|_{2}^{2}+\left\|\bar{\eta}^{0}\right\|_{\mathcal{M}}^{2}+c\left\|\bar{\xi}^{t}\right\|_{\mathcal{M}}^{2}
$$


Onde $c>0$ é uma constante produto da desigualdade de Young com épsilon.

- Estimativa para $\left\langle\bar{w}, \bar{w}_{t t}\right\rangle_{1}+\int_{0}^{\infty} \mu(s)\left\langle\bar{\xi}^{t}(s), \bar{w}_{t t}\right\rangle_{1} d s$

$$
\begin{aligned}
\left\langle\bar{w}, \bar{w}_{t t}\right\rangle_{1}+\int_{0}^{\infty} \mu(s)\left\langle\bar{\xi}^{t}(s), \bar{w}_{t t}\right\rangle_{1} d s & \leq\left(\|\nabla \bar{w}\|_{2}+k_{0}^{\frac{1}{2}}\left\|\bar{\xi}^{t}\right\|_{\mathcal{M}}\right)\left\|\nabla \bar{w}_{t t}\right\|_{2} \\
& \leq c\left(\|\nabla \bar{w}\|_{2}^{2}+\left\|\bar{\xi}^{t}\right\|_{\mathcal{M}}^{2}\right)+\frac{1}{2}\left\|\nabla \bar{w}_{t t}\right\|_{2}^{2}
\end{aligned}
$$

Então,

$$
\left\langle\bar{w}, \bar{w}_{t t}\right\rangle_{1}+\int_{0}^{\infty} \mu(s)\left\langle\bar{\xi}^{t}(s), \bar{w}_{t t}\right\rangle_{1} d s \leq c\left(\|\nabla \bar{w}\|_{2}^{2}+\left\|\bar{\xi}^{t}\right\|_{\mathcal{M}}^{2}\right)+\frac{1}{2}\left\|\nabla \bar{w}_{t t}\right\|_{2}^{2}
$$

Onde $c>0$ é uma constante produto da desigualdade de Young que também depende de $k_{0}$.

Estas dois ultimas estimativas se fazem análogo ao processo visto na seção anterior, na "Segunda Estimativa".

Dadas estas estimativas, nossa desigualdade se pode escrever como

$$
\frac{d}{d t} \Lambda(t) \leq Q(R) \Lambda(t)-\frac{d}{d t}\left\langle F, \bar{w}+\bar{w}_{t}\right\rangle+\frac{d}{d t}\left\langle G, \bar{w}+\bar{w}_{t}\right\rangle+\left\langle F_{t}, \bar{w}+\bar{w}_{t}\right\rangle+\|\nabla \bar{u}(0)\|_{2}^{2}+\left\|\bar{\eta}^{0}\right\|_{\mathcal{M}}^{2}
$$

Agora, tomemos $s \in[0, \tau]$ tal que integramos (3.35) no intervalo $(0, s)$ respeito de $t$, então teremos que

$$
\begin{aligned}
\Lambda(s) \leq & Q(R) \int_{0}^{s} \Lambda(t) d t-\left\langle F(s), \bar{w}(s)+\bar{w}_{t}(s)\right\rangle+\left\langle G, \bar{w}(s)+\bar{w}_{t}(s)\right\rangle \\
& -\langle G, \bar{u}(0)\rangle+\int_{0}^{s}\left\langle F_{t}(t), \bar{w}(t)+\bar{w}_{t}(t)\right\rangle d t+(1+s)\|\nabla \bar{u}(0)\|_{2}^{2}+s\left\|\bar{\eta}^{0}\right\|_{\mathcal{M}}^{2}
\end{aligned}
$$

Mas, notemos que

$$
\begin{aligned}
\left\langle F(s), \bar{w}(s)+\bar{w}_{t}(s)\right\rangle & \leq\|F(s)\|_{H^{-1}(\Omega)}\left\|\nabla \bar{w}(s)+\nabla \bar{w}_{t}(s)\right\|_{2} \\
& \leq \epsilon\|F(s)\|_{H^{-1}(\Omega)}^{2}+\frac{1}{2 \epsilon}\left(\|\nabla \bar{w}(s)\|_{2}^{2}+\left\|\nabla \bar{w}_{t}(s)\right\|_{2}^{2}\right) \\
& \leq \epsilon\|F(s)\|_{H^{-1}(\Omega)}^{2}+\frac{1}{\epsilon} \Lambda(s)
\end{aligned}
$$


$\operatorname{com} \epsilon>0$ da desigualdade de Young com épsilon. Seja $\delta>0$ então, procedendo de maneira análoga teremos que

$$
\left\langle G, \bar{w}(s)+\bar{w}_{t}(s)\right\rangle \leq \delta\|G(s)\|_{H^{-1}(\Omega)}^{2}+\frac{1}{\delta} \Lambda(s) .
$$

Assim, ajustando os valores para $\epsilon$ e $\delta$ temos que

$$
\begin{aligned}
\left\langle F(s), \bar{w}(s)+\bar{w}_{t}(s)\right\rangle+\left\langle G, \bar{w}(s)+\bar{w}_{t}(s)\right\rangle-\langle G, \bar{u}(0)\rangle \leq & c\|F(s)\|_{H^{-1}(\Omega)}^{2}+c\|G\|_{H^{-1}(\Omega)}^{2} \\
& +\frac{1}{2} \Lambda(s)+c\|\nabla \bar{u}(0)\|_{2}^{2},
\end{aligned}
$$

onde $c>0$ é uma constante que depende dos valores escolhidos para $\epsilon$ e $\delta$ de tal maneira que o coeficiente de $\Lambda(s)$ seja $\frac{1}{2}$.

Substituindo (3.37) em (3.2.1), obtemos que

$$
\begin{aligned}
\Lambda(s) \leq & Q(R) \int_{0}^{s} \Lambda(t) d t+c\|F(s)\|_{H^{-1}(\Omega)}^{2}+c\|G\|_{H^{-1}(\Omega)}^{2} \\
& +\int_{0}^{s}\left\langle F_{t}(t), \bar{w}(t)+\bar{w}_{t}(t)\right\rangle d t+c(1+s)\left\|z_{1}-z_{2}\right\|_{\mathscr{H}}^{2} .
\end{aligned}
$$

Novamente, vamos a estimar termo por termo do lado direito da desigualdade acima.

- Estimativa para $\|F(s)\|_{H^{-1}(\Omega)}^{2}$

Notemos que como $q \in[0,4)$, pela hipóteses $(2.2)$ e pela imersão $L^{\frac{\rho+2}{\rho+1}}(\Omega) \hookrightarrow H^{-1}(\Omega)$ temos que

$$
\begin{aligned}
\|F(s)\|_{H^{-1}(\Omega)}^{\frac{q+2}{q+1}} & \leq C\|F(s)\|_{\frac{q+2}{q+1}}^{\frac{q+2}{q+1}} \\
& \leq C\left[\int_{\Omega}\left(\int_{0}^{s} c\left|u^{1}(y)-u^{2}(y)\right|\left(1+\left|u^{1}(y)\right|^{q}+\left|u^{2}(y)\right|^{q}\right) d y\right]^{\frac{q+2}{q+1}} d x\right. \\
& \leq C s^{\frac{1}{q+1}} c^{\frac{q+2}{q+1}} \int_{0}^{s} \int_{\Omega}\left(1+\left|u^{1}(y)\right|^{q}+\left|u^{2}(y)\right|^{q}\right)^{\frac{q+2}{q+1}}\left|u^{1}(y)-u^{2}(y)\right|^{\frac{q+2}{q+1}} d x d y \\
& \leq C s^{\frac{1}{q+1}} c^{\frac{q+2}{q+1}} \int_{0}^{s}\left(\int_{\Omega}^{s}\left(1+\left|u^{1}(y)\right|^{q}+\left|u^{2}(y)\right|^{q}\right)^{\frac{q+2}{q+1} \frac{q+1}{q}} d x\right)^{\frac{q}{q+1}}\left(\int_{\Omega}|\bar{u}(y)|^{q+2} d x\right)^{\frac{1}{q+1}} d y \\
& \leq s^{\frac{1}{q+1}} Q(R) \int_{0}^{s}\|\nabla \bar{u}(y)\|_{2}^{\frac{q+2}{q+1}} d y,
\end{aligned}
$$

onde, $C>0$ é a constante da imersão $L^{\frac{q+2}{q+1}}(\Omega) \hookrightarrow H^{-1}(\Omega)$ e $c>0$ vem da hipóteses (2.2). Notemos também que a ultima desigualdade se debe a que $H_{0}^{1}(\Omega) \hookrightarrow L^{\frac{q+2}{q+1}}(\Omega)$. Alem disso, $Q(R)$ depende de $c, C, q,|\Omega|, R$. 
Agora, como $\frac{2}{q+2}<1$

$$
\begin{aligned}
\|F(s)\|_{H^{-1}(\Omega)}^{2} & \leq s^{\frac{2}{q+2}} Q(R)\left(\int_{0}^{s}\|\nabla \bar{u}(y)\|_{2}^{\frac{q+2}{q+1}} d y\right)^{\frac{2(q+1)}{q+2}} \\
& \leq s Q(R) \int_{0}^{s}\|\nabla \bar{u}(y)\|_{2}^{2} d y \\
& \leq s Q(R) \int_{0}^{s} \Lambda(y) d y .
\end{aligned}
$$

- Estimativa para $\|G\|_{H^{-1}(\Omega)}^{2}$

Repetindo as contas da estimativa (3.32), teremos que

$$
\begin{aligned}
\left\|\sigma\left(u_{t}^{1}(0)\right)-\sigma\left(u_{t}^{2}(0)\right)\right\|_{\frac{\rho+2}{\rho+1}}^{\frac{\rho+2}{\rho+1}} & \leq c_{\sigma} \int_{\Omega}\left(\left|u_{t}^{1}(0)\right|^{\rho}+\left|u_{t}^{2}(0)\right|^{\rho}\right)^{\frac{\rho+2}{\rho+1}}\left|\bar{u}_{t}(0)\right|^{\frac{\rho+2}{\rho+1}} d x \\
& \leq Q(R)\left\|\nabla \bar{u}_{t}(0)\right\|_{2}^{\frac{\rho+2}{\rho+1}} .
\end{aligned}
$$

Assim obtemos que

$$
\|G\|_{H^{-1}(\Omega)}^{2} \leq Q(R)\left\|\nabla \bar{u}_{t}(0)\right\|_{2}^{2}
$$

- Estimativa para $\int_{0}^{s}\left\langle F_{t}(t), \bar{w}(t)+\bar{w}_{t}(t)\right\rangle d t$

Razonando igual que as anteriores estimativas, obtemos que

$$
\left\|F_{t}\right\|_{\frac{\rho+2}{\rho+1}}^{\frac{\rho+2}{\rho+1}} \leq c \int_{\Omega}\left(1+\left|u^{1}\right|^{\rho}+\left|u^{2}\right|^{\rho}\right)^{\frac{\rho+2}{\rho+1}}|\bar{u}|^{\frac{\rho+2}{\rho+1}} d x \leq Q(R)\|\nabla \bar{u}\|_{2}^{\frac{\rho+2}{\rho+1}}
$$

onde $c>0$ é a constante da hipóteses (2.2).

Então teremos que

$$
\begin{aligned}
\int_{0}^{s}\left\langle F_{t}(t), \bar{w}(t)+\bar{w}_{t}(t)\right\rangle d t & \leq \int_{0}^{s}\left\|F_{t}(t)\right\|_{\frac{\rho+2}{\rho+1}}\left(\|\bar{w}(t)\|_{\rho+2}+\left\|\bar{w}_{t}(t)\right\|_{\rho+2}\right) d t \\
& \leq Q(R) \int_{0}^{s} \Lambda(t) d t
\end{aligned}
$$

Assim, substituindo as estimativas acima na desigualdade (3.38), teremos que

$$
\Lambda(s) \leq(1+s) Q(R) \int_{0}^{s} \Lambda(t) d t+(1+s) Q(R)\left\|z_{1}-z_{2}\right\|_{\mathscr{H}}^{2}, \quad \forall s \in[0, \tau]
$$


Finalmente aplicando a desigualdade de Gronwall, obtemos que

$$
\Lambda(s) \leq(1+\tau) Q(R) e^{\tau(1+\tau) Q(R)}\left\|z_{1}-z_{2}\right\|_{\mathscr{H}}^{2}, \quad \forall s \in[0, \tau] .
$$

Em particular,

$$
\|\nabla \bar{u}(s)\|_{2}^{2} \leq(1+\tau) Q(R) e^{\tau(1+\tau) Q(R)}\left\|z_{1}-z_{2}\right\|_{\mathscr{H}}^{2}, \quad \forall s \in[0, \tau]
$$

\section{Prova do Teorema: Estimação para $\left\|\nabla \bar{u}_{t}\right\|_{2}^{2}$}

A estimação para $\bar{u}_{t}$ vem diretamente de igualdade (3.26), pois $\bar{w}_{t t}=u_{t}^{1}-u_{t}^{2}=\bar{u}_{t}$. Assim, tendo em conta dita igualdade e a estimativa (3.34), teremos que

$$
\left\|\nabla \bar{u}_{t}\right\|_{2}^{2} \leq \frac{1}{2}\left(\|\nabla \bar{w}\|_{2}+k_{0}^{\frac{1}{2}}\left\|\bar{\xi}^{t}\right\|_{\mathcal{M}}+\|F\|_{H^{-1}(\Omega)}+\|G\|_{H^{-1}(\Omega)}\right)^{2}+\frac{1}{2}\left\|\nabla \bar{u}_{t}\right\|_{2}^{2}
$$

e pela desigualdade (3.39) e as estimativas sobre $\|F\|_{H^{-1}(\Omega)}$ e $\|G\|_{H^{-1}(\Omega)}$, obtemos

$$
\left\|\nabla \bar{u}_{t}(s)\right\|_{2}^{2} \leq(1+\tau) Q(R) e^{\tau(1+\tau) Q(R)}\left\|z_{1}-z_{2}\right\|_{\mathscr{H}}^{2}, \quad \forall s \in[0, \tau] .
$$

\section{Prova do Teorema: Estimação para $\left\|\bar{\eta}^{t}\right\|_{\mathcal{M}}^{2}$}

Finalmente, para estimar $\left\|\bar{\eta}^{t}\right\|_{\mathcal{M}}^{2}$, primeiro apliquemos $\bar{\eta}$ na equação

$$
\bar{\eta}_{t}=T \bar{\eta}+\bar{u}_{t}
$$

Procedendo de maneira análoga que na seção anterior, teremos que

$$
\frac{d}{d t}\|\bar{\eta}\|_{\mathcal{M}}^{2}=\langle T \bar{\eta}, \bar{\eta}\rangle_{\mathcal{M}}+\left\langle\bar{u}_{t}, \bar{\eta}\right\rangle_{\mathcal{M}} \leq k_{0}^{\frac{1}{2}}\left\|\nabla \bar{u}_{t}\right\|_{2}\|\bar{\eta}\|_{\mathcal{M}} \leq\|\bar{\eta}\|_{\mathcal{M}}^{2}+c\left\|\nabla \bar{u}_{t}\right\|_{2}^{2}
$$

onde $c>0$ é uma constate que se obtêm da desigualdade de Young com épsilon.

Aplicando a desigualdade de Gronwall em (3.40), e tendo em conta a estimativa sobre $\left\|\nabla \bar{u}_{t}\right\|_{2}$, obtemos que

$$
\left\|\bar{\eta}^{s}\right\|_{\mathcal{M}}^{2} \leq(1+\tau) Q(R) e^{\tau(1+\tau) Q(R)}\left\|z_{1}-z_{2}\right\|_{\mathscr{H}}^{2}, \quad \forall s \in[0, \tau] .
$$

Assim, somando todas as estimativas teremos que

$\|\nabla \bar{u}(s)\|_{2}^{2}+\left\|\nabla \bar{u}_{t}(s)\right\|_{2}^{2}+\left\|\bar{\eta}^{s}\right\|_{\mathcal{M}}^{2} \leq(1+\tau) Q(R) e^{\tau(\tau+1) Q(R)}\left\|z_{1}-z_{2}\right\|_{\mathscr{H}}^{2}, \quad \forall s \in[0, \tau]$,

o que prova o teorema. 


\subsubsection{Unicidade das soluções fracas}

Teorema 3.17: (Unicidade das Soluções Fracas). Seja $\tau>0$ fixo mas arbitrário. Dado o sistema (2.17)-(2.18), com condições de fronteira (2.19) e dados iniciais (2.20). Suponha certa as hipóteses (H1) - (H4) . Se os dados iniciais $\left(u_{0}, v_{0}, \eta_{0}\right) \in \mathscr{H}$ e $h \in$ $L^{2}(\Omega)$, então o sistema (2.17)-(2.18) possui uma única solução fraca para todo $t \in[0, \tau]$.

Demonstração. Pelo Teorema 3.5, se tem que existem soluções fracas para o sistema com dados iniciais $(2.20)$.

Sejam $\left(u, u_{t}, \eta^{t}\right)$ e $\left(v, v_{t}, \xi^{t}\right)$ dois soluções para o sistema (2.17)-(2.18) com dados iniciais $(2.20)$.

Assim, pelo Teorema 3.12 temos que

$$
\|\nabla \bar{u}(t)\|_{2}^{2}+\left\|\nabla \bar{u}_{t}(t)\right\|_{2}^{2}+\left\|\bar{\eta}^{t}\right\|_{\mathcal{M}}^{2} \leq(1+\tau) Q(R) e^{\tau(\tau+1) Q(R)}\left\|z_{1}-z_{2}\right\|_{\mathscr{H}}^{2} \quad \forall t \in[0, \tau] .
$$

onde

$$
\bar{u}=u-v, \quad \bar{\eta}^{t}=\eta^{t}-\xi^{t}, \quad z_{1}=\left(u_{0}, v_{0}, \eta_{0}\right)=z_{2}
$$

Então, é claro que $\left(u, u_{t}, \eta^{t}\right)=\left(v, v_{t}, \xi^{t}\right)$ para todo $t \in[0, \tau]$, o que prova a unicidade. 


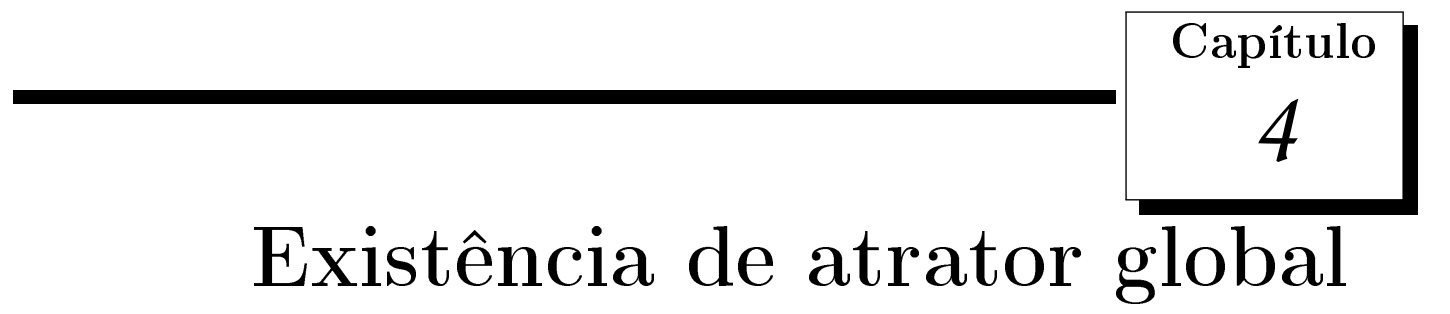

Neste Capitulo apresentaremos uma das nossas principais contribuições, provaremos a existência de um atrator global para o problema (2.17)-(2.18). Começaremos o capitulo definindo o $C_{0}$-semigrupo associado a dito sistema,

Teorema 4.1: Dado o sistema (2.17)-(2.18) seu operador solução dado por $S(t): \mathscr{H} \rightarrow$ $\mathscr{H}$ tal que

$$
S(t)\left(u_{0}, v_{0}, \eta_{0}\right)=\left(u(t), u_{t}(t), \eta^{t}\right), \quad t \geq 0
$$

onde $\left(u(t), u_{t}(t), \eta^{t}\right)$ é uma solução fraca correspondente ao sistema (2.17)-(2.18), define um $C_{0}$-semigrupo.

Demonstração. Notemos que pelo capitulo anterior, dito operador solução $S(t): \mathscr{H} \rightarrow \mathscr{H}$ satisfaz as propriedades de semigrupo, ou seja

$$
S(0)=I \quad \text { e } \quad S(t+s)=S(t) S(s), \quad s, t \geq 0
$$

isto é devido à unicidade das soluções fracas sempre que se fixa o dado inicial.

Agora provaremos que $S(t)$ é fortemente continuo.

Seja $z=\left(u_{0}, v_{0}, \eta_{0}\right) \in \mathscr{H}$ então dado que $S(t) z=\left(u, u_{t}, \eta^{t}\right)$ tal que $\left(u(0), u_{t}(0), \eta^{0}\right)=$ $z=\left(u_{0}, v_{0}, \eta_{0}\right)$ e como $\left(u, u_{t}, \eta^{t}\right) \in C([0, \tau], \mathscr{H})$ para todo $\tau>0$ (vide (3.4)), e pela dependência continua respeito aos dados inicias, teremos que

$$
\lim _{t \rightarrow 0^{+}}\|S(t) z-z\|_{\mathscr{H}}=0
$$

o que prova o teorema. 
Notemos que pelo teorema anterior teremos que o par $(\mathscr{H}, S(t))$ é um sistema dinâmico correspondente ao sistema (2.17)-(2.18).

Teorema 4.2: (Existência de Atrator Global).

Dado o sistema (2.17)-(2.18). Suponha certa as hipóteses (H1) - (H4) com $h \in$ $L^{2}(\Omega)$, então o sistema dinâmico $(\mathscr{H}, S(t))$ correspondente a dito sistema possui atrator global.

Demonstração. O método usado para a prova do atrator é dado pelo Teorema 1.28, o que reduz-se a provar que o sistema dinâmico $(\mathscr{H}, S(t))$ seja dissipativo e assintoticamente compacto.

Para provar isto, dividiremos a prova em três etapas. Primeiro provaremos a existência de um conjunto absorvente limitado para o sistema dinâmico $(\mathscr{H}, S(t))$, isto garante que dito sistema é dissipativo.

Na segunda etapa se mostrara um dos resultados mais importantes de todo o trabalho, se provara que o sistema dinâmico $(\mathscr{H}, S(t))$ cumpre uma desigualdade de estabilização, isto ajudara a provar que $(\mathscr{H}, S(t))$ é assintoticamente regular.

Finalmente na terceira etapa provaremos a compacidade assintótica, para o qual dividiremos a prova em dois resultados, o primeiro consistirá em provar que o sistema é assintoticamente regular, isto vai ser possível pela desigualdade de estabilização que se prova na segunda etapa e os Teoremas 1.31 e 1.17. No segundo resultado provaremos diretamente a compacidade assintótica pelo Teorema 1.30 .

Observação 4.3: Para facilitar as contas se terá as seguintes considerações.

- Omitiremos a variável temporal $t$. Só se colocará em casos onde se tenha alguma confusão.

- Denota-se por $C$ a qualquer constante genérica que dependa de alguma imersão.

- Dado que $\rho \in[0,4), q \in[0,4)$ e $2^{*}=6$ se tem as seguintes imersões

$$
H_{0}^{1}(\Omega) \hookrightarrow L^{\rho+2}(\Omega), \quad H_{0}^{1}(\Omega) \hookrightarrow L^{q+2}(\Omega)
$$


- Dado que $\frac{p}{p+2}+\frac{1}{p+2}+\frac{1}{p+2}=1$ para $p=\rho$ o $p=q$, se usara muito a desigualdade de Holder generalizada com ditos expoentes diretamente. Quando seja necessário se colocará em evidencia a soma anterior.

- Procedendo igual que no Capitulo 3, na prova da Primeira Estimativa (vide (3.17), (3.14) e (3.16)), teremos que se cumpre

$$
\begin{aligned}
2 \mathcal{E}(t)=\|\nabla u(t)\|_{2}^{2}+\left\|\nabla u_{t}(t)\right\|_{2}^{2}+\left\|\eta^{t}\right\|_{\mathcal{M}}^{2} & \leq \frac{2 E(t)+2 C_{h}}{\nu} \\
& \leq \frac{2 E(0)+2 C_{h}}{\nu} \\
& \leq C_{0 \nu}+C_{h \nu}
\end{aligned}
$$

onde $C_{h}=\frac{1}{\lambda_{1}}\|h\|_{2}^{2}, C_{h \nu}$ é um valor genérico positivo que depende de $\|h\|_{2}, \lambda_{1}$ e $\nu$, e $C_{0 \nu}$ um valor genérico positivo que depende dos dados inicias e de $\nu$, com $\nu>0$ um valor fixo que se obtêm da hipóteses (2.3).

\subsection{Dissipatividade}

Proposição 4.4: $\quad$ O sistema dinâmico $(\mathscr{H}, S(t))$ correspondente ao sistema (2.17)-(2.18) possui um conjunto absorvente limitado.

Demonstração. Seja o funcional $J:[0, \infty) \rightarrow \mathbb{R}$ tal que

$$
J(t):=E(t)+\epsilon \psi(t), \quad \epsilon>0,
$$

onde $E(t)$ é energia do sistema definido em (3.12) e $\psi$ é dado por

$$
\psi(t)=\frac{1}{\rho+1} \int_{\Omega}\left|u_{t}(t)\right|^{\rho} u_{t}(t) u(t) d x+\left\langle u_{t}(t), u(t)\right\rangle_{1} .
$$

Observação 4.5: Análogo a como se provo na observação (3.3), a integral $\int_{\Omega}\left|u_{t}(t)\right|^{\rho} u_{t}(t) u(t) d x$ no operador $\psi(t)$ é entendida como dualidade entre $H^{-1}(\Omega)$ e $H_{0}^{1}(\Omega)$. Esta observação foi primeiramente colocada no trabalho de Cavalcanti, Domingos Cavalcanti \& Ferreira [6].

Lema 4.6: Existe um $\epsilon_{0}>0$, que depende dos dados iniciais (2.20) tal que

$$
\frac{1}{2} E(t)-\frac{C_{h}}{2} \leq J(t) \leq \frac{3}{2} E(t)+\frac{C_{h}}{2}, \quad t \geq 0, \epsilon \in\left(0, \epsilon_{0}\right]
$$

onde $C_{h}=\frac{1}{\lambda_{1}}\|h\|_{2}^{2}>0$.

Demonstração. 
- Analisemos $|\psi(t)|$.

Notemos que

$$
\begin{aligned}
\left.\left|\int_{\Omega}\right| u_{t}(t)\right|^{\rho} u_{t}(t) u(t) d x \mid & \leq \int_{\Omega}\left|u_{t}\right|^{\rho}\left|u_{t} \| u\right| d x \\
& \leq\left(\int_{\Omega}\left|u_{t}\right|^{\rho+2} d x\right)^{\frac{\rho}{\rho+2}}\left(\int_{\Omega}\left|u_{t}\right|^{\rho+2} d x\right)^{\frac{1}{\rho+2}}\left(\int_{\Omega}|u|^{\rho+2} d x\right)^{\frac{1}{\rho+2}} \\
& \leq C\left\|\nabla u_{t}\right\|_{2}^{\rho}\left\|\nabla u_{t}\right\|_{2}\|\nabla u\|_{2} \\
& \leq C\left(C_{0 \nu}+C_{h \nu}\right)^{\rho / 2}\left\|\nabla u_{t}\right\|_{2}\|\nabla u\|_{2} \\
& \leq \frac{\left(C_{0 \nu}+C_{h \nu}\right)^{\rho}}{2}\left\|\nabla u_{t}\right\|_{2}^{2}+\frac{C^{2}}{2}\|\nabla u\|_{2}^{2} .
\end{aligned}
$$

Assim,

$$
\begin{aligned}
|\psi(t)| & \leq \frac{\left(C_{0 \nu}+C_{h \nu}\right)^{\rho}}{2}\left\|\nabla u_{t}\right\|_{2}^{2}+\frac{C^{2}}{2}\|\nabla u\|_{2}^{2}+\left|\left\langle u_{t}, u\right\rangle_{1}\right| \\
& \leq \frac{\left(C_{0 \nu}+C_{h \nu}\right)^{\rho}}{2}\left\|\nabla u_{t}\right\|_{2}^{2}+\frac{C^{2}}{2}\|\nabla u\|_{2}^{2}+\frac{1}{2}\left\|\nabla u_{t}\right\|_{2}^{2}+\frac{1}{2}\|\nabla u\|_{2}^{2} \\
& =\left(\frac{\left(C_{0 \nu}+C_{h \nu}\right)^{\rho}+1}{2}\right)\left\|\nabla u_{t}\right\|_{2}^{2}+\left(\frac{C^{2}+1}{2}\right)\|\nabla u\|_{2}^{2} \\
& \leq \frac{C_{1}}{2}\left(\left\|\nabla u_{t}\right\|_{2}^{2}+\|\nabla u\|_{2}^{2}\right) \\
& \leq C_{1}(\mathcal{E}(t)) \\
& \leq C_{1}\left(\frac{E(t)+C_{h}}{\nu}\right)
\end{aligned}
$$

onde $C_{1}=\max \left\{\left(C_{0 \nu}+C_{h \nu}\right)^{\rho}, C^{2}+1\right\}$. Tomando $\epsilon_{0}=\min \left\{\frac{\nu}{2 C_{1}}, 1\right\}$ então teremos que

$$
|\epsilon \psi(t)| \leq \frac{1}{2} E(t)+\frac{C_{h}}{2}, \quad \forall \epsilon \in\left(0, \epsilon_{0}\right]
$$

Assim temos que

$$
-\frac{1}{2} E(t)-\frac{C_{h}}{2} \leq \epsilon \psi(t) \leq \frac{1}{2} E(t)+\frac{C_{h}}{2},
$$

isto é,

$$
\frac{1}{2} E(t)-\frac{C_{h}}{2} \leq J(t) \leq \frac{3}{2} E(t)+\frac{C_{h}}{2}, \quad t \geq 0, \epsilon \in\left(0, \epsilon_{0}\right]
$$

Lema 4.7: Existe uma constante $C_{0}>0$ que depende dos dados inicias, $m_{f}$ e $|\Omega|$ tal que

$$
\psi^{\prime}(t) \leq-E(t)-C_{0} \int_{0}^{\infty} \mu^{\prime}(s)\left\|\nabla \eta^{t}(s)\right\|_{2}^{2} d s+C_{0}, \quad t \geq 0
$$


Demonstração. Como

$$
\psi(t)=\frac{1}{\rho+1} \int_{\Omega}\left|u_{t}\right|^{\rho} u_{t} u d x+\left\langle u_{t}, u\right\rangle_{1}
$$

então teremos que

$$
\psi^{\prime}(t)=\int_{\Omega}\left(\left|u_{t}\right|^{\rho} u_{t t}+A u_{t t}\right) u d x+\frac{1}{\rho+1}\left\|u_{t}\right\|_{\rho+2}^{\rho+2}+\left\|\nabla u_{t}\right\|_{2}^{2} .
$$

Substituindo na equação (2.17) teremos que

$$
\int_{\Omega}\left(\left|u_{t}\right|^{\rho} u_{t t}+A u_{t t}\right) u d x=-\|\nabla u\|_{2}^{2}-\int_{0}^{\infty} \mu(s)\left\langle\eta^{t}(s), u\right\rangle_{1} d s+(h, u)-\int_{\Omega} f(u) u d x
$$

Procedendo de maneira análoga a (3.18) e aplicando a desigualdade de Young com $\epsilon=2$ teremos que

$$
\int_{0}^{\infty} \mu(s)\left\langle\eta^{t}(s), u\right\rangle_{1} d s \leq \frac{1}{8}\|\nabla u\|_{2}^{2}+2 k_{0}\|\eta\|_{\mathcal{M}}^{2}
$$

Assim obtemos

$$
\begin{aligned}
-\|\nabla u\|_{2}^{2}-\int_{0}^{\infty} \mu(s)\left\langle\eta^{t}(s), u\right\rangle_{1} d s+(h, u)-\int_{\Omega} f(u) u d x \leq & \frac{1}{8}\|\nabla u\|_{2}^{2}+2 k_{0}\|\eta\|_{\mathcal{M}}^{2}-\|\nabla u\|_{2}^{2} \\
& +(h, u)-\int_{\Omega} f(u) u d x,
\end{aligned}
$$

e comparando com $\psi^{\prime}(t)$ temos

$$
\psi^{\prime}(t) \leq-\frac{7}{8}\|\nabla u\|_{2}^{2}+2 k_{0}\|\eta\|_{\mathcal{M}}^{2}+(h, u)-\int_{\Omega} f(u) u d x+\frac{1}{\rho+1}\left\|u_{t}\right\|_{\rho+2}^{\rho+2}+\left\|\nabla u_{t}\right\|_{2}^{2} .
$$

Agora, somando e restando $E(t)$ obtemos

$$
\psi^{\prime}(t) \leq-E(t)-\frac{3}{8}\|\nabla u\|_{2}^{2}+\left(\frac{1}{2}+2 k_{0}\right)\|\eta\|_{\mathcal{M}}^{2}+\langle\widehat{f}(u), 1\rangle-\int_{\Omega} f(u) u d x+\frac{2}{\rho+1}\left\|u_{t}\right\|_{\rho+2}^{\rho+2}+\frac{3}{2}\left\|\nabla u_{t}\right\|_{2}^{2}
$$

Por outro lado, pela hipóteses (2.4) e pela observação (1.13) temos que

$$
\begin{aligned}
\langle\widehat{f}(u), 1\rangle-\int_{\Omega} f(u) u d x & =\int_{\Omega}(\widehat{f}(u)-f(u) u) d x \\
& \leq \frac{\beta}{2} \int_{\Omega} u^{2} d x+m_{f}|\Omega| \\
& =\frac{\beta}{2 \lambda_{1}}\|\nabla u\|_{2}^{2}+m_{f}|\Omega|,
\end{aligned}
$$


alem disso, como $\beta \in\left(0, \lambda_{1}\right)$ então

$$
\langle\widehat{f}(u), 1\rangle-\int_{\Omega} f(u) u d x \leq \frac{1}{2}\|\nabla u\|_{2}^{2}+m_{f}|\Omega| .
$$

Substituindo isto em (4.2), e como $-\frac{7}{8}+\frac{1}{2}<0$ obtemos

$$
\psi^{\prime}(t) \leq-E(t)+\left(\frac{1}{2}+2 k_{0}\right)\|\eta\|_{\mathcal{M}}^{2}+\frac{2}{\rho+1}\left\|u_{t}\right\|_{\rho+2}^{\rho+2}+\frac{3}{2}\left\|\nabla u_{t}\right\|_{2}^{2}+m_{f}|\Omega| .
$$

Agora, pela hipóteses $(2.7)$ e pela imersão $H_{0}^{1}(\Omega) \hookrightarrow L^{\rho+2}(\Omega)$ teremos

$$
\psi^{\prime}(t) \leq-E(t)-\frac{4 k_{0}+1}{k_{1}} \int_{0}^{\infty} \mu^{\prime}(s)\left\|\nabla \eta^{t}(s)\right\|_{2}^{2} d s+C\left\|\nabla u_{t}\right\|_{2}^{2}+m_{f}|\Omega| .
$$

O que é equivalente a

$$
\psi^{\prime}(t) \leq-E(t)-\frac{4 k_{0}+1}{k_{1}} \int_{0}^{\infty} \mu^{\prime}(s)\left\|\nabla \eta^{t}(s)\right\|_{2}^{2} d s+C\left(C_{0 \nu}+C_{h \nu}\right)+m_{f}|\Omega|,
$$

Finalmente, como $\mu^{\prime}(s) \leq 0$ pela hipóteses $(2.5)$ e tomando $C_{0}=\max \left\{\frac{4 k_{0}+1}{k_{1}}, C\left(C_{0 \nu}+\right.\right.$ $\left.\left.C_{h \nu}\right)+m_{f}|\Omega|\right\}$, obtemos que

$$
\psi^{\prime}(t) \leq-E(t)-C_{0} \int_{0}^{\infty} \mu^{\prime}(s)\left\|\nabla \eta^{t}(s)\right\|_{2}^{2} d s+C_{0}, \quad t \geq 0 .
$$

Lema 4.8: Dado $E(t)$ como em (3.12), então se cumpre a seguinte igualdade

$$
E^{\prime}(t)=\left\langle T \eta^{t}, \eta^{t}\right\rangle_{\mathcal{M}}=\frac{1}{2} \int_{0}^{\infty} \mu^{\prime}(s)\left\|\nabla \eta^{t}(s)\right\|_{2}^{2} d s .
$$

Demonstração. Notemos que de (3.13) e do Teorema (2.7) temos que

$$
E^{\prime}(t)=\left\langle T \eta^{t}, \eta^{t}\right\rangle_{\mathcal{M}}=\frac{1}{2} \int_{\Omega} \int_{0}^{\infty} \mu^{\prime}(s)\left\|\nabla \eta^{t}(s)\right\|_{\mathbb{R}^{3}}^{2} d s d x=\frac{1}{2} \int_{0}^{\infty} \mu^{\prime}(s)\left\|\nabla \eta^{t}(s)\right\|_{2}^{2} d s,
$$

o que prova o Lema.

\section{Conclusão.}

Pelos Lemas (4.7)-(4.8) temos que

$J^{\prime}(t)=E^{\prime}(t)+\epsilon \psi^{\prime}(t) \leq-\epsilon E(t)+\left(\frac{1}{2}-\epsilon C_{0}\right) \frac{1}{2} \int_{0}^{\infty} \mu^{\prime}(s)\left\|\nabla \eta^{t}(s)\right\|_{2}^{2} d s+\epsilon C_{0}, \quad \epsilon>0$,

alem disso, do Lema (4.6) e tomando $\epsilon \in\left(0, \min \left\{\frac{1}{2 C_{0}}, \epsilon_{0}\right\}\right]$, obtemos 


$$
J^{\prime}(t) \leq-\frac{2 \epsilon}{3} J(t)+\frac{\epsilon C_{h}}{3}+\epsilon C_{0} .
$$

Aplicando a desigualdade de Gronwall acima teremos que

$$
J(t) \leq J(0) e^{-\frac{2 \epsilon t}{3}}+\left(\frac{C_{h} \epsilon}{3}+\epsilon C_{0}\right) \int_{0}^{t} e^{-\frac{2 \epsilon(t-s)}{3}} d s=\left(J(0)-\frac{C_{h}+3 C_{0}}{2}\right) e^{-\frac{2 \epsilon t}{3}}+\frac{C_{h}+3 C_{0}}{2}
$$

Por outro lado, do Lema (4.6) e dado que $\frac{3 C_{0}}{2}>0$ temos

$$
\begin{gathered}
\frac{1}{2} E(t)-\frac{C_{h}}{2} \leq J(t), \\
J(0) \leq \frac{3}{2} E(0)+\frac{C_{h}}{2}+\frac{3 C_{0}}{2},
\end{gathered}
$$

substituindo isto na desigualdade (4.3) se obterá

$$
\frac{1}{2} E(t) \leq \frac{3}{2} E(0) e^{-\frac{2 \epsilon t}{3}}+\frac{2 C_{h}+3 C_{0}}{2} .
$$

Como $\nu \mathcal{E}(t)-C_{h} \leq E(t)$ (vide $\left.(3.17)\right)$, com $\nu>0$ um valor fixo, teremos que

$$
\left\|\left(u(t), u_{t}(t), \eta^{t}\right)\right\|_{\mathscr{H}}^{2}=\mathcal{E}(t) \leq \frac{3}{\nu} E(0) e^{-\frac{2 \epsilon t}{3}}+\frac{3\left(C_{h}+C_{0}\right)}{\nu} .
$$

Com isto, concluímos que qualquer bola fechada de centro em 0 e raio $R$, que denotaremos por $\bar{B}_{R}(0)$, onde $R>\left(1+\frac{3\left(C_{h}+C_{0}\right)}{\nu}\right)^{\frac{1}{2}}$, é um conjunto absorvente limitado do sistema dinâmico $(\mathscr{H}, S(t))$, pois, dado $B \subset \mathscr{H}$ limitado, existe $T_{B}>0$ grande tal que

$$
\frac{3}{\nu} E(0) e^{-\frac{2 \epsilon t}{3}}<1, \quad \forall t>T_{B}
$$

Logo, $S(t) B \in \bar{B}_{R}(0)$ se $t>T_{B}$.

O que prova a Proposição (4.4) e pelo tanto o sistema $(\mathscr{H}, S(t))$ é dissipativo. 


\subsection{Desigualdade de estabilização}

Nesta seção provaremos uma desigualdade de estabilização para duas soluções fracas do sistema (2.17)-(2.18) com diferentes dados inicias. Para a prova construiremos um novo operador baseado no trabalho de Messaoudi \& Tatar [25] e Araujo, Ma \& Qin [2].

Proposição 4.9: Dado um conjunto limitado $B \subset \mathscr{H}$, sejam $z_{1}=\left(u, u_{t}, \eta^{t}\right)$ e $z_{2}=$ $\left(v, v_{t}, \xi^{t}\right)$ duas soluções fracas para o sistema (2.17)-(2.18) tal que $z_{1}(0)=\left(u_{0}, u_{1}, \eta_{0}\right)$ e $z_{2}(0)=\left(v_{0}, v_{1}, \xi_{0}\right)$ pertencem a $B$, então $\left\|z_{1}(t)-z_{2}(t)\right\|_{\mathscr{H}}^{2} \leq C_{B} e^{-\vartheta t}+C_{B} \int_{0}^{t} e^{-\vartheta(t-s)}\left(1+\left\|\nabla u_{t t}(t)\right\|_{2}+\left\|\nabla v_{t t}(t)\right\|_{2}\right)\left(\|w(t)\|_{p+2}+\left\|w_{t}(t)\right\|_{p+2}\right) d s$, onde $w=u-v, \vartheta$ e $C_{B}$ constantes positivas que dependem de $B$, e $p=\max \{\rho, q\}$.

Demonstração. Seja $\zeta^{t}=\eta^{t}-\xi^{t}$, então notemos que o par $(w, \zeta)$ satisfaz o sistema

$$
\begin{gathered}
A w+A w_{t t}+\int_{0}^{\infty} \mu(s) A \zeta^{t}(s) d s=-\left|u_{t}\right|^{\rho} u_{t t}+\left|v_{t}\right|^{\rho} v_{t t}-f(u)+f(v), \\
\zeta_{t}^{t}=-\zeta_{s}^{t}+w_{t}
\end{gathered}
$$

com condições iniciais

$$
w(0)=u(0)-v(0), \quad w_{t}(0)=u_{1}-v_{1}, \quad \zeta^{0}=\eta_{0}-\xi_{0} .
$$

Análogo aos anteriores capítulos, definimos a "Energia Auxiliar" para o sistema (4.4)(4.5) dada por

$$
\mathcal{L}(t)=\frac{1}{2}\|\nabla w(t)\|_{2}^{2}+\frac{1}{2}\left\|\nabla w_{t}(t)\right\|_{2}^{2}+\frac{1}{2}\left\|\zeta^{t}\right\|_{\mathcal{M}}^{2}
$$

Alem disso, definimos o funcional $\Upsilon:[0, \infty) \rightarrow \mathbb{R}$ dado por

$$
\Upsilon(t)=\delta \mathcal{L}(t)+\epsilon \chi(t)+\kappa(t)
$$

onde $\epsilon, \delta>0$ serão fixados posteriormente, e

$$
\begin{gathered}
\chi(t)=\left\langle w_{t}, w\right\rangle_{1} \\
\kappa(t)=-\int_{0}^{\infty} \mu(s)\left\langle w_{t}(t), \zeta^{t}(s)\right\rangle_{1} d s-\int_{0}^{\infty} \mu(s)\left(\frac{1}{\rho+1} \int_{\Omega}\left|u_{t}(t)\right|^{\rho} u_{t}(t) \zeta^{t}(s) d x\right) d s \\
+\int_{0}^{\infty} \mu(s)\left(\frac{1}{\rho+1} \int_{\Omega}\left|v_{t}(t)\right|^{\rho} v_{t}(t) \zeta^{t}(s) d x\right) d s .
\end{gathered}
$$


Observação 4.10: Ao igual que a observação (4.5), as integrais em $\kappa(t)$ são entendidas como dualidade entre $H^{-1}(\Omega)$ e $H_{0}^{1}(\Omega)$.

Lema 4.11: Exitem constantes $\beta_{1}, \beta_{2}, \delta_{0}>0$ tal que

$$
\beta_{1} \mathcal{L}(t) \leq \Upsilon(t) \leq \beta_{2} \mathcal{L}(t), \quad t \geq 0,0<\epsilon \leq \frac{k_{0}}{2}, \delta>\delta_{0}
$$

onde $k_{0}$ é dado pela hipóteses (2.6).

Demonstração. Faremos a prova em duas etapas:

- Estimando $|\epsilon \chi(t)|$

$$
|\chi(t)|=\left|\left\langle w_{t}(t), w(t)\right\rangle_{1}\right| \leq \frac{1}{2}\left\|\nabla w_{t}(t)\right\|_{2}^{2}+\frac{1}{2}\|\nabla w(t)\|_{2}^{2} \leq \mathcal{L}(t),
$$

então para $0<\epsilon \leq \frac{k_{0}}{2}$ temos

$$
|\epsilon \chi(t)| \leq \frac{k_{0}}{2} \mathcal{L}(t)
$$

- Estimando $|\kappa(t)|$

Notemos que ao igual que (4.1), aplicando a desigualdade de Young em (3.18) obtemos que

$$
\left|-\int_{0}^{\infty} \mu(s)\left\langle w_{t}(t), \zeta^{t}(s)\right\rangle_{1} d s\right| \leq \frac{k_{0}}{2}\left\|\nabla w_{t}(t)\right\|_{2}^{2}+\frac{1}{2}\left\|\zeta^{t}\right\|_{\mathcal{M}}^{2} \leq \alpha_{1} \mathcal{L}(t),
$$

$\operatorname{com} \alpha_{1}=\max \left\{1, k_{0}\right\}$.

Por outro lado, observemos que

$$
\begin{gathered}
-\int_{0}^{\infty} \mu(s)\left(\frac{1}{\rho+1} \int_{\Omega}\left|u_{t}(t)\right|^{\rho} u_{t}(t) \zeta^{t}(s) d x\right) d s+\int_{0}^{\infty} \mu(s)\left(\frac{1}{\rho+1} \int_{\Omega}\left|v_{t}(t)\right|^{\rho} v_{t}(t) \zeta^{t}(s) d x\right) d s \\
=-\int_{0}^{\infty} \mu(s) \int_{\Omega}\left(\sigma\left(u_{t}\right)-\sigma\left(v_{t}\right)\right) \zeta^{t}(s) d x d s
\end{gathered}
$$

onde $\sigma$ é a função definida em (3.21).

Por (3.22) temos que 


$$
\begin{aligned}
\left|\int_{0}^{\infty} \mu(s) \int_{\Omega}\left(\sigma\left(u_{t}\right)-\sigma\left(v_{t}\right)\right) \zeta^{t}(s) d x d s\right| \leq & c_{\sigma} \int_{0}^{\infty} \int_{\Omega}\left|u_{t}(t)\right|^{\rho}\left|w_{t}(t) \| \zeta^{t}(s)\right| d x d s \\
& +c_{\sigma} \int_{0}^{\infty} \int_{\Omega}\left|v_{t}(t)\right|^{\rho}\left|w_{t}(t) \| \zeta^{t}(s)\right| d x d s \\
\leq & c_{\sigma} \int_{0}^{\infty} \mu(s)\left\|u_{t}\right\|_{\rho+2}^{\rho}\left\|w_{t}\right\|_{\rho+2}\left\|\zeta^{t}(s)\right\|_{\rho+2} d s \\
& +c_{\sigma} \int_{0}^{\infty} \mu(s)\left\|v_{t}\right\|_{\rho+2}^{\rho}\left\|w_{t}\right\|_{\rho+2}\left\|\zeta^{t}(s)\right\|_{\rho+2} d s \\
\leq & C c_{\sigma} \int_{0}^{\infty} \mu(s)\left\|\nabla u_{t}\right\|_{2}^{\rho}\left\|\nabla w_{t}\right\|_{2}\left\|\nabla \zeta^{t}(s)\right\|_{2} d s \\
& +C c_{\sigma} \int_{0}^{\infty} \mu(s)\left\|\nabla v_{t}\right\|_{2}^{\rho}\left\|\nabla w_{t}\right\|_{2}\left\|\nabla \zeta^{t}(s)\right\|_{2} d s \\
\leq & C c_{\sigma} C_{u h \nu} \int_{0}^{\infty} \mu(s)\left\|\nabla w_{t}\right\|_{2}\left\|\nabla \zeta^{t}(s)\right\|_{2} d s \\
& +C c_{\sigma} C_{v h \nu} \int_{0}^{\infty} \mu(s)\left\|\nabla w_{t}\right\|_{2}\left\|\nabla \zeta^{t}(s)\right\|_{2} d s \\
\leq & C c_{\sigma} C_{u h \nu}\left(\frac{k_{0}}{2}\left\|\nabla w_{t}\right\|_{2}^{2}+\frac{1}{2}\left\|\zeta^{t}\right\|_{\mathcal{M}}^{2}\right) \\
& +C c_{\sigma} C_{v h \nu}\left(\frac{k_{0}}{2}\left\|\nabla w_{t}\right\|_{2}^{2}+\frac{1}{2}\left\|\zeta^{t}\right\|_{\mathcal{M}}^{2}\right) \\
\leq & \alpha_{2} \mathcal{L}(t),
\end{aligned}
$$

onde $C_{u h \nu}$ e $C_{v h \nu}$ são constantes positivas que dependem da $\|h\|_{2}, \nu$ e dos dados iniciais $\left(u_{0}, u_{1}, \eta_{0}\right)$ e $\left(v_{0}, v_{1}, \xi_{0}\right)$ respectivamente (vide (4.3)) e $\alpha_{2}=\max \left\{C c_{\sigma}\left(C_{u h \nu}+\right.\right.$ $\left.\left.C_{v h \nu}\right) \alpha_{1}, 1\right\}$, com $\alpha_{1}$ como na estimativa (4.10).

Observação 4.12: Ao largo do trabalho se usaram as notações $C_{u h \nu}$ e $C_{v h \nu}$ definidas anteriormente.

Finalmente, de esta ultima estimativa e da estimativa (4.9) obtemos

$$
|\Upsilon(t)-\delta \mathcal{L}(t)| \leq\left(\alpha_{2}+\frac{k_{0}}{2}\right) \mathcal{L}(t)
$$

ou seja,

$$
\left(\delta-\alpha_{2}-\frac{k_{0}}{2}\right) \mathcal{L}(t) \leq \Upsilon(t) \leq\left(\delta+\alpha_{2}+\frac{k_{0}}{2}\right) \mathcal{L}(t)
$$

o que prova o lema, pois só basta definir $\delta_{0}=\alpha_{2}+\frac{k_{0}}{2}, \beta_{1}=\delta-\alpha_{2}-\frac{k_{0}}{2}$ e $\beta_{2}=\delta+\alpha_{2}+\frac{k_{0}}{2}$, as quais são constantes positivas para todo $\delta>\delta_{0}$. 
Lema 4.13: Existe uma constante $C_{0}>0$ que depende de $\left(u_{0}, u_{1}, \eta_{0}\right),\left(v_{0}, v_{1}, \xi_{0}\right), \rho, q$ e c, onde c é dada pela hipóteses (2.2), tal que

$$
\mathcal{L}^{\prime}(t) \leq \frac{1}{2} \int_{0}^{\infty} \mu^{\prime}(s)\left\|\nabla \zeta^{t}\right\|_{2}^{2} d s+C_{0}\left(1+\left\|\nabla u_{t t}\right\|_{2}+\left\|\nabla v_{t t}\right\|_{2}\right)\left\|w_{t}\right\|_{p+2}
$$

com $p=\max \{\rho, q\}$.

Demonstração. Notemos que aplicando $w_{t}$ e $\zeta^{t}$ em (4.4) e (4.5) respectivamente, teremos

$$
\begin{gathered}
\left\langle w, w_{t}\right\rangle_{1}+\left\langle w_{t t}, w_{t}\right\rangle_{1}+\int_{0}^{\infty} \mu(s)\left\langle\zeta^{t}(s), w_{t}\right\rangle_{1} d s=\left\langle-\left|u_{t}\right|^{\rho} u_{t t}+\left|v_{t}\right|^{\rho} v_{t t}, w_{t}\right\rangle+\left\langle f(v)-f(u), w_{t}\right\rangle, \\
\left\langle\zeta_{t}^{t}, \zeta^{t}\right\rangle_{\mathcal{M}}=-\left\langle\zeta_{s}^{t}, \zeta^{t}\right\rangle_{\mathcal{M}}+\left\langle w_{t}, \zeta^{t}\right\rangle_{\mathcal{M}},
\end{gathered}
$$

e como

$$
\begin{aligned}
\frac{1}{2} \frac{d}{d t}\|\nabla w(t)\|_{2}^{2} & =\left\langle w(t), w_{t}(t)\right\rangle_{1}, \\
\frac{1}{2} \frac{d}{d t}\left\|\nabla w_{t}(t)\right\|_{2}^{2} & =\left\langle w_{t t}(t), w_{t}(t)\right\rangle_{1}, \\
\frac{1}{2} \frac{d}{d t}\left\|\zeta^{t}\right\|_{\mathcal{M}}^{2} & =\left\langle\zeta_{t}^{t}, \zeta^{t}\right\rangle_{\mathcal{M}},
\end{aligned}
$$

obtemos que

$$
\mathcal{L}^{\prime}(t)=-\left\langle\left|u_{t}(t)\right|^{\rho} u_{t t}(t)-\left|v_{t}(t)\right|^{\rho} v_{t t}(t), w_{t}(t)\right\rangle-\left\langle f(u(t))-f(v(t)), w_{t}(t)\right\rangle-\left\langle\zeta_{s}^{t}, \zeta^{t}\right\rangle_{\mathcal{M}}
$$

Então para provar (4.13) bastara estimar cada termo da igualdade acima

- Estimando $\left|\left\langle\left|u_{t}(t)\right|^{\rho} u_{t t}(t)-\left|v_{t}(t)\right|^{\rho} v_{t t}(t), w_{t}(t)\right\rangle\right|$

$$
\begin{aligned}
\left|\int_{\Omega}\left(\left|u_{t}\right|^{\rho} u_{t t}-\left|v_{t}\right|^{\rho} v_{t t}\right) w_{t} d x\right| & \leq \int_{\Omega}\left|u_{t}\right|^{\rho}\left|u _ { t t } \left\|\left.w_{t}\left|d x+\int_{\Omega}\right| v_{t}\right|^{\rho}\left|v_{t t} \| w_{t}\right| d x\right.\right. \\
& \leq\left\|u_{t}\right\|_{\rho+2}^{\rho}\left\|u_{t t}\right\|_{\rho+2}\left\|w_{t}\right\|_{\rho+2}+\left\|v_{t}\right\|_{\rho+2}^{\rho}\left\|v_{t t}\right\|_{\rho+2}\left\|w_{t}\right\|_{\rho+2} \\
& \leq C\left\|\nabla u_{t}\right\|_{2}^{\rho}\left\|\nabla u_{t t}\right\|_{2}\left\|w_{t}\right\|_{\rho+2}+C\left\|\nabla v_{t}\right\|_{2}^{\rho}\left\|\nabla v_{t t}\right\|_{2}\left\|w_{t}\right\|_{\rho+2} \\
& \leq C C_{u h \nu}\left\|\nabla u_{t t}\right\|_{2}\left\|w_{t}\right\|_{\rho+2}+C C_{v h \nu}\left\|\nabla v_{t t}\right\|_{2}\left\|w_{t}\right\|_{\rho+2} \\
& \leq C_{1}\left(\left\|\nabla u_{t t}\right\|_{2}+\left\|\nabla v_{t t}\right\|_{2}\right)\left\|w_{t}\right\|_{\rho+2},
\end{aligned}
$$

onde $C_{1}=\max \left\{C C_{u h \nu}, C C_{v h \nu}\right\}>0$. 
- Estimando $\left|\left\langle f(u(t))-f(v(t)), w_{t}(t)\right\rangle\right|$

Pelas hipóteses (2.2)-(2.1) e repetindo o processo anterior com $q$ em lugar de $\rho$, teremos que,

$$
\begin{aligned}
\left|\left\langle f(u)-f(v), w_{t}\right\rangle\right| & \leq\left|\left\langle f(u), w_{t}\right\rangle\right|+\left|\left\langle f(v), w_{t}\right\rangle\right| \\
& \leq c \int_{\Omega}\left|u\left\|w_{t}\left|d x+c C C_{u h \nu}\left\|w_{t}\right\|_{q+2}+c \int_{\Omega}\right| v\right\| w_{t}\right| d x+c C C_{v h \nu}\left\|w_{t}\right\|_{q+2} \\
& \leq c\left(\|u\|_{\frac{q+2}{q+1}}\left\|w_{t}\right\|_{q+2}+C C_{u h \nu}\left\|w_{t}\right\|_{q+2}+\|v\|_{\frac{q+2}{q+1}}\left\|w_{t}\right\|_{q+2}+C C_{v h \nu}\left\|w_{t}\right\|_{q+2}\right) \\
& \leq\left(c C\|\nabla u\|_{2}+c C C_{u h \nu}+c C\|\nabla v\|_{2}+c C C_{v h \nu}\right)\left\|w_{t}\right\|_{q+2} \\
& \leq\left(c C C_{u h \nu}+c C C_{u h \nu}+c C C_{v h \nu}+c C C_{v h \nu}\right)\left\|w_{t}\right\|_{q+2} \\
& \leq C_{2}\left\|w_{t}\right\|_{q+2},
\end{aligned}
$$

onde $C_{2}=\max \left\{2 c C C_{u h \nu}, 2 c C C_{v h \nu}\right\}>0$.

Notemos que se utiliza a imersão $H_{0}^{1}(\Omega) \hookrightarrow L^{\frac{q+2}{q+1}}(\Omega)$, a qual é possível dado que $q \in[0,4)$.

- Estimando $-\left\langle\zeta_{s}^{t}, \zeta^{t}\right\rangle_{\mathcal{M}}$

Notemos que pelo Lema (4.8) temos que

$$
-\left\langle\zeta_{s}^{t}, \zeta^{t}\right\rangle_{\mathcal{M}}=\frac{1}{2} \int_{0}^{\infty} \mu^{\prime}(s)\left\|\nabla \zeta^{t}(s)\right\|_{2}^{2} d s
$$

Assim, dadas as estimativas anteriores, comparando com (4.14) teremos que

$$
\mathcal{L}^{\prime}(t) \leq \frac{1}{2} \int_{0}^{\infty} \mu^{\prime}(s)\left\|\nabla \zeta^{t}(s)\right\|_{2}^{2} d s+C_{2}\left\|w_{t}\right\|_{q+2}+C_{1}\left(\left\|\nabla u_{t t}\right\|_{2}+\left\|\nabla v_{t t}\right\|_{2}\right)\left\|w_{t}\right\|_{\rho+2} .
$$

Notemos que tomando $p=\max \{\rho, q\}$, tem-se as imersões $L^{p+2}(\Omega) \hookrightarrow L^{q+2}(\Omega)$ e $L^{p+2}(\Omega) \hookrightarrow L^{\rho+2}(\Omega)$, então

$$
\mathcal{L}^{\prime}(t) \leq \frac{1}{2} \int_{0}^{\infty} \mu^{\prime}(s)\left\|\nabla \zeta^{t}(s)\right\|_{2}^{2} d s+C C_{2}\left\|w_{t}\right\|_{p+2}+C C_{1}\left(\left\|\nabla u_{t t}\right\|_{2}+\left\|\nabla v_{t t}\right\|_{2}\right)\left\|w_{t}\right\|_{p+2}
$$

pelo qual o Lema (4.13) é provado, pois basta definir $C_{0}=\max \left\{C C_{1}, C C_{2}\right\}$.

Lema 4.14: Se cumpre que:

$$
\begin{aligned}
\chi^{\prime}(t) \leq & -\mathcal{L}(t)-\frac{15}{32}\|\nabla w\|_{2}^{2}+\frac{3}{2}\left\|\nabla w_{t}\right\|_{2}^{2}-\frac{16 k_{0}+1}{2 k_{1}} \int_{0}^{\infty} \mu^{\prime}(s)\left\|\nabla \zeta^{t}\right\|_{2}^{2} d s \\
& +C_{0}\left(1+\left\|\nabla u_{t t}\right\|_{2}+\left\|\nabla v_{t t}\right\|_{2}\right)\|w\|_{p+2}
\end{aligned}
$$

sendo $C_{0}>0$ a constante do Lema (4.13) e $p=\max \{\rho, q\}$. 
Demonstração. Notemos que

$$
\chi^{\prime}(t)=\left\langle w_{t t}(t), w(t)\right\rangle_{1}+\left\|\nabla w_{t}(t)\right\|_{2}^{2} .
$$

Aplicando $w$ em (4.4) e comparando com $\chi^{\prime}(t)$, temos que $\chi^{\prime}(t)=-\int_{0}^{\infty} \mu(s)\left\langle\zeta^{t}(s), w\right\rangle_{1} d s-\left\langle\left|u_{t}\right|^{\rho} u_{t t}-\left|v_{t}\right|^{\rho} v_{t t}, w\right\rangle-\langle f(u)-f(v), w\rangle-\|\nabla w\|_{2}^{2}+\left\|\nabla w_{t}\right\|_{2}^{2}$,

agora, razonando igual que nas estimativas feitas no Lema (4.13), teremos

$\chi^{\prime}(t) \leq-\int_{0}^{\infty} \mu(s)\left\langle\zeta^{t}(s), w\right\rangle_{1} d s+C_{1}\left(\left\|\nabla u_{t t}\right\|_{2}+\left\|\nabla v_{t t}\right\|_{2}\right)\|w\|_{\rho+2}+C_{2}\|w\|_{q+2}-\|\nabla w\|_{2}^{2}+\left\|\nabla w_{t}\right\|_{2}^{2}$,

alem disso, procedendo igual que (4.1) e tomando $\epsilon=8$ na desigualdade de Young, obtemos

$\chi^{\prime}(t) \leq 8 k_{0}\left\|\zeta^{t}\right\|_{\mathcal{M}}^{2}+\frac{1}{32}\|\nabla w\|_{2}^{2}+C_{1}\left(\left\|\nabla u_{t t}\right\|_{2}+\left\|\nabla v_{t t}\right\|_{2}\right)\|w\|_{\rho+2}+C_{2}\|w\|_{q+2}-\|\nabla w\|_{2}^{2}+\left\|\nabla w_{t}\right\|_{2}^{2}$.

Por tanto, somando e restando $\mathcal{L}(t)$ na expressão anterior

$\chi^{\prime}(t) \leq-\mathcal{L}(t)+\left(8 k_{0}+\frac{1}{2}\right)\left\|\zeta^{t}\right\|_{\mathcal{M}}^{2}-\frac{15}{32}\|\nabla w\|_{2}^{2}+C_{0}\left(1+\left\|\nabla u_{t t}\right\|_{2}+\left\|\nabla v_{t t}\right\|_{2}\right)\|w\|_{p+2}+\frac{3}{2}\left\|\nabla w_{t}\right\|_{2}^{2}$,

onde $p=\max \{\rho, q\}, C_{0}=\max \left\{C C_{1}, C C_{2}\right\}$ sendo $C>0$ a constante das imersões $L^{p+2}(\Omega) \hookrightarrow L^{q+2}(\Omega)$ e $L^{p+2}(\Omega) \hookrightarrow L^{\rho+2}(\Omega)$, isto é, a mesma constante do Lema (4.13).

Finalmente, pela hipóteses (2.7) teremos,

$$
\begin{aligned}
\chi^{\prime}(t) \leq & -\mathcal{L}(t)-\frac{15}{32}\|\nabla w\|_{2}^{2}+\frac{3}{2}\left\|\nabla w_{t}\right\|_{2}^{2}-\frac{16 k_{0}+1}{2 k_{1}} \int_{0}^{\infty} \mu^{\prime}(s)\left\|\nabla \zeta^{t}\right\|_{2}^{2} d s \\
& +C_{0}\left(1+\left\|\nabla u_{t t}\right\|_{2}+\left\|\nabla v_{t t}\right\|_{2}\right)\|w\|_{p+2}
\end{aligned}
$$

o que prova o Lema.

Lema 4.15: Existe uma constante $C_{\kappa}>0$ que depende de $\left(u_{0}, u_{1}, \eta_{0}\right),\left(v_{0}, v_{1}, \xi_{0}\right), \rho, q$, $c, k_{0}$ e $k_{1}$, onde c é dada pela hipóteses (2.2) e $k_{0}, k_{1}$ são dados pelas hipóteses (2.6) e (2.7), tal que

$$
\kappa^{\prime}(t) \leq \frac{k_{0}}{8}\|\nabla w\|_{2}^{2}-\frac{7 k_{0}}{8}\left\|\nabla w_{t}\right\|_{2}^{2}-C_{\kappa} \int_{0}^{\infty} \mu^{\prime}(s)\left\|\nabla \zeta^{t}(s)\right\|_{2}^{2} d s
$$

Demonstração. Pela definição de $\kappa(t)$ (vide (4.8)), temos que

$$
\kappa^{\prime}(t)=A(t)+B(t)
$$


onde

$$
\begin{gathered}
A(t):=\int_{\Omega}\left(\left|v_{t}(t)\right|^{\rho} v_{t t}(t)-\left|u_{t}(t)\right|^{\rho} u_{t t}(t)-A w_{t t}(t)\right)\left(\int_{0}^{\infty} \mu(s) \zeta^{t}(s) d s\right) d x, \\
B(t):=\int_{\Omega}\left(\frac{1}{\rho+1}\left|v_{t}(t)\right|^{\rho} v_{t}(t)-\frac{1}{\rho+1}\left|u_{t}(t)\right|^{\rho} u_{t}(t)-A w_{t}(t)\right)\left(\int_{0}^{\infty} \mu(s) \zeta_{t}^{t}(s) d s\right) d x .
\end{gathered}
$$

Para provar o Lema, estimaremos $A(t)$ e $B(t)$ por separado.

- Estimando $A(t)$

Substituindo (4.4) em (4.16) obtemos

$$
A(t)=\int_{\Omega}\left(A w+\int_{0}^{\infty} \mu(s) A \zeta(s) d s+f(u)-f(v)\right)\left(\int_{0}^{\infty} \mu(s) \zeta^{t}(s) d s\right) d x .
$$

Estimemos termo por termo da igualdade acima.

- Estimando $\int_{\Omega} A w\left(\int_{0}^{\infty} \mu(s) \zeta^{t}(s) d s\right) d x$

$$
\int_{\Omega} A w\left(\int_{0}^{\infty} \mu(s) \zeta^{t}(s) d s\right) d x=\int_{0}^{\infty} \mu(s)\left\langle w, \zeta^{t}(s)\right\rangle_{1} d s \leq \frac{k_{0}}{32}\|\nabla w\|_{2}^{2}+8\left\|\zeta^{t}\right\|_{\mathcal{M}}^{2}
$$

Para a ultima desigualdade se razona igual quem (4.1), usando a desigualdade de Young com $\epsilon=8$ para os termos $k_{0}^{\frac{1}{2}}\|\nabla w\|_{2}$ e $\left\|\zeta^{t}\right\|$.

- Estimando $\int_{\Omega}\left(\int_{0}^{\infty} \mu(s) A \zeta(s) d s\right)\left(\int_{0}^{\infty} \mu(s) \zeta^{t}(s) d s\right) d x$

$$
\begin{aligned}
\int_{\Omega}\left(\int_{0}^{\infty} \mu(s) A \zeta(s) d s\right) & \left(\int_{0}^{\infty} \mu(s) \zeta^{t}(s) d s\right) d x=\int_{0}^{\infty} \mu(s)\left(\int_{0}^{\infty} \mu(y)\left\langle\zeta^{t}(s), \zeta^{t}(y)\right\rangle_{1} d y\right) d s \\
& \leq \int_{0}^{\infty} \mu(s)\left(\int_{0}^{\infty} \mu(y)\left\|\nabla \zeta^{t}(s)\right\|_{2}\left\|\nabla \zeta^{t}(y)\right\|_{2} d y\right) d s \\
& =\int_{0}^{\infty} \mu(s)\left\|\nabla \zeta^{t}(s)\right\|_{2}\left(\int_{0}^{\infty} \mu(y)\left\|\nabla \zeta^{t}(y)\right\|_{2} d y\right) d s \\
& \leq k_{0}^{\frac{1}{2}} \int_{0}^{\infty} \mu(s)\left\|\nabla \zeta^{t}(s)\right\|_{2}\left(\int_{0}^{\infty} \mu(y)\left\|\nabla \zeta^{t}(y)\right\|_{2}^{2} d y\right)^{\frac{1}{2}} d s \\
& =k_{0}^{\frac{1}{2}} \int_{0}^{\infty} \mu(s)\left\|\nabla \zeta^{t}(s)\right\|_{2}\left\|\zeta^{t}\right\|_{\mathcal{M}} d s \\
& \leq k_{0}\left\|\zeta^{t}\right\|_{\mathcal{M}}^{2} .
\end{aligned}
$$


- Estimando $\int_{\Omega}(f(u)-f(v))\left(\int_{0}^{\infty} \mu(s) \zeta^{t}(s) d s\right) d x$

Pela hipóteses (2.2) se tem que

$$
\int_{\Omega}(f(u)-f(v))\left(\int_{0}^{\infty} \mu(s) \zeta^{t}(s) d s\right) d x \leq c \int_{\Omega}\left(1+|u|^{q}+|v|^{q}\right)|w|\left(\int_{0}^{\infty} \mu(s) \zeta^{t}(s) d s\right) d x .
$$

Notemos que

$$
\begin{aligned}
c \int_{\Omega}|w|\left(\int_{0}^{\infty} \mu(s) \zeta^{t}(s) d s\right) d x & =c \int_{0}^{\infty} \mu(s)\left(\int_{\Omega}|w| \zeta^{t}(s) d x\right) d s \\
& \leq c \int_{0}^{\infty} \mu(s)\|w\|_{2}\|\zeta(s)\|_{2} d s \\
& \leq \frac{c}{\lambda_{1}^{2}} \int_{0}^{\infty} \mu(s)\|\nabla w\|_{2}\|\nabla \zeta(s)\|_{2} d s \\
& =\frac{c}{\lambda_{1}^{2}}\|\nabla w\|_{2} \int_{0}^{\infty} \mu(s)\|\nabla \zeta(s)\|_{2} d s \\
& \leq \frac{c k_{0}^{\frac{1}{2}}}{\lambda_{1}^{2}}\|\nabla w\|_{2}\|\zeta\|_{\mathcal{M}} \\
& \leq \frac{c^{2}}{\lambda_{1}^{4}} \frac{k_{0}}{4 \epsilon}\|\nabla w\|_{2}^{2}+\epsilon\|\zeta\|_{\mathcal{M}}^{2} \\
& =\frac{k_{0}}{32}\|\nabla w\|_{2}^{2}+\frac{8 c^{2}}{\lambda_{1}^{4}}\left\|\zeta^{t}\right\|_{\mathcal{M}}^{2}, \quad \epsilon=8 .
\end{aligned}
$$

e

$$
\begin{aligned}
c \int_{\Omega}|u|^{q}\left(\int_{0}^{\infty} \mu(s) \zeta^{t}(s) d s\right) d x & =c \int_{0}^{\infty} \mu(s)\left(\int_{\Omega}|u|^{q} \zeta^{t}(s) d x\right) d s \\
& \leq c \int_{0}^{\infty} \mu(s)\|u\|_{q+2}^{q}\|w\|_{q+2}\|\zeta(s)\|_{q+2} d s \\
& \leq c C C_{u h \nu} \int_{0}^{\infty} \mu(s)\|\nabla w\|_{2}\|\nabla \zeta(s)\|_{2} d s \\
& \leq k_{0} c^{2} C^{2} C_{u h \nu} \frac{1}{4 \epsilon}\|\nabla w\|_{2}^{2}+\epsilon\left\|\zeta^{t}\right\|_{\mathcal{M}} \\
& =\frac{k_{0}}{32}\|\nabla w\|_{2}^{2}+8 C_{3}\left\|\zeta^{t}\right\|_{\mathcal{M}}, \quad \epsilon=8 c^{2} C^{2} C_{u h \nu}
\end{aligned}
$$

onde $C_{3}=c^{2} C^{2} C_{u h \nu}>0$.

De maneira análoga, existira uma constante $C_{4}=c^{2} C^{2} C_{v h \nu}>0$ tal que

$$
c \int_{\Omega}|v|^{q}\left(\int_{0}^{\infty} \mu(s) \zeta^{t}(s) d s\right) d x \leq \frac{k_{0}}{32}\|\nabla w\|_{2}^{2}+8 C_{4}\left\|\zeta^{t}\right\|_{\mathcal{M}} .
$$

Ou seja, 


$$
\int_{\Omega}(f(u)-f(v))\left(\int_{0}^{\infty} \mu(s) \zeta^{t}(s) d s\right) d x \leq \frac{3 k_{0}}{32}\|\nabla w\|_{2}^{2}+8 C_{5}\left\|\zeta^{t}\right\|_{\mathcal{M}}^{2}
$$

onde $C_{5}=\frac{c^{2}}{\lambda_{1}^{4}}+C_{3}+C_{4}>0$.

Assim temos que

$$
A(t) \leq \frac{k_{0}}{8}\|\nabla w\|_{2}^{2}+\left(k_{0}+8 C_{5}+8\right)\left\|\zeta^{t}\right\|_{\mathcal{M}}^{2}
$$

mas, como

$$
\left\|\zeta^{t}\right\|_{\mathcal{M}}^{2}=\int_{0}^{\infty} \mu(s)\left\|\nabla \zeta^{t}\right\|_{2}^{2} d s \leq-\frac{1}{k_{1}} \int_{0}^{\infty} \mu^{\prime}(s)\left\|\nabla \zeta^{t}(s)\right\|_{2} d s
$$

então poderemos estimar $A(t)$ por

$$
A(t) \leq \frac{k_{0}}{8}\|\nabla w\|_{2}^{2}-\frac{k_{0}+8 C_{5}+8}{k_{1}} \int_{0}^{\infty} \mu^{\prime}(s)\left\|\nabla \zeta^{t}(s)\right\|_{2} d s .
$$

\section{- Estimando $B(t)$}

Notemos que pela equação (4.5) temos que

$$
\int_{0}^{\infty} \mu(s) \zeta_{t}^{t}(s) d s=\int_{0}^{\infty} \mu^{\prime}(s) \zeta^{t}(s) d s+k_{0} w_{t}(t)
$$

Assim, $B(t)$ se pode expressar como

$$
B(t)=C(t)+k_{0} D(t)
$$

onde

$$
\begin{gathered}
C(t)=\int_{\Omega}\left(\frac{1}{\rho+1}\left|v_{t}(t)\right|^{\rho} v_{t}(t)-\frac{1}{\rho+1}\left|u_{t}(t)\right|^{\rho} u_{t}(t)-A w_{t}(t)\right)\left(\int_{0}^{\infty} \mu^{\prime}(s) \zeta^{t}(s) d s\right) d x \\
D(t)=\int_{\Omega}\left(\frac{1}{\rho+1}\left|v_{t}(t)\right|^{\rho} v_{t}(t)-\frac{1}{\rho+1}\left|u_{t}(t)\right|^{\rho} u_{t}(t)-A w_{t}(t)\right) w_{t} d x
\end{gathered}
$$

Estimaremos $C(t)$ e $D(t)$. 


\section{- Estimando $C(t)$}

Notemos que pelo feito quando se estimo $|\kappa(t)|$, pelas hipóteses $(2.6),(2.7)$ e (2.8), e pela desigualdade de Young, temos que

$$
\begin{aligned}
& \int_{\Omega}\left(\frac{\left|v_{t}\right|^{\rho} v_{t}}{\rho+1}-\frac{\left|u_{t}\right|^{\rho} u_{t}}{\rho+1}\right)\left(\int_{0}^{\infty} \mu^{\prime}(s) \zeta^{t} d s\right) d x=-\int_{0}^{\infty} \mu^{\prime}(s) \int_{\Omega}\left(\sigma\left(u_{t}\right)-\sigma\left(v_{t}\right)\right) \zeta^{t}(s) d x d s \\
& \leq-C c_{\sigma} C_{u h \nu} \int_{0}^{\infty} \mu^{\prime}(s)\left\|\nabla w_{t}\right\|_{2}\left\|\nabla \zeta^{t}(s)\right\|_{2} d s \\
&-C c_{\sigma} C_{v h \nu} \int_{0}^{\infty} \mu^{\prime}(s)\left\|\nabla w_{t}\right\|_{2}\left\|\nabla \zeta^{t}(s)\right\|_{2} d s \\
& \leq C c_{\sigma} C_{u h \nu} k_{2}^{\frac{1}{2}}\left\|\nabla w_{t}\right\|_{2}\left(\int_{0}^{\infty}-\mu^{\prime}(s)\left\|\nabla \zeta^{t}(s)\right\|_{2}^{2} d s\right)^{\frac{1}{2}} \\
&+C c_{\sigma} C_{v h \nu} k_{2}^{\frac{1}{2}}\left\|\nabla w_{t}\right\|_{2}\left(\int_{0}^{\infty}-\mu^{\prime}(s)\left\|\nabla \zeta^{t}(s)\right\|_{2}^{2} d s\right)^{\frac{1}{2}} \\
& \leq C c_{\sigma} C_{u h \nu} k_{2}^{\frac{1}{2}}\left\|\nabla w_{t}\right\|_{2}\left(\int_{0}^{\infty}-\mu^{\prime}(s)\left\|\nabla \zeta^{t}(s)\right\|_{2}^{2} d s\right)^{\frac{1}{2}} \\
&+C c_{\sigma} C_{v h \nu} k_{2}^{\frac{1}{2}}\left\|\nabla w_{t}\right\|_{2}\left(\int_{0}^{\infty}-\mu^{\prime}(s)\left\|\nabla \zeta^{t}(s)\right\|_{2}^{2} d s\right)^{\frac{1}{2}} \\
& \leq \frac{k_{0}}{32}\left\|\nabla w_{t}\right\|_{2}^{2}-\frac{\epsilon_{1}^{u} k_{2}}{k_{0}}\left(\int_{0}^{\infty} \mu^{\prime}(s)\left\|\nabla \zeta^{t}(s)\right\|_{2}^{2} d s\right) \\
&+\frac{1}{32}\left\|\nabla w_{t}\right\|_{2}^{2}-\frac{\epsilon_{1}^{v} k_{2}}{k_{0}}\left(\int_{0}^{\infty} \mu^{\prime}(s)\left\|\nabla \zeta^{t}(s)\right\|_{2}^{2} d s\right) \\
&= \frac{k_{0}}{16}\left\|\nabla w_{t}\right\|_{2}^{2}-\left(\frac{\epsilon_{1}^{u} k_{2}+\epsilon_{1}^{v} k_{2}}{k_{0}}\right)\left(\int_{0}^{\infty} \mu^{\prime}(s)\left\|\nabla \zeta^{t}(s)\right\|_{2}^{2} d s\right), \\
&
\end{aligned}
$$

onde $\epsilon_{1}^{u}=8 C^{2} c_{\sigma}^{2} C_{u h \nu}$ e $\epsilon_{1}^{v}=8 C^{2} c_{\sigma}^{2} C_{v h \nu}$.

Alem disso, se cumpre que

$$
\begin{aligned}
\int_{\Omega}-A w_{t}\left(\int_{0}^{\infty} \mu^{\prime}(s) \zeta^{t}(s) d s\right) d x & =-\int_{0}^{\infty} \mu^{\prime}(s)\left\langle w_{t}, \zeta^{t}(s)\right\rangle_{1} d s \\
& \leq \frac{k_{0}}{16}\left\|\nabla w_{t}\right\|_{2}^{2}-\frac{4 k_{2}}{k_{0}} \int_{0}^{\infty} \mu^{\prime}(s)\left\|\nabla \zeta^{t}(s)\right\|_{2}^{2} d s
\end{aligned}
$$

Pelo qual se obtêm que

$$
C(t) \leq \frac{k_{0}}{8}\left\|\nabla w_{t}\right\|_{2}^{2}-\left(\frac{\epsilon_{1}^{u} k_{2}+\epsilon_{1}^{v} k_{2}+4 k_{2}}{k_{0}}\right) \int_{0}^{\infty} \mu^{\prime}(s)\left\|\nabla \zeta^{t}(s)\right\|_{2}^{2} d s
$$


- Estimando $D(t)$

Notemos que $D(t)$ se pode escrever como

$$
D(t)=-\int_{\Omega}\left(\sigma\left(u_{t}(t)\right)-\sigma\left(v_{t}(t)\right)\right)\left(u_{t}(t)-v_{t}(t)\right) d x-\left\|\nabla w_{t}(t)\right\|_{2}^{2},
$$

mas como $\sigma$ é monótona (vide (3.13)), isto é,

$$
\left(\sigma\left(u_{t}(t)\right)-\sigma\left(v_{t}(t)\right)\right)\left(u_{t}(t)-v_{t}(t)\right) \geq 0
$$

então,

$$
D(t) \leq-\left\|\nabla w_{t}(t)\right\|_{2}^{2}
$$

Substituindo (4.20)-(4.21) em (4.19) obtemos

$$
B(t) \leq-\frac{7 k_{0}}{8}\left\|\nabla w_{t}\right\|_{2}^{2}-\left(\frac{\epsilon_{1}^{u} k_{2}+\epsilon_{1}^{v} k_{2}+4 k_{2}}{k_{0}}\right) \int_{0}^{\infty} \mu^{\prime}(s)\left\|\nabla \zeta^{t}(s)\right\|_{2}^{2} d s .
$$

Finalmente, substituindo (4.18)-(4.22) em (4.15) obtém-se que

$$
\kappa^{\prime}(t) \leq-\frac{7 k_{0}}{8}\left\|\nabla w_{t}\right\|_{2}^{2}+\frac{k_{0}}{8}\|\nabla w\|_{2}^{2}-C_{\kappa} \int_{0}^{\infty} \mu^{\prime}(s)\left\|\nabla \zeta^{t}(s)\right\|_{2}^{2} d s,
$$

onde, $C_{\kappa}=\frac{k_{0}+8 C_{5}+8}{k_{1}}+\epsilon_{1}^{u}+\epsilon_{1}^{v}+4>0$, pois pela observação $(2.1)$, temos que

$$
\frac{k_{0}+8 C_{5}+8}{k_{1}}+\left(\frac{\epsilon_{1}^{u} k_{2}+\epsilon_{1}^{v} k_{2}+4 k_{2}}{k_{0}}\right) \leq \frac{k_{0}+8 C_{5}+8}{k_{1}}+\epsilon_{1}^{u}+\epsilon_{1}^{v}+4=C_{\kappa} .
$$

\section{Conclusão.}

Dados os Lemas (4.13), (4.14) e (4.15), e pela definição de $\Upsilon(t)$, temos que

$$
\begin{aligned}
\Upsilon^{\prime}(t) \leq & \left(\frac{\delta}{2}-\frac{16 k_{0}+1}{2 k_{1}} \epsilon-C_{\kappa}\right) \int_{0}^{\infty} \mu^{\prime}(s)\left\|\nabla \zeta^{t}(s)\right\|_{2}^{2} d s+\left(\frac{k_{0}}{8}-\frac{15 \epsilon}{32}\right)\|\nabla w\|_{2}^{2} \\
& +\left(\frac{3 \epsilon}{2}-\frac{7 k_{0}}{8}\right)\left\|\nabla w_{t}\right\|_{2}^{2}-\epsilon \mathcal{L}(t)+C_{0}\left(1+\left\|\nabla u_{t t}\right\|_{2}+\left\|\nabla v_{t t}\right\|_{2}\right)\left(\delta\left\|w_{t}\right\|_{p+2}+\epsilon\|w\|_{p+2}\right) .
\end{aligned}
$$

Tomando 


$$
\begin{aligned}
& \frac{4 k_{0}}{15}<\epsilon<\frac{k_{0}}{2}<\frac{7 k_{0}}{12}, \\
& \delta>\frac{16 k_{0}+1}{k_{1}} \epsilon+2 C_{\kappa},
\end{aligned}
$$

a expressão acima reduz-se a

$\Upsilon^{\prime}(t) \leq-\epsilon \mathcal{L}(t)+C_{\Upsilon}\left(1+\left\|\nabla u_{t t}\right\|_{2}+\left\|\nabla v_{t t}\right\|_{2}\right)\left(\left\|w_{t}\right\|_{p+2}+\|w\|_{p+2}\right), \quad \forall \epsilon \in\left(\frac{4 k_{0}}{15}, \frac{k_{0}}{2}\right), \forall \delta>\delta_{\Upsilon}$, onde $\delta_{\Upsilon}=\max \left\{\delta_{0}, \frac{16 k_{0}+1}{k_{1}} \epsilon+2 C_{\kappa}\right\}$, com $\delta_{0}$ definido no Lema (4.11) e $C_{\Upsilon}=C_{0} \max \{\epsilon, \delta\}$.

Observação 4.16: Notemos que é as restrições $\epsilon \in\left(\frac{4 k_{0}}{15}, \frac{k_{0}}{2}\right)$ e $\delta>\delta_{\Upsilon}$, nos permite usar o Lema (4.11) para algum $\epsilon$ e $\delta$ fixo.

Denotemos por

$$
m(t):=C_{\Upsilon}\left(1+\left\|\nabla u_{t t}\right\|_{2}+\left\|\nabla v_{t t}\right\|_{2}\right)\left(\left\|w_{t}\right\|_{p+2}+\|w\|_{p+2}\right),
$$

assim pelo Lema (4.11) temos que

$$
\Upsilon^{\prime}(t) \leq \frac{\epsilon}{\beta_{2}} \Upsilon(t)+m(t)
$$

e pela desigualdade de Gronwall obtemos

$$
\Upsilon(t) \leq \Upsilon(0) e^{-\frac{\epsilon t}{\beta_{2}}}+\int_{0}^{t} e^{-\frac{\epsilon(t-s)}{\beta_{2}}} m(s) d s
$$

Novamente pelo Lema (4.11) temos

$$
\mathcal{L}(t) \leq \frac{\beta_{2}}{\beta_{1}} \mathcal{L}(0) e^{-\frac{\epsilon t}{\beta_{2}}}+\frac{1}{\beta_{1}} \int_{0}^{t} e^{-\frac{\epsilon(t-s)}{\beta_{2}}} m(s) d s
$$

ou seja,

$$
\left\|z_{1}(t)-z_{2}(t)\right\|_{\mathscr{H}}^{2} \leq C_{B} e^{-\vartheta t}+C_{B} \int_{0}^{t} e^{-\vartheta(t-s)} m(s) d s
$$

$\operatorname{com} C_{B}=\max \left\{\frac{\beta_{2}}{\beta_{1}} \mathcal{L}(0), \frac{1}{\beta_{1}}\right\}>0$ e $\vartheta=\frac{\epsilon}{\beta_{2}}$ para algum $\epsilon \in\left(\frac{4 k_{0}}{15}, \frac{k_{0}}{2}\right)$ e $\delta>\delta_{\Upsilon}$. 


\subsection{Compacidade assintótica}

Proposição 4.17: O sistema dinâmico ( $\mathscr{H}, S(t))$ correspondente ao sistema (2.17)(2.18) é assintoticamente compacto.

Demonstração. Para demostrar o Teorema, começaremos provando que o sistema dinâmico $(\mathscr{H}, S(t))$ é assintoticamente regular, para isto usaremos os Teoremas 1.31 e 1.17.

Seja $B$ um subconjunto limitado de $\mathscr{H}$ e positivamente invariante com respeito a $S(t)$. Dado $\epsilon>0$, tomemos $\tau>0$, tal que

$$
C_{B}^{\frac{1}{2}} e^{-\frac{\vartheta \tau}{2}}<\epsilon
$$

onde $C_{B}$ e $\vartheta$ são dados pela Proposição (4.9)

Lema 4.18: Existe uma constante $C_{B, \tau}>0$ tal que se cumpre

$$
\left\|S(\tau) z_{0}^{1}-S(\tau) z_{0}^{2}\right\|_{\mathscr{H}} \leq \epsilon+\phi_{\tau}\left(z_{0}^{1}, z_{0}^{2}\right), \quad \forall z_{0}^{1}, z_{0}^{2} \in B
$$

onde

$$
\phi_{\tau}\left(z_{0}^{1}, z_{0}^{2}\right)=C_{B, \tau}\left(\int_{0}^{\tau}\|u(s)-v(s)\|_{p+2}^{2}+\left\|u_{t}(s)-v_{t}(s)\right\|_{p+2}^{2} d s\right)^{\frac{1}{4}}
$$

$\operatorname{com} p=\max \{\rho, q\}$.

Demonstração. Consideremos $z_{1}(t)=\left(u(t), u_{t}(t), \eta^{t}\right)=S(t) z_{0}^{1}$ e $z_{2}(t)=\left(v(t), v_{t}(t), \xi^{t}\right)=$ $S(t) z_{0}^{2}$ soluções fracas do sistema (2.17)-(2.18) com respeito aos dados inicias $z_{0}^{1}, z_{0}^{2} \in B$.

Então, no contexto da Proposição (4.9), para todo $t \in(0, \tau)$ temos que,

$$
\begin{aligned}
\| z_{1}(\tau) & -z_{2}(\tau) \|_{\mathscr{H}}^{2} \leq C_{B} e^{-\vartheta \tau}+C_{B} \int_{0}^{\tau} e^{-\vartheta(\tau-s)}\left(1+\left\|\nabla u_{t t}\right\|_{2}+\left\|\nabla v_{t t}\right\|_{2}\right)\left(\|w\|_{p+2}+\left\|w_{t}\right\|_{p+2}\right) d s \\
& \leq C_{B} e^{-\vartheta \tau}+2^{2} C_{B}\left(\int_{0}^{\tau} e^{-\vartheta(\tau-s)}\left(1+\left\|\nabla u_{t t}\right\|_{2}+\left\|\nabla v_{t t}\right\|_{2}\right)\right)^{\frac{1}{2}}\left(\int_{0}^{\tau}\|w\|_{p+2}+\left\|w_{t}\right\|_{p+2}\right)^{\frac{1}{2}} .
\end{aligned}
$$

Agora, como $u_{t t}$ e $v_{t t}$ são limitados em $L^{2}\left(0, \tau ; H_{0}^{1}(\Omega)\right)$, então existe uma constante $C_{\mathcal{B}, \tau}>0$ tal que

$$
\left\|z_{1}(\tau)-z_{2}(\tau)\right\|_{\mathscr{H}}^{2} \leq C_{B} e^{-\vartheta \tau}+C_{B, \tau}^{2}\left(\int_{0}^{\tau}\|w\|_{p+2}+\left\|w_{t}\right\|_{p+2}\right)^{\frac{1}{2}},
$$


então,

$$
\begin{aligned}
\left\|z_{1}(\tau)-z_{2}(\tau)\right\|_{\mathscr{H}} & \leq C_{B}^{\frac{1}{2}} e^{-\frac{\vartheta \tau}{2}}+C_{B, \tau}\left(\int_{0}^{\tau}\|w\|_{p+2}+\left\|w_{t}\right\|_{p+2}\right)^{\frac{1}{4}} \\
& \leq \epsilon+C_{B, \tau}\left(\int_{0}^{\tau}\|w\|_{p+2}+\left\|w_{t}\right\|_{p+2}\right)^{\frac{1}{4}}
\end{aligned}
$$

para algum $\epsilon>0$ que cumpra (4.23).

Lema 4.19: No contexto do Lema anterior, se cumpre que

$$
\liminf _{n \rightarrow \infty} \liminf _{m \rightarrow \infty} \phi_{\tau}\left(z^{n}, z^{m}\right)=0
$$

para alguma sequencia $\left(z^{n}\right) \subset B$.

Demonstração. Seja $\left(z_{0}^{n}\right)=\left(u_{0}^{n}, v_{0}^{n}, \eta_{0}^{n}\right)_{n \in \mathbb{N}}$ uma sequência em $B$.

Consideremos $S(t)\left(z_{0}^{n}\right)=\left(u^{n}(t), u_{t}^{n}(t), \eta^{t, n}\right)_{n \in \mathbb{N}}$, como $B$ é positivamente invariante pela ação de $S(t)$ então $\left(u^{n}(t), u_{t}^{n}(t), \eta^{t, n}\right)_{n \in \mathbb{N}}$ é uniformemente limitada em $\mathscr{H}$, em particular temos que

$$
\left(u^{n}\right),\left(u_{t}^{n}\right) \text { são limitadas em } C\left([0, \infty) ; H_{0}^{1}(\Omega)\right) .
$$

Agora, como $\left(u_{t}^{n}\right)$ é limitada em $C\left([0, \infty) ; H_{0}^{1}(\Omega)\right)$, então

$$
\left(u_{t t}^{n}\right) \text { é limitada em } L^{2}\left(0, \tau ; L^{2}(\Omega)\right), \quad \forall \tau>0,
$$

assim teremos que

$$
\left(u_{t}^{n}\right),\left(u_{t t}^{n}\right) \text { são limitadas em } L^{2}\left(0, \tau ; L^{2}(\Omega)\right) .
$$

Agora como se tem a cadeia de imersões

$$
H_{0}^{1}(\Omega) \stackrel{c}{\hookrightarrow} L^{p+2}(\Omega) \hookrightarrow L^{2}(\Omega), \quad p=\max \{\rho, q\}
$$

tomando respectivamente no Teorema 1.17

$$
X=H_{0}^{1}(\Omega), \quad B=L^{p+2}(\Omega), \quad Y=L^{2}(\Omega), \quad r=2,
$$

temos que $\left(u^{n}\right)$ e $\left(u_{t}^{n}\right)$ são relativamente compactos em $C\left(0, \tau ; H_{0}^{1}(\Omega)\right)$ para todo $\tau>0$.

Passando a uma subsequencia se for necessário em cada casso, as quais denotaremos igualmente, teremos que

$$
\left(u^{n}\right),\left(u_{t}^{n}\right) \text { convergem (fortemente) em } C\left(0, \tau ; L^{p+2}(\Omega)\right), \quad \forall \tau>0,
$$


podemos concluir assim que

$$
\lim _{n \rightarrow \infty} \lim _{m \rightarrow \infty} C_{B, \tau}\left(\int_{0}^{\tau}\left\|u^{n}(s)-u^{m}(s)\right\|_{p+2}^{2}+\left\|u_{t}^{n}(s)-u_{t}^{m}(s)\right\|_{p+2}^{2} d s\right)^{\frac{1}{4}}=0
$$

o que demonstra o Lema.

\section{Conclusão.}

Pelo Teorema 1.31 e pelos Lemas (4.18)-(4.19), concluímos que o sistema dinâmico $(\mathscr{H}, S(t))$ correspondente ao sistema (2.17)-(2.18) é assintoticamente regular e pela Proposição (4.4) também é dissipativo, então temos como resultado do Teorema 1.30 que o sistema dinâmico é assintoticamente compacto o que prova a proposição.

\section{Conclusão do Teorema 4.2.}

Notemos que pela Proposição (4.4) temos que o sistema dinâmico $(\mathscr{H}, S(t))$ é dissipativo, também notemos que pela Proposição (4.17) temos que o sistema dinâmico $(\mathscr{H}, S(t))$ é assintoticamente compacto.

Assim pelo Teorema 1.28, concluímos que o sistema dinâmico $(\mathscr{H}, S(t))$ possui atrator global. 


\section{Referências Bibliográficas}

[1] R. A. Adams and J. J. F. Fournier, Sobolev Spaces, second edition, Pure and Applied Mathematics (Amsterdam), 140. Elsevier/Academic Press (2003).

[2] R. O. Araújo, T. F. Ma and Y. Qin, Long-time behavior of a quasilinear viscoelastic equation with past history, Journal of Differential Equations 254 (2013), 4066-4087.

[3] A. V. Babin and M. I. Vishik, Attractors of Evolution Equations, Studies in Mathematics and its Applications 25, North-Holland, Amsterdam (1992).

[4] H. Brézis, Analyse Fonctionnelle: Théorie et Applications, Paris, Masson (1983).

[5] P. L. Butzer and H. Berens, Semi-Groups of Operators and Approximation, SpringerVerlag. New York (1967).

[6] M. M. Cavalcanti, V. N. Domingos Cavalcanti and J. Ferreira, Existence and uniform decay for a non-linear viscoelastic equation with strong damping, Math. Methods. Appl. Sci. 24 (2001), 1043-1053.

[7] M. M. Cavalcanti, V. N. Domingos Cavalcanti, J. S. Prates Filho and J. A. Soriano, Existence and uniform decay rates for viscoelastic problems with nonlinear boundary damping, Differential and Integral equations 14 (2001), 85-116.

[8] M. M. Cavalcanti and H. Portillo Oquendo, Frictional versus viscoelastic damping in a semilinear wave equation, SIAM J. Control Optim. 42 (2003), 1310-1324.

[9] I. Chueshov and I. Lasiecka, Long-time behavior of second order evolution equation with nonlinear damping, Mem. Amer. Math. Soc. 195 (2008), AMS, Providence.

[10] I. Chueshov and I. Lasiecka, Von Karman Evolution Equations: Well-Posedness and Long-Time Dynamics, Springer Monographs in Mathematics (2010). 
[11] M. Conti, E. Marchini and V. Pata, A well posedness result for nonlinear viscoelastic equations with memory, Nonlinear Anal. 94 (2014), 206-216.

[12] C. M. Dafermos, Asymptotic stability in viscoelasticity, Arch. Rational Mech. Anal. 37 (1970), 297-308.

[13] M. Fabrizio and A. Morro, Mathematical problems in linear viscoelasticity, SIAM Studies in Applied Mathematics 12 (1992).

[14] G. B. Folland, Real Analysis: Modern Techniques and Their Applications, 2nd edition, Jhon Wiley \& Sons. Canada (1999).

[15] C. Giorgi, M. Grasseli and V. Pata, Well-posedness and longtime behavior of the phasefield model with memory in a history space setting, Quart. Appl. Math. 59 (2001), 701-736.

[16] J. K. Hale, Asymptotic Behavior of Dissipative Systems, Mathematical Surveys and Monographs, 25, American Mathematical Society, Providence (1988).

[17] X. Han and M. Wang, General decay of energy for a viscoelastic equation with nonlinear damping, Math. Methods Appl. 70 (2009), 346-358.

[18] X. Han and M. Wang, Global existence and uniform decay for a nonlinear viscoelastic equation with damping, Nonlinear Anal. 70 (2009), 3090-3098.

[19] J.-L. Lions, Quelques Méthodes de Résolution des Problèmes aux Limites Non Linéaires, Dunod Gauthier-Villars, Paris (1969).

[20] W. Liu, Uniform decay of solutions for a quasilinear system of viscoelastic equations, Nonlinear Anal. 71 (2009), 2257-2267.

[21] W. Liu, General decay and blow-up of solution for a quasilinear viscoelastic problem with nonlinear source, Nonlinear Anal. 73 (2010), 1890-1904.

[22] A. E. H. Love, Treatise on Mathematical Theory of Elasticity, Dover Publications (1944).

[23] T. F. Ma and P. N. Seminario Huertas, Attractors for a class of nonlinear viscoelastic equations with memory. (em preparação).

[24] S. A. Messaoudi, General decay of solutions of a viscoelastic equation, J. Math. Anal. Appl., 341 (2008), 1457-1467. 
[25] S. A. Messaoudi and N. Tatar, Global existence and uniform stability of solutions for a quasilinear viscoelastic problem, Math. Meth. Appl. Sci. 30 (2007), 665-680.

[26] S. A. Messaoudi and N. Tatar, Exponential and polynomial decay for a quasilinear viscoelastic equation, Nonlinear Anal. 68 (2008), 785-793.

[27] S. A. Messaoudi and N. Tatar, Exponential decay for a quasilinear viscoelastic equation, Math. Nachr. 282 (2009), 1443-1450.

[28] A. Miranville and S. Zelik, Handbook of Gifferential Equations, Handbook of Differential Equations, Evolutionary Equations, Volume 4, Chapter 3 (2008).

[29] J. E. Muñoz Rivera, Asymptotic behaviour in linear viscoelasticity, Quart. Appl. Math., 52 (1994), 628-648.

[30] V. Pata and A. Zucchi, Attractors for a damped hyperbolic equation with linear memory, Adv. Math. Sci. Appl., 11 (2001), 505-529.

[31] A. Pazy, Semigroups of Linear Operators and Applications to Partial Differential Equations, Applied Mathematical Sciences Vol. 44, Springer-Verlag, New York (1983).

[32] Y. Qin, J. Zhang and L. Sun, Upper semicontinuity of pullback attractors for a nonautonomous viscoelastic equation, Appl. Math. Comput. 223 (2013), 362-376.

[33] Y. Qin, B. Feng and M. Zhang, Uniform attractors for a non-autonomous viscoelastic equation with a past history, Nonl. Analysis 101 (2014) 1-15.

[34] J. Simon, Compact sets in the space $L^{p}(0, T ; B)$, Ann. Mat. Pura Appl. 146 (1987), 65-96.

[35] C. Sun, D. Cao and J. Duan, Non-autonomous wave dynamics with memory asymptotic regularity and uniform attractor, Discrete Contin. Dyn. Syst. Ser. B9 (2008), 743-761.

[36] R. Temam, Infinite-Dimensional Dynamical Systems in Mechanics and Physics, Appl. Math. Sci. 68 (1998).

[37] S. T. Wu, Exponential decay for a nonlinear viscoelastic equation with singular kernels, Acta Math. Sci. Ser. B. Engl. Ed. 32 (2012), 2237-2246. 
[38] S. T. Wu, General decay of solutions for a nonlinear system of viscoelastic wave equations with degenerate damping and source terms, J. Math. Anal. Appl. 406 (2013), $34-48$.

[39] K. Yosida, Functional Analysis, Springer-Verlag. Berlin (1965).

[40] E. Zeidler, Nonlinear Functional Analysis and its Applications. Part II-B, SpringerVerlag. New York (1990). 